\title{
STRING CONE AND SUPERPOTENTIAL COMBINATORICS FOR FLAG AND SCHUBERT VARIETIES IN TYPE A
}

\author{
L. BOSSINGER AND G. FOURIER
}

\begin{abstract}
We study the combinatorics of pseudoline arrangements and their relation to the geometry of flag and Schubert varieties. We associate to each pseudoline arrangement two polyhedral cones, defined in a dual manner. We prove that one of them is the weighted string cone by Littelmann and Berenstein-Zelevinsky. For the other we show how it arises in the framework of cluster varieties and mirror symmetry by Gross-Hacking-Keel-Kontsevich: for the flag variety the cone is the tropicalization of their superpotential while for Schubert varieties a restriction of the superpotential is necessary.

We prove that the two cones are unimodularly equivalent. As a corollary of our combinatorial result we realize Caldero's toric degenerations of Schubert varieties as GHKKdegeneration using cluster theory.
\end{abstract}

\section{INTRODUCTION}

Toric degenerations of Grassmannians (and later of flag and Schubert varieties) have gained much attention from the first works of Hodge et al, realized by Gonciulea and Lakshmibai [GL96]. Today it is a fast growing subject with strong links to representation theory, symplectic geometry, differential geometry, algebraic geometry. In the past twenty something years several approaches to construct toric degenerations have been provided: representation theory using dual canonical bases [Cal02], or more general using birational sequences [FFL17], Newton-Okounkov bodies using valuations [And13, KK12, Kav15], tropical geometry using Gröbner theory [MS15, KM16], and last but not least, cluster varieties and superpotentials [GHKK18, RW17].

We briefly recall two of these approaches in more detail focusing on the flag variety of full flags of subspaces in $\mathbb{C}^{n}$.

1.1. String cones. Littelmann in [Lit98] and Berenstein-Zelevinsky in [BZ01] introduced string cones to parametrize Lusztig's dual canonical basis of the quantum group. The defining hyperplanes of the cones are described inductively by Littelmann and explicitly by GleizerPostnikov [GP00] using pseudoline arrangements (see Definition 5). In these arrangements, associated to a fixed reduced expression of the longest Weyl group element, Gleizer and Postnikov describe the defining inequalities using the turning points of GP-paths (see Definition 8). This can be naturally generalized to Schubert varieties as done by Littelmann and generalized in Theorem 5 below.

We consider the flag variety as $S L_{n} / B$ and fix an embedding into projective space associated to a highest weight representation $V(\lambda)$ of $\mathfrak{s l}_{n}$. Then the string cones also parametrize a basis of the homogeneous coordinate ring of the flag variety. In this setup, Caldero used the string parametrization and the approach via Rees algebras to define toric degenerations of flag and Schubert varieties for reduced expressions of corresponding Weyl group elements in [Cal02]. The polytope associated to the toric fiber of the degeneration is the string polytope.

The first author was supported by "Programa de Becas Posdoctorales en la UNAM 2018" Instituto de Matemáticas, Universidad Nacional Autónoma de México. 
1.2. Cluster algebras. Cluster algebras were introduced in [FZ02] by Fomin and Zelevinsky and quickly grew to become a research area on their own. They are commutative rings endowed with seeds (maximal sets of algebraically independent generators) related by mutation (local transformations exchanging one seed by another). At their origin they are closely related to the representation theory of finite dimensional algebras, but also many objects related to algebraic groups have a cluster structure. For example, the homogeneous coordinate ring of Grassmannians (see [FZ02, Sco06]), double Bruhat cells (see [BFZ05]), (partial) flag varieties (see [GLS13]) or Richardson varieties (see [Lec16]).

A geometric approach to cluster algebras was introduced by Fock and Goncharov in [FG06]. In this setting they work with cluster varieties, schemes glued from algebraic tori (one for every seed) with gluing given by the birational transformations induced by mutation. They come in two flavours, $\mathcal{A}$ - and $\mathcal{X}$-cluster varieties, one being the mirror dual to the other as developed by Gross, Hacking, Keel and Kontsevich in [GHKK18]. Among other things, they define $\vartheta$-bases for cluster algebras and provide toric degenerations of (partial compactifications of) cluster varieties. The $\mathcal{X}$-cluster variety comes endowed with a Laurent polynomial, the superpotential, whose tropicalization gives a polyhedral cone and a polytope as a slice of this cone. The superpotential polytope is the polytope associated to the special fibre of the toric degeneration.

Question 1. Can Caldero's toric degenerations of flag and Schubert varieties be recovered by tropicalizing a superpotential?

A first hint towards a positive answer to this question was given by Magee in [Mag15]. He recovers the Gelfand-Tsetlin polytope ${ }^{1}$ as a superpotential polytope in a particular seed. Further results in this direction are obtained by Genz-Koshevoy-Schumann in [GKS16] and [GKS17], who generalize Magee's result to flag varieties of simple, simply connected, simply laced algebraic groups. They recover the classical string and Lusztig parametrizations from the superpotential.

1.3. Summary of the results. In [GP00] Gleizer and Postnikov use pseudoline arrangements associated to reduced expressions $\underline{w}_{0}$ of $w_{0} \in S_{n}$ and GP-paths in these to parametrize the inequalities for string cones $C_{\underline{w}_{0}} \subset \mathbb{R}^{N}$. We extend their result by adding weight inequalities encoded combinatorially in the pseudoline arrangement and obtain the weighted string cones $\mathcal{C}_{\underline{w}_{0}} \subset \mathbb{R}^{N+n-1}$ as defined in [Lit98]. Intersecting $\mathcal{C}_{\underline{w}_{0}}$ with the preimage of a weight $\lambda \in \mathbb{R}^{n-1}$ of an appropriate projection $\pi: \mathbb{R}^{N+n-1} \rightarrow \mathbb{R}^{n-1}$ yields the string polytope $\pi^{-1}(\lambda) \cap \mathcal{C}_{\underline{w}_{0}}$. Generalizing to arbitrary $w \in S_{n}$ and following Caldero [Cal02] we obtain similarly the string cone, weighted string cone and string polytope for the Schubert variety $X(w)$.

We introduce a second polyhedral cone $S_{\underline{w}_{0}} \subset \mathbb{R}^{N}$ associated to a pseudoline arrangement in a dual way: the variables are indexed by the faces of the diagram as opposed to the vertices in case of the string cone. From additional weight inequalities we get a weighted cone $\mathcal{S}_{\underline{w}_{0}}$ and from a second projection $\tau: \mathbb{R}^{N+n-1} \rightarrow \mathbb{R}^{n-1}$ we obtain polytopes $\tau^{-1}(\lambda) \cap \mathcal{S}_{\underline{w}_{0}}$ for $\lambda \in \mathbb{R}^{n-1}$. As in the case of string cones we provide these also for arbitrary $w \in S_{n}$.

The combinatorial result of our study is the following (see Theorem 4).

Theorem 1. For every $\underline{w} \in S_{n}$, the two cones $\mathcal{C}_{\underline{w}}$ and $\mathcal{S}_{\underline{w}}$ are unimodularly equivalent and the lattice-preserving linear map is given by the duality of faces and vertices in the pseudoline arrangement. Moreover, this linear map restricts to linear bijections between the polytopes $\pi^{-1}(\lambda) \cap \mathcal{C}_{\underline{w}} \cong \tau^{-1}(\lambda) \cap \mathcal{S}_{\underline{w}}$ and the cones $S_{\underline{w}} \cong C_{\underline{w}}$.

\footnotetext{
${ }^{1}$ Littelmann showed in [Lit98] that the Gelfand-Tsetlin polytope is unimodular equivalent to a certain string polytope.
} 
The cone $\mathcal{S}_{\underline{w}_{0}}$ appears in the framework of mirror symmetry for cluster varieties [GHKK18]: denote by $B^{-} \subset S L_{n}$ the Borel subgroup of lower triangular matrices and by $U \subset B$ (resp. $U^{-} \subset B^{-}$) the unipotent radical with all diagonal entries being 1. The double Bruhat cell $G^{e, w_{0}}=B^{-} \cap B w_{0} B$ is an $\mathcal{A}$-cluster variety (see [BFZ05]) and can be identified with an open subset of $B w_{0} B / U$. Let $\mathcal{X}$ the be dual of the $\mathcal{A}$-cluster variety $G^{e, w_{0}}$ and let $s_{0}=s_{\hat{w}_{0}}$ be the seed of the cluster algebra $\mathbb{C}\left[G^{e, w_{0}}\right]$ corresponding to the reduced expression $\underline{\hat{w}}_{0}=$ $s_{1} s_{2} s_{1} \cdots s_{n-1} \cdots s_{2} s_{1}$. Let $W$ be the superpotential defined by the sum of the $\vartheta$-functions for frozen variables in $s_{0}$ as introduced in [GHKK18]. Then $W^{\text {trop }}$ denotes the tropicalization of the superpotential. Magee has shown in [Mag15] (see also Goncharov-Shen in [GS15]) that

$$
\mathcal{S}_{\hat{\underline{\omega}}_{0}}=\left\{x \in \mathbb{R}^{N+n-1} \mid W^{\text {trop }}{\mid \mathcal{X}_{s_{0}}}(x) \geq 0\right\}=: \Xi_{s_{0}} \text {. }
$$

We show that mutation of the pseudoline arrangement and hence of the cone $\mathcal{S}_{\underline{w}_{0}}$, is compatible with mutation of the superpotential [GHK15] by introducing mutation of GP-paths. We obtain the following result ${ }^{2}$ (see Corollary 2):

Theorem 2. Let $\underline{w}_{0}$ be an arbitrary reduced expression of $w_{0} \in S_{n}$ and $s_{\underline{w}_{0}}$ be the seed corresponding to the pseudoline arrangement, $\mathcal{X}_{\underline{\underline{w}}_{0}}$ the toric chart of the seed $\underline{s}_{\underline{w}_{0}}$. Then

$$
\mathcal{S}_{\underline{w}_{0}}=\Xi_{{\underline{w_{0}}}_{0}},
$$

the polyhedral cone defined by the tropicalization of $W$ expressed in the seed $s_{\underline{w}_{0}}$.

Consider $w \in S_{n}$ arbitrary and $\underline{w}$ a reduced expression of $w$. Let $W$ be as above and consider its restriction $\operatorname{res}_{\underline{w}}\left(\left.W\right|_{\mathcal{X}_{\underline{s}_{0}}}\right)$ to the mirror dual of the $\mathcal{A}$-cluster variety $G^{e, w}$. Let $s_{\underline{w}}$ be the corresponding seed in the cluster algebra. Then the tropicalization of the restriction yields again a cone $\Xi_{s_{\underline{w}}}$. The last result establishes an answer to the question above for Schubert varieties (see Theorem 8 ).

Theorem 3. Let $\underline{w} \in S_{n}$, and fix $\underline{w}_{0}=\underline{w} s_{i_{\ell(w)+1}} \ldots s_{i_{N}}$ a reduced expression of $w_{0} \in S_{n}$. Let $s_{\underline{w}}$ resp. $s_{\underline{w}_{0}}$ be the corresponding seeds, then

$$
\mathcal{S}_{\underline{w}}=\Xi_{S_{\underline{w}}} .
$$

The article is structured as follows: after introducing relevant notation, we recall pseudoline arrangements and define the two collections of polyhedral objects and unimodular equivalences among them in $\S 3$. In $\S 4$ we show that one of the cones is the weighted string cone and in $\S 5$ we show how the other arises from the superpotential. Then in $\S 6$ we apply our combinatorial result and relate to toric degenerations.

Acknowledgements. Both authors would like to thank X. Fang for the stimulating and helpful discussions. In particular, we would like to thank T. Magee for explaining his results over and over again. We are grateful to P. Littelmann and A. Berenstein for inspiring conversations.

\section{Preparation}

2.1. Notation for $S L_{n}(\mathbb{C})$. We fix as Borel subgroup the upper triangular matrices $B \subset S L_{n}$ and diagonal matrices as maximal torus $T \subset B$. We denote the Borel subgroup of lower triangular matrices $B^{-}$(it is also called the opposite Borel subgroup of $B$ ). Inside of $B$ (resp. $B^{-}$) we have the subgroup of unipotent matrices $U$ (resp. $U^{-}$) with all diagonal entries being 1. They are the unipotent radical of $B$ (resp. $B^{-}$). Consider the Lie algebra $\operatorname{Lie}\left(S L_{n}\right)=\mathfrak{s l}_{n}$ and fix the Cartan decomposition $\mathfrak{s l}_{n}=\mathfrak{n}^{-} \oplus \mathfrak{h} \oplus \mathfrak{n}^{+}$. Let us denote the root system of $S L_{n}$

\footnotetext{
${ }^{2}$ Genz-Koshevoy-Schumann [GKS16] obtain a similar result in the context of crystal graphs.
} 
by $R \subset \mathbb{R}^{n}$. Denoting the standard basis of $\mathbb{R}^{n}$ by $\left\{\epsilon_{i}\right\}_{i=1, \ldots, n}$ we fix the the simple roots of $R$ to be $\alpha_{i}=\epsilon_{i}-\epsilon_{i+1}$ for $i=1, \ldots, n-1$. They generate the root lattice. The positive roots are denoted $R^{+}=\{\beta \in R \mid \beta>0\}$. They are of form $\alpha_{i, j}:=\alpha_{i}+\cdots+\alpha_{j}$ for $i \leq j<n$. With our choice of simple roots we have $\alpha_{i, j}=\epsilon_{i}-\epsilon_{j+1}$. The number of positive roots is denoted by $N=\frac{n(n-1)}{2}$. For a positive $\operatorname{root} \beta=\alpha_{i, j}$ let $f_{\beta} \in \mathfrak{n}^{-}$be the root vector of weight $-\beta$. For the weight lattice we choose the notation $\Lambda$ with generators the fundamental weights being $\omega_{1}, \ldots, \omega_{n-1}$. Let $\Lambda^{+}$denote the dominant integral weights in $\Lambda$. By $\Lambda^{++}$we denote the set of regular dominant weights, i.e. those $\lambda=\sum_{i=1}^{n-1} a_{i} \omega_{i}$ with $a_{i} \in \mathbb{Z}_{>0}$. For every $\lambda \in \Lambda^{+}$there is a (finite-dimensional) irreducible representation of $\mathfrak{s l}_{n}$ of highest weight $\lambda$, denote it by $V(\lambda)$. It is cyclically generated by a highest weight vector $v_{\lambda} \in V(\lambda)$ (unique up to scaling) over $U\left(\mathfrak{n}^{-}\right.$), the universal enveloping algebra of $\mathfrak{n}^{-}$. The Weyl group of $S L_{n}$ is the symmetric group $S_{n}$ generated by the simple transpositions $s_{i}=(i, i+1)$ for $1 \leq i<n$. By $w_{0}$ we denote the longest element in $S_{n}$. For every $w \in S_{n}$, we denote by $\ell(w)$ the minimal length of $w$ as a word in the generators $s_{i}$. Further, $\underline{w}$ denotes a reduced expression $\underline{w}=s_{i_{1}} \cdots s_{i_{\ell(w)}}$. Such an expression is not unique. For any two reduced expressions of $w$ there is a sequence of local transformations leading from one to the other. These local transformations are either swapping orthogonal reflections $s_{i} s_{j}=s_{j} s_{i}$ if $|i-j|>1$ or exchanging consecutive $s_{i} s_{i+1} s_{i}=s_{i+1} s_{i} s_{i+1}$. The symmetric group acts on the weight lattice. Fix $w \in S_{n}$ and $\lambda \in \Lambda^{+}$, then the weight space of weight $w(\lambda)$ in $V(\lambda)$, denoted $V(\lambda)_{w(\lambda)}$, is called extremal and it is one-dimensional.

Definition 1. For $w \in S_{n}$ and $\lambda \in \Lambda^{+}$we fix $v_{w \lambda} \in V(\lambda)_{w \lambda}$ we consider $U(\mathfrak{b}) \cdot v_{w \lambda}=: V_{w}(\lambda)$. This is a $\mathfrak{b}$-module called the Demazure module.

Note that though $V_{w}(\lambda)$ is a $\mathfrak{b}$-submodule of $V(\lambda)$, it is not an $\mathfrak{s l}_{n}$-module. For a Demazure module $V_{w}(\lambda)$ we denote by $V_{w}(\lambda)^{\perp}$ its orthogonal complement in $V(\lambda)^{*}$.

For $\lambda \in \Lambda^{+}$let $L_{\lambda}$ be the total space of the homogeneous line bundle associated to the weight $\lambda$ over $S L_{n} / B$. These line bundles satisfy $L_{m \lambda}=L_{\lambda}^{\otimes m}$ for $m \geq 1$ and are ample, if $\lambda \in \Lambda^{++}$. By the Borel-Weil-Theorem we have

$$
H^{0}\left(S L_{n} / B, L_{\lambda}\right)^{*} \cong V(\lambda)
$$

This correspondence induces an embedding $S L_{n} / B \hookrightarrow \mathbb{P}(V(\lambda))$, by $g B \mapsto g\left[v_{\lambda}\right]$. In particular, we can realize the homogeneous coordinate ring of the flag variety as $\mathbb{C}\left[S L_{n} / B\right]=$ $\bigoplus_{k>0} V(k \lambda)^{*}$. Similarly, we obtain $\mathbb{C}\left[S L_{n} / U\right]=\bigoplus_{\lambda \in \Lambda^{+}} V(\lambda)$ which is a consequence of the Peter-Weyl-Theorem. The quasi-affine variety $S L_{n} / U$ is also called base affine space.

We consider for $w \in S_{n}$ the Bruhat cell $B w B \subset S L_{n}$. The quotient $B w B / B$ is called $S c h u$ bert cell and its Zariski closure $X_{w}:=\overline{B w B / B} \subset S L_{n} / B$ is the Schubert variety. Schubert varieties are normal, not necessarily smooth (but if singular having only rational singularities) subvarieties of the flag variety. Their dimension equals the length of the associated Weyl group element, i.e. $\operatorname{dim} X_{w}=\ell(w)$. The line bundles $L_{\lambda}$ can be restricted to Schubert varieties and the Borel-Weil Theorem generalizes as follows. Fix $w \in S_{n}$ and $\lambda \in \Lambda^{+}$, then

$$
H^{0}\left(X_{w}, L_{\lambda}\right)^{*} \cong V_{w}(\lambda) \text {. }
$$

2.2. Cluster algebras. For a reminder on cluster algebras we refer the reader to [FZ02] and [BFZ05]. We review below $\mathcal{A}$ - and $\mathcal{X}$-cluster varieties as introduced in [FG06], following $[$ GHK15, §2]. As the cluster algebra we are interested in is skew-symmetric and of geometric type we restrict to this case.

Definition 2. The skew-symmetric fixed data $\Gamma$ consists of

- a finite set $I=\{1, \ldots, m\}$ of directions with a subset of unfrozen directions $I_{\mathrm{uf}}=$ $\{1, \ldots, n\}$ 
- a lattice $N$ of $\operatorname{rank}|I|$;

- a saturated sublattice $N_{\mathrm{uf}} \subseteq N$ of rank $\left|I_{\mathrm{uf}}\right|$;

- a skew-symmetric bilinear form $\{\cdot, \cdot\}: N \times N \rightarrow \mathbb{Q}$;

- a sublattice $N^{\circ} \subseteq N$ of finite index satisfying

$$
\left\{N_{\mathrm{uf}}, N^{\circ}\right\} \subset \mathbb{Z} \text { and }\left\{N, N_{\mathrm{uf}} \cap N^{\circ}\right\} \subset \mathbb{Z}
$$

- $M=\operatorname{Hom}(N, \mathbb{Z})$ and $M^{\circ}=\operatorname{Hom}\left(N^{\circ}, \mathbb{Z}\right)$.

A seed $s$ is a basis $\left\{e_{1, s}, \ldots, e_{m, s}\right\}$ of $N=\mathbb{Z}^{m}$. We sometimes write $N_{s}$ to refer to $N$ with the associated basis. For a fixed seed $s$ the bilinear form induces a quiver $Q_{s}$ given by its exchange matrix $\varepsilon_{s}:=\left(\left\{e_{i, s}, e_{j, s}\right\}\right)_{i j}$. Let $\left\{f_{1, s}, \ldots, f_{m, s}\right\}$ be the dual basis for $M$. To each seed we associate two tori $T_{N} \cong\left(\mathbb{C}^{*}\right)^{m} \cong T_{M}$ by

$$
\mathcal{X}_{s}:=T_{M, s}=\operatorname{Spec}(\mathbb{C}[N]) \text { and } \mathcal{A}_{s}:=T_{N, s}=\operatorname{Spec}(\mathbb{C}[M]) .
$$

We denote the coordinates on $\mathcal{X}_{s}$ by $X_{1, s}, \ldots, X_{m, s}$. Corresponding to the basis of the lattice we have $X_{i, s}:=z^{e_{i, s}}$. When the seed we are working in is clear we drop it from the notation. We define mutation at $k \in I_{\mathrm{uf}}$ on the basis $\left\{e_{i, s}\right\}$ of the lattice $N$ for seed $s$ by

$$
e_{i, s^{\prime}}:=\left\{\begin{array}{cc}
e_{i, s}+\max \left\{\epsilon_{i k}, 0\right\} e_{k, s}, & \text { for } i \neq k, \\
-e_{k, s}, & \text { for } i=k .
\end{array}\right.
$$

Then $\left\{e_{1, s^{\prime}}, \ldots, e_{m, s^{\prime}}\right\}$ forms again a basis for $N$ associated with the seed $s^{\prime}=\mu_{k}(s)$. The dual basis for $M$ transforms as

$$
f_{i, s^{\prime}}:=\left\{\begin{array}{cc}
-f_{i, s}, & \text { for } i \neq k, \\
f_{k, s}+\sum_{j} \max \left\{-\epsilon_{k j}, 0\right\} f_{j, s}, & \text { for } i=k .
\end{array}\right.
$$

Then $\left\{f_{1, s^{\prime}}, \ldots, f_{m, s^{\prime}}\right\}$ is the dual basis for $M$ associated with $s^{\prime}=\mu_{k}(s)$. Mutation induces birational maps between the tori

$$
\mu_{k}: \mathcal{X}_{s} \rightarrow \mathcal{X}_{\mu_{k}(s)} \text { and } \mu_{k}: \mathcal{A}_{s} \rightarrow \mathcal{A}_{\mu_{k}(s)}
$$

defined by the pullback of functions. We have for $\mathcal{X}$-tori

$$
\mu_{k}^{*}\left(z^{n}\right):=z^{n}\left(1+z^{e_{k, s}}\right)^{-\left\{n, e_{k, s}\right\}_{s}}, \text { for } n \in N .
$$

For the $\mathcal{A}$-tori the birational map is induced by

$$
\mu_{k}^{*}\left(A_{k, s^{\prime}}\right)=\left\{\begin{array}{cc}
A_{i, s}, & \text { for } i \neq k, \\
\frac{\prod_{i \rightarrow k \in Q_{s}} A_{i, s}+\prod_{k \rightarrow j \in Q_{s}} A_{j, s}}{A_{k, s}}, & \text { for } i=k .
\end{array}\right.
$$

Note that the variables $A_{i, s}$ for $n+1 \leq i \leq m$ never change under mutation, we can therefore drop the $s$ from their index. To be consistent with the $\mathcal{X}$-notation, we set $A_{i, s}=z^{f_{i, s}}$. We denote by $s_{0}$ a fixed initial seed for $\Gamma$ and for any other seed obtained from $s_{0}$ by a sequence of mutations we write $s \sim s_{0}$. By [GHK15, Proposition 2.4] we can give the following definition (see [BFMN18, §3] for a more detailed description).

Definition 3. Given fixed data and a fixed initial seed $s_{0}$ the $\mathcal{X}$ - (resp. $\mathcal{A}$-) cluster variety is defined as the scheme

$$
\left.\mathcal{X}:=\bigcup_{s \sim s_{0}} \mathcal{X}_{s} \text { (resp. } \mathcal{A}:=\bigcup_{s \sim s_{0}} \mathcal{A}_{s}\right)
$$

obtained by gluing the tori $\mathcal{X}_{s}\left(\right.$ resp. $\mathcal{A}_{s}$ ) along the birational maps induced by mutation. 
The relation to cluster algebras is the following. The global sections of the structure sheaf on $\mathcal{A}$ are related to the upper cluster algebra $\overline{\mathcal{Y}}\left(s_{0}\right)$ (see [BFZ05, Definition 1.1]) associated to the initial data with initial seed $s_{0}$ :

$$
H^{0}(\mathcal{A}, \mathcal{O}(\mathcal{A}))=\overline{\mathcal{Y}}\left(s_{0}\right) \otimes_{\mathbb{Z}} \mathbb{C}
$$

A natural (partial) compactification $\overline{\mathcal{A}}$ of $\mathcal{A}$ (an $\mathcal{A}$-cluster variety) is given by allowing the frozen variables $A_{n+1}, \ldots, A_{m}$ to vanish. We denote the resulting boundary divisor in $\overline{\mathcal{A}}$ by

$$
D:=\sum_{f=n+1}^{m} D_{f}, \text { where } D_{f}:=\left\{A_{f}=0\right\} \subset \overline{\mathcal{A}} \text {. }
$$

Every component $D_{f}$ of the boundary divisor induces a (rank 1 ) valuation $\operatorname{ord}_{D_{f}}: \mathbb{C}[\mathcal{A}] \rightarrow \mathbb{Z}$ by sending a function $g \in \mathbb{C}[\mathcal{A}]$ to its order of vanishing along $D_{f}$. If $g$ has a pole along $D_{f}$, then $\operatorname{ord}_{D_{f}}(g)<0$ is the order of the pole. These valuations are called divisorial discrete valuations in [GHKK18].

A main result of [GHKK18] is the definition and parametrization of the $\vartheta$-basis for $\mathbb{C}[\mathcal{A}]$. One central question is: When is a basis element of $\mathbb{C}[\mathcal{A}]$ also a basis element for $\mathbb{C}[\overline{\mathcal{A}}]$ ?

The full Fock-Goncharov conjecture (see [GHKK18, Definition 0.6]) suggests that basis elements for $\mathbb{C}[\mathcal{A}]$ are parametrized by tropical points in $\mathcal{X}^{\text {trop }}(\mathbb{Z})$ (see $[\mathrm{GHKK} 18, \S 2]$ ). We will not go into detail about this tropical space due to the following fact: fixing a seed $s$ we have an isomorphism

$$
\left.\mathcal{X}^{\operatorname{trop}}(\mathbb{Z})\right|_{s} \cong N_{s} \cong \mathbb{Z}^{m}
$$

For the purpose of this paper we always work in a fixed seed and therefore have an identification of lattice points in $N_{s}$ with basis elements for $\mathbb{C}[\mathcal{A}]$. From now on we assume that the cluster variety $\mathcal{A}$ satisfies the full FG-conjecture, as this is the case for the cluster variety we are interested in: Magee showed in [Mag15] that the full FG-conjecture is satisfied by the cluster variety inside $S L_{n} / U$. A number of criteria for the full Fock-Goncharov conjecture to hold are discussed in [GHKK18, §8.4] and we refer the interested reader there for more details.

Associated to each component of the boundary divisor there exists a function $\vartheta_{f}$ on the dual cluster variety $\mathcal{X}$. Assuming the full FG-conjecture we can compute and expression for $\vartheta_{f}$ in $\mathcal{X}_{s_{0}}\left(s_{0}\right.$ being a fixed initial seed $)$ as described by the Algorithm 1.

Definition 4. Let $\mathcal{A}$ be an $\mathcal{A}$-cluster variety satisfying the full Fock-Goncharov conjecture. Then we define the superpotential $W: \mathcal{X} \rightarrow \mathbb{C}$ on the dual cluster variety $\mathcal{X}$ as

$$
W:=\sum_{f \text { frozen vertex in } Q} \vartheta_{f} .
$$

Remark 1. A seed $s_{f}$ for which a frozen vertex $f$ is a sink (as in the first step of Algorithm 1) is called optimized for $f$. Finding an optimized seed for a frozen vertex is in general a hard problem as there might be infinitely many seeds. Further, doing these computations by hand is already after a few mutation quite frustrating due to the recursive formulas. An excellent tool for such computations is provided by Keller's quiver mutation applet [Kel].

Coming back to $\mathbb{C}[\mathcal{A}]$, note that a basis element $\vartheta \in \mathbb{C}[\mathcal{A}]$ gives an element in $\mathbb{C}[\overline{\mathcal{A}}]$ if $\operatorname{ord}_{D_{f}}(\vartheta) \geq 0$ for every component $D_{f}$ of the boundary divisor. In particular,

$$
\vartheta \in \mathbb{C}[\overline{\mathcal{A}}] \Leftrightarrow \min _{f \text { frozen }}\left\{\operatorname{ord}_{D_{f}}(\vartheta)\right\} \geq 0
$$

We need the following notion of tropicalization for Laurent polynomials. The tropicalization of $g=\sum_{\mathbf{u} \in \mathbb{Z}^{m}} a_{\mathbf{u}} z^{\mathbf{u}} \in \mathbb{C}\left[z_{1}^{ \pm 1}, \ldots, z_{m}^{ \pm 1}\right]$ is the function $g^{\text {trop }}: \mathbb{R}^{m} \rightarrow \mathbb{R}$ given by

$$
g^{\text {trop }}(\mathbf{x}):=\min \left\{\mathbf{x} \cdot \mathbf{u} \mid \mathbf{u} \in \mathbb{Z}^{m} \text { and } a_{\mathbf{u}} \neq 0\right\} .
$$




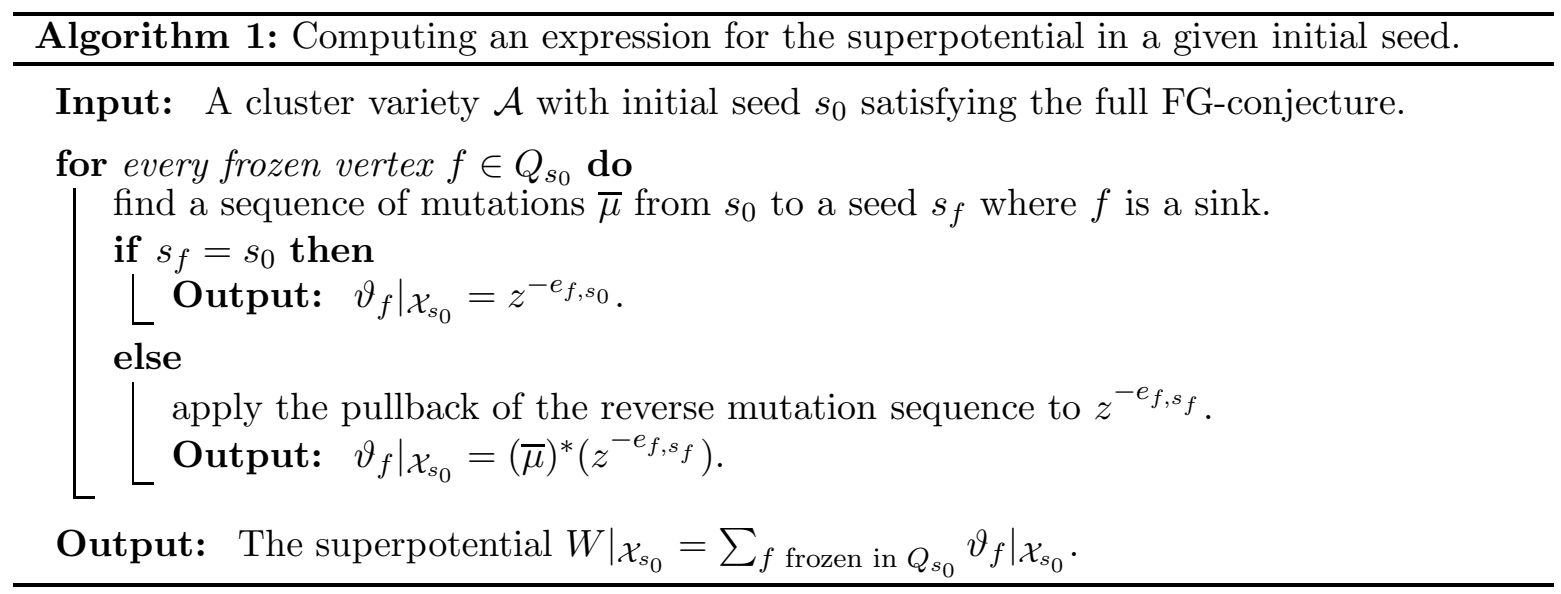

Let $g_{\vartheta} \in N_{s}$ be the lattice point associated to $\vartheta$ for a fixed seed $s$. Then using the fact that $\vartheta_{f}^{\text {trop }}\left(g_{\vartheta}\right)=\operatorname{ord}_{D_{f}}(\vartheta)$, this translates to

$$
\vartheta \in \mathbb{C}[\overline{\mathcal{A}}] \Leftrightarrow g_{\vartheta} \in\left\{\mathbf{x} \in \mathbb{R}^{m}|W|_{\mathcal{X}_{s}}^{\text {trop }}(\mathbf{x}) \geq 0\right\} \cap N_{s} .
$$

In particular, the lattice points in $\left\{\mathbf{x} \in \mathbb{R}^{m}|W|_{\mathcal{X}_{s}}^{\text {trop }}(\mathbf{x}) \geq 0\right\}$ parametrize a basis for $\mathbb{C}[\overline{\mathcal{A}}]$.

\section{Pseudoline arrangements and Gleizer-Postnikov paths}

In the following section we develop the combinatorial background and prove the combinatorial Theorem 1, whose main applications are Theorems $2 \& 3$ stated in the introduction. In particular, with Theorem 1 we Question 1, which is the aim of this article.

Recall our notation for the symmetric group $S_{n}$ from $\S 2.1$. We associate for each $w \in S_{n}$ a diagram called a pseudoline arrangement to every reduced expression $\underline{w}$. These diagrams turn out to be closely related to cluster algebras. In fact, to every pseudoline arrangement one can associate a quiver and then using the construction summarized in $\S 2.2$ define a cluster algebra. We start by introducing the combinatorial tools: to a pseudoline arrangement we associate two weighted cones and give a unimodular equivalence between them.

Definition 5. A pseudoline arrangement $\mathrm{pa}(\underline{w})$ associated to a reduced expression $\underline{w}=$ $s_{i_{1}} \cdots s_{i_{\ell(w)}}$ is a diagram consisting of $n$ horizontal pseudolines $l_{1}, \ldots, l_{n}$ (or short lines) labelled at the left end from bottom to top, with crossings indicated by the reduced expression. A reflection $s_{i}$ indicates a crossing at level $i$ (see e.g. Figure 4).

For a given reduced expression $\underline{w}=s_{i_{1}} \cdots s_{i_{l(w)}}$, we associate to each $s_{i_{j}}$ the positive root $\beta_{i_{j}}:=s_{i_{1}} \cdots s_{i_{j-1}}\left(\alpha_{i_{j}}\right)$. Then $\beta_{i_{j}}=\alpha_{k, m-1}$ for $k, m<n$ and $s_{i_{j}}$ induces the crossing of the lines $l_{k}$ and $l_{m}$ in $\mathrm{pa}(\underline{w})$. The crossing point is a vertex in the diagram and it is labelled $(k, m)$. As two lines $l_{k}, l_{m}$ cross at most once, there is at most one position with label $(k, m)$. For a given $w$ the pairs appearing as labels for crossing points are exactly those for which $w\left(\alpha_{k, m-1}\right)<0$. Further, the right end of a pseudoline $l_{i}$ is a vertex labelled $L_{i}$. Let $\mathrm{pa}(\underline{w})_{0}$ be the set of all vertices in $\mathrm{pa}(\underline{w})$.

Definition 6. [BFZ05, Definition 2.2] Let $w \in S_{n}$ with reduced expression $\underline{w}$. Then the quiver $Q_{\underline{w}}$ associated to $\mathrm{pa}(\underline{w})$ has vertices $w_{F}$ associated to faces $F$ of $\mathrm{pa}(\underline{w})$ and arrows:

(1) if two faces are at the same level separated by a crossing then there is an arrow from

left to right (see Figure 1a);

(2) if two faces are on consecutive levels separated by two crossings then there is an arrow 
from right to left (either upwards or downwards, see Figure 1b, 1c).

Vertices corresponding to unbounded faces are frozen and we disregard arrows between them. All the other vertices are called mutable.

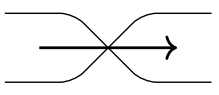

$a$

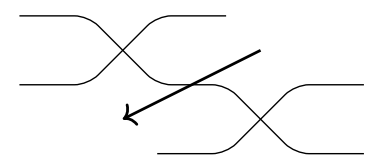

$b$

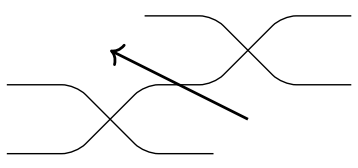

$c$

FiguRE 1. Arrows of the quiver arising from the pseudoline arrangement.

Definition 7. Let $w \in S_{n}$ with reduced expression $\underline{w}$. A mutation of pa $(\underline{w})$ (resp. of $\underline{w}$ ) is a change of consecutive $s_{r} s_{r+1} s_{r}$ in $\underline{w}$ to $s_{r+1} s_{r} s_{r+1}$ (or vice versa) (see Figure 2). We call a face $F$ of pa $(\underline{w})$ mutable if it corresponds to $s_{r} s_{r+1} s_{r}$ (or $s_{r+1} s_{r} s_{r+1}$ ) and denote the corresponding mutation by $\mu_{F}$. The resulting pseudoline arrangement is associated to the reduced expression $\mu_{F}(\underline{w})$ of $w$ and denoted by $\operatorname{pa}\left(\mu_{F}(\underline{w})\right)$.

Mutation of pseudoline arrangements is a special case quiver mutation (see e.g. [FWZ16, Definition 2.1.1])
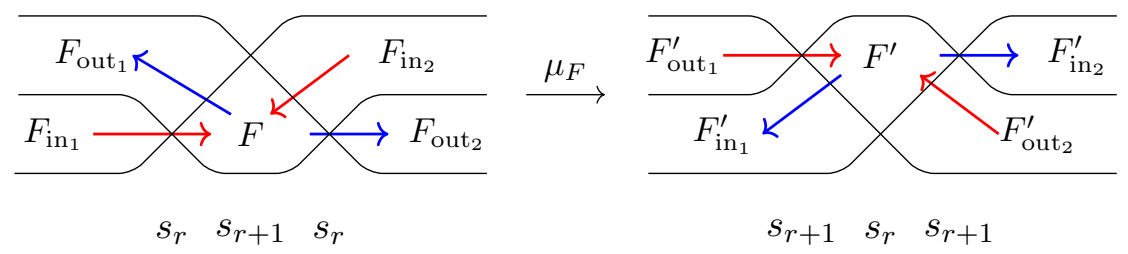

FiguRE 2. Mutation of pseudoline arrangements.

Note, that the quivers $Q_{\underline{w}}$ and $Q_{\mu_{F(\underline{w})}}$ are related by quiver mutation at the vertex $w_{F}$. However, $Q_{\underline{w}}$ has more mutable vertices than pa $(\underline{w})$ has mutable faces. When mutating $Q_{\underline{w}}$ at a vertex $w_{F^{\prime}}$ with $F^{\prime}$ not mutable in $\operatorname{pa}(\underline{w})$, then for $\mu_{F^{\prime}}\left(Q_{\underline{w}}\right)$ there is no reduced expression of $w$ that would give rise to this quiver via a pseudoline arrangement.

Consider $\underline{w}_{0} \in S_{n}$ with reduced expression $\underline{\hat{w}}_{0}:=s_{1} s_{2} s_{1} s_{3} s_{2} s_{1} \ldots s_{n-1} s_{n-2} \ldots s_{3} s_{2} s_{1}$ and the quiver $Q_{\underline{\hat{w}}_{0}}$. We label the vertices for faces $F_{(i, j)}$ bounded to the left by the crossing of lines $l_{i}$ and $l_{j}$ by $w_{(i, j)}$. In particular, the frozen vertices at the right boundary are labelled $w_{(n-1, n)}, \ldots, w_{(1, n)}$ from bottom to top. Referring to their level, the frozen vertices on the left boundary are labelled by $w_{1}, \ldots, w_{n-1}$ from bottom to top. In the following example we describe the quiver corresponding to this initial reduced expression $\underline{\hat{w}}_{0}$ for $n=5$.

Example 1. Consider $\underline{\hat{w}}_{0}=s_{1} s_{2} s_{1} s_{3} s_{2} s_{1} s_{4} s_{3} s_{2} s_{1} \in S_{5}$. The pseudoline arrangement and the corresponding quiver are depicted in Figure 3.

3.1. Orientation and paths. For every pair $\left(l_{i}, l_{i+1}\right)$ with $1 \leq i \leq n-1$ we give an orientation to a pseudoline arrangement by orienting lines $l_{1}, \ldots, l_{i}$ from right to left and lines $l_{i+1}, \ldots, l_{n}$ from left to right, see Figure 4 . Consider an oriented path with three consecutive crossings $v_{k-1} \rightarrow v_{k} \rightarrow v_{k+1}$ belonging to the same pseudoline $l_{i}$. Then $v_{k}$ is the intersection 


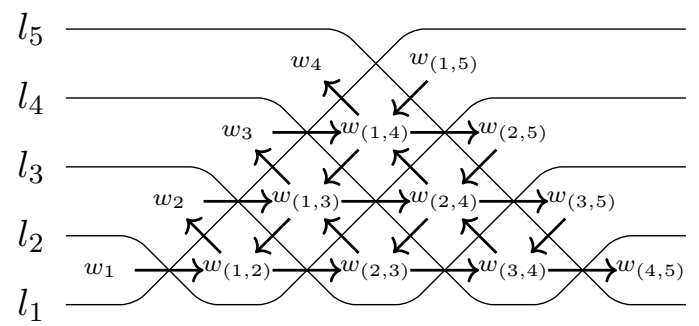

Figure 3. $\operatorname{pa}\left(\underline{\hat{w}}_{0}\right)$ and $Q_{\underline{\hat{w}}_{0}}$ with $\underline{\hat{w}}_{0}=s_{1} s_{2} s_{1} s_{3} s_{2} s_{1} s_{4} s_{3} s_{2} s_{1} \in S_{5}$.

of $l_{i}$ with some line $l_{j}$, i.e. $v_{k}=v_{(i, j)}$. If either $i<j$ and both lines are oriented to the left, or $i>j$ and both lines are oriented to the right, the path is called non-rigorous. Figure 5 shows these two situations. A path is called rigorous if it is not non-rigorous.

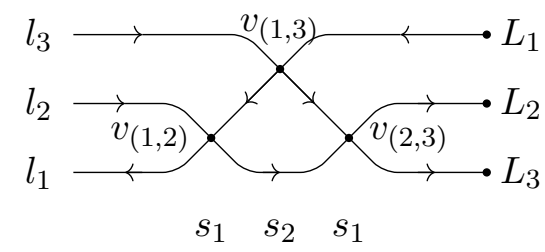

FiguRE 4. $\operatorname{pa}\left(\underline{w}_{0}\right)$ for $\underline{w}_{0}=s_{1} s_{2} s_{1} \in S_{3}$ with orientation for $\left(l_{1}, l_{2}\right)$.

Definition 8. Let $\underline{w}$ be a fixed reduced expression of $w \in S_{n}$. A Gleizer-Postnikov path (or short GP-path) is a rigorous path $\mathbf{p}$ in $\mathrm{pa}(\underline{w})$ endowed with some orientation $\left(l_{i}, l_{i+1}\right)$ for $i \in[n-1]:=\{1, \ldots, n-1\}$. It has source $L_{p}$ and $\operatorname{sink} L_{q}$ for $p \leq i$ and $q \geq i+1$. Further, $w(i+1) \leq w(p) \leq w(i)$ and $w(i+1) \leq w(q) \leq w(i)$. The set of all GP-paths for all orientations in the pseudoline arrangement associated to $\underline{w}$ is denoted by $\mathcal{P}_{\underline{w}}$.
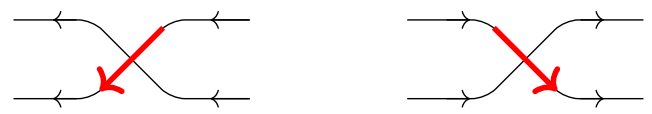

FiguRE 5. The two red arrows are forbidden in rigorous paths.

Note that if $w(i)<w(i+1)$ there are no GP-paths of shape $\left(l_{i}, l_{i+1}\right)$ and in case $w(p) \leq w(q)$ there are no GP-paths with source $L_{p}$ and sink $L_{q}$.

Proposition 1. Let $w \in S_{n}$ with reduced expression $\underline{w}$. Consider $\mathbf{p} \in \mathcal{P}_{\underline{w}}$ of shape $\left(l_{i}, l_{i+1}\right)$. Then $\mathbf{p}$ is either the empty path or does not cross the lines $l_{i+1}$ and $l_{i}$. In particular, $\mathbf{p}$ does not leave the area in $\mathrm{pa}(\underline{w})$ bounded by $l_{i}$ and $l_{i+1}$ to the left.

Proof. Without loss of generality we assume $w(i)<w(i+1)$, otherwise $\mathbf{p}$ is empty and we are done. Further, let $L_{p}$ be the source of $\mathbf{p}$ and $L_{q}$ the sink. We assume $w(p) \leq w(q)$, otherwise, again, $\mathbf{p}$ is empty. We focus on the part of $\mathrm{pa}(\underline{w})$ to the right of the crossing of $l_{i}$ and $l_{i+1}$ (which exists as $w(i)<w(i+1)$ ). Observe the following:

- all lines crossing $l_{i}$ do so oriented from top to bottom.

- all lines crossing $l_{i+1}$ do so oriented from bottom to top. 
As $L_{p}$ and $L_{q}$ lie in between the lines $l_{i}$ and $l_{i+1}$ this observation implies that $\mathbf{p}$ can not cross $l_{i}$ and if it was to cross $l_{i+1}$ it could not return to $L_{q}$, a contradiction. The only possibility that is left, is if $\mathbf{p}$ was to follow $l_{i}$ through the crossing with $l_{i+1}$, but then again, it could not return to $L_{q}$.

3.2. Cones and polytopes arising from pseudoline arrangements. We define two weighted cones, two cones, and two families of polytopes that arise from $\mathcal{P}_{\underline{w}}$ for $\underline{w}$ reduced expression of $w \in S_{n}$. We relate the two cones in the forthcoming sections, one to the weighted string cone (introduced by Littelmann [Lit98] and Berenstein-Zelevinsky [BZ01]), the other to the tropicalization of the (restriction of the) superpotential for a double Bruhat cell (see Magee [Mag15]).

3.3. The (weighted) GP-cone. For $\underline{w}=s_{i_{1}} \ldots s_{i_{\ell(w)}}$ we label the standard basis of $\mathbb{R}^{\ell(w)}$ by crossing points in $\mathrm{pa}(\underline{w})$, i.e. $\left\{c_{(k, m)} \mid w\left(\alpha_{k, m-1}\right)<0\right\}$. Sometimes it is convenient to use the notation $c_{i_{j}}:=c_{(k, m)}$, when $s_{i_{j}}$ induces the crossing of $l_{k}$ and $l_{m}$ in $\mathrm{pa}(\underline{w})$. Consider $\mathbf{p} \in \mathcal{P}_{\underline{w}}$. It is uniquely determined by those vertices in $\mathrm{pa}(\underline{w})_{0}$ where $\mathbf{p}$ changes from one line to another. For some $1 \leq p \leq i<q \leq n$ we can therefore write $\mathbf{p}$ as

$$
\mathbf{p}=L_{p} \rightarrow v_{\left(p, j_{1}\right)} \rightarrow v_{\left(j_{1}, j_{2}\right)} \rightarrow \cdots \rightarrow v_{\left(j_{k}, q\right)} \rightarrow L_{q}
$$

Set $j_{0}:=p$ and $j_{k+1}:=q$, then we associate to $\mathbf{p}$ the vector

$$
c_{\mathbf{p}}:=\sum_{s=0}^{k} c_{\left(j_{s}, j_{s+1}\right)} \in \mathbb{R}^{\ell(w)},
$$

where we set $c_{(i, j)}:=-c_{(j, i)}$ if $i>j$ and $c_{(i, i)}:=0$.

Definition 9. The following polyhedral cone is called GP-cone (due to Gleizer-Postnikov [GP00] who call it principal cone):

$$
C_{\underline{w}}=\left\{\mathbf{x} \in \mathbb{R}^{\ell(w)} \mid\left(c_{\mathbf{p}}\right)^{t}(\mathbf{x}) \geq 0, \forall \mathbf{p} \in \mathcal{P}_{\underline{w}}\right\} .
$$

Example 2. Consider the reduced expression $\underline{w}_{0}=s_{1} s_{2} s_{1} \in S_{3}$. We endow pa $\left(s_{1} s_{2} s_{1}\right)$ with the orientation for $\left(l_{1}, l_{2}\right)$, i.e. $l_{1}$ is oriented to the left and $l_{2}, l_{3}$ are oriented to the right (see Figure 4). There are two paths in $\mathcal{P}_{s_{1} s_{2} s_{1}}$ from $L_{1}$ to $L_{2}$,

$$
\mathbf{p}_{1}=L_{1} \rightarrow v_{(1,3)} \rightarrow v_{(1,2)} \rightarrow v_{(2,3)} \rightarrow L_{2} \text { and } \mathbf{p}_{2}=L_{1} \rightarrow v_{(1,3)} \rightarrow v_{(2,3)} \rightarrow L_{2} .
$$

They yield $c_{\mathbf{p}_{1}}=c_{(1,2)}$ and $c_{\mathbf{p}_{2}}=c_{(1,3)}-c_{(2,3)}$. Similarly for the orientation $\left(l_{2}, l_{3}\right)$ we find a path $\mathbf{p}_{3}=L_{2} \rightarrow v_{(2,3)} \rightarrow L_{3}$ with $c_{\mathbf{p}_{3}}=c_{(2,3)}$. Then

$$
C_{s_{1} s_{2} s_{1}}=\left\{\left(x_{(1,2)}, x_{(1,3)}, x_{(2,3)}\right) \in \mathbb{R}^{3} \mid x_{(1,2)} \geq 0, x_{(1,3)} \geq x_{(2,3)} \geq 0\right\} .
$$

We are interested in a weighted version of this cone to relate it to string polytopes in the next section. The weighted cone lives in $\mathbb{R}^{\ell(w)+n-1}$, where the additional basis elements are indexed $c_{1}, \ldots, c_{n-1}$. By some abuse of notation we denote by $c_{\mathbf{p}}$ also the vector $\left(c_{\mathbf{p}}, 0 \ldots, 0\right) \in$ $\mathbb{R}^{\ell(w)} \times\{0\}^{n-1} \subset \mathbb{R}^{\ell(w)+n-1}$.

For every $i \in[n-1]$ we define the following subset of $[\ell(w)]$

$$
J(i):=\left\{k \in[\ell(w)] \mid s_{i_{k}}=s_{i}\right\} \text { with } n_{i}:=\# J(i) .
$$

Let $J(i)=\left\{j_{1}, \ldots, j_{n_{i}}\right\}$, then we set $c_{[i: 0]}:=c_{i}$ and for $1 \leq k \leq n_{i}$ we define

$$
c_{[i: k]}:=c_{i}-c_{i_{j_{k}}}-2 \sum_{j \in J(i), j>j_{k}} c_{i_{j}}+\sum_{l \in J(i-1) \cup J(i+1), l>j_{k}} c_{i_{l}} .
$$

These vectors are normal vectors to the faces of the following weighted cone. 
Definition 10. The weighted Gleizer-Postnikov cone $\mathcal{C}_{\underline{w}} \subset \mathbb{R}^{\ell(w)+n-1}$ is defined as

$$
\mathcal{C}_{\underline{w}}:=\left\{\begin{array}{l|c}
\mathbf{x} \in \mathbb{R}^{\ell(w)+n-1} \mid \begin{array}{cc}
\left(c_{\mathbf{p}}\right)^{t}(\mathbf{x}) \geq 0, & \forall \mathbf{p} \in \mathcal{P}_{\underline{w}}, \\
\left(c_{[i: k]}\right)^{t}(\mathbf{x}) \geq 0, & \forall i \in[n-1], 0 \leq k \leq n_{i}
\end{array}
\end{array}\right\} .
$$

Example 3. Consider $w_{0} \in S_{n}$ and the reduced expression $\underline{\hat{w}}_{0}$ defined above. For $i \in[n-1]$ all GP-paths in $\mathrm{pa}\left(\underline{w}_{0}\right)$ with orientation $\left(l_{i}, l_{i+1}\right)$ are of form

$$
\mathbf{p}_{i, j}:=L_{i} \rightarrow v_{(i, n)} \rightarrow v_{(i, n-1)} \rightarrow \cdots \rightarrow v_{(i, j)} \rightarrow v_{(i+1, j)} \rightarrow \cdots \rightarrow v_{(i+1, n)} \rightarrow L_{i+1} .
$$

In particular, the GP-cone $C_{\underline{w}_{0}}$ is described by inequalities defined by the normal vectors $c_{(i, j+1)}-c_{(i+1, j+1)}$ and $c_{(i, i+1)}$ for $i \in[n-1]$ and $j \in[i+1, n-1]$. The vectors defining weight inequalities are (for all $i<j$ ):

$$
c_{j-i}-c_{(i, j)}-2 \sum_{k=1}^{n-j} c_{(i+k, j+k)}+\sum_{k=0}^{n-j-1} c_{(i+k, j+1+k)}+\sum_{k=0}^{n-j} c_{(i+1+k, j+k)} .
$$

3.4. The (weighted) area cone. We associate to the set of all GP-paths $\mathcal{P}_{\underline{w}}$ a second cone. In this setup, the standard basis of $\mathbb{R}^{\ell(w)+n-1}$ is indexed by the faces of the pseudoline arrangement $\left\{e_{F} \mid F\right.$ face of $\left.\mathrm{pa}(\underline{w})\right\}$. Namely, there are basis vectors associated to faces $F_{(i, j)}$ bounded to the left by a crossing $(i, j)$, and to faces $F_{l}$ unbounded to the left for every $l \in[n-1]$. Let $\mathbf{p} \in \mathcal{P}_{\underline{w}}$. We denote by $\mathrm{A}_{\mathbf{p}}$ the area to the left of $\mathbf{p}$ (with respect to the orientation), i.e. the area enclosed by $\mathbf{p}$. Note that for non-trivial $\mathbf{p}, A_{\mathbf{p}}$ is a non-empty union of faces $F$ in the pseudoline arrangement. We associate to $\mathbf{p}$ the vector

$$
e_{\mathbf{p}}:=-\sum_{F \subset A_{\mathbf{p}}} e_{F} \in \mathbb{R}^{\ell(w)+n-1} .
$$

With a little abuse of notation we denote by $e_{\mathbf{p}}$ also the vector in $\mathbb{R}^{\ell(w)}$ obtained by projecting onto the first $\ell(w)$ coordinates (forgetting the coordinates belonging to the faces that are unbounded to the left, which equal 0 in $e_{\mathbf{p}}$ ).

Definition 11. For a reduced expression $\underline{w} \in S_{n}$, we define the area cone

$$
S_{\underline{w}}:=\left\{\mathbf{x} \in \mathbb{R}^{\ell(w)} \mid\left(e_{\mathbf{p}}\right)^{t}(\mathbf{x}) \geq 0, \forall \mathbf{p} \in \mathcal{P}_{\underline{w}}\right\} .
$$

Again, we are interested in a weighted extension of this cone. For this, we associate to every level $i \in[n-1]$ a union of faces. Consider $F_{i}$, the face of $\mathrm{pa}(\underline{w})$ that is unbounded to the left at level $i$. As before for crossings we set $F_{i_{j}}:=F_{(k, m)}$ if $s_{i_{j}}$ in $\underline{w}$ induces the crossing of $l_{k}$ and $l_{m}$ in $\operatorname{pa}(\underline{w})$. We define $\mathrm{A}_{i}:=F_{i} \cup \bigcup_{k=1}^{n_{i}} F_{i_{k}}$, then $\mathrm{A}_{i} \cap \mathrm{A}_{i^{\prime}}=\varnothing$ if $i \neq i^{\prime}$. It is called the weight area associated to the level $i$. For each $k$ with $0 \leq k \leq n_{i}$, we define a vector

$$
e_{[i: k]}:=-e_{F_{i}}-\sum_{j \in J(i), j \leq j_{k}} e_{F_{i_{j}}} \in \mathbb{R}^{\ell(w)+n} .
$$

Note that $e_{[i: 0]}=-e_{F_{i}}$ and $e_{\left[i: n_{i}\right]}=-\sum_{F \subset A_{i}} e_{F}$.

Definition 12. The weighted area cone $\mathcal{S}_{\underline{w}} \subset \mathbb{R}^{\ell(w)+n-1}$ associated to the reduced expression $\underline{w}$ of $w \in S_{n}$ is defined as

$$
\mathcal{S}_{\underline{w}}:=\left\{\begin{array}{l|l}
\mathbf{x} \in \mathbb{R}^{\ell(w)+n-1} \mid \begin{array}{c}
\left(e_{\mathbf{p}}\right)^{t}(\mathbf{x}) \geq 0, \\
\left(e_{[i: k]}\right)^{t}(\mathbf{x}) \geq 0, \quad \forall i \in[n-1], 0 \leq k \leq n_{i}
\end{array}
\end{array}\right\} .
$$

The additional inequalities induced by the $e_{[i: k]}$ are called weight inequalities. 
Remark 2. In all four cases, $C_{\underline{w}}, \mathcal{C}_{\underline{w}}, S_{\underline{w}}$ and $\mathcal{S}_{\underline{w}}$, some of the inequalities might be redundant and these cones are far from being simplicial in general. The vectors $e_{\mathbf{p}}, c_{\mathbf{p}}, e_{[i: k]}$ and $c_{[i: k]}$ are normal vectors to the defining hyperplanes of the cones $S_{\underline{w}}, C_{\underline{w}}, \mathcal{S}_{\underline{w}}$ and $\mathcal{C}_{\underline{w}}$ respectively. Not all of them are normal vectors to facets of these cones in general.

Example 4. Consider the reduced expression $\underline{\hat{w}}_{0} \in S_{5}$. We have seen all GP-paths in pa $(\underline{w})$ in Example 3. Take the path $\mathbf{p}=L_{1} \rightarrow v_{(1,5)} \rightarrow v_{(1,4)} \rightarrow v_{(1,3)} \rightarrow v_{(1,2)} \rightarrow v_{(2,3)} \rightarrow v_{(2,4)} \rightarrow$ $v_{(2,5)} \rightarrow L_{2}$. The area $A_{\mathbf{p}}$ associated to this path is shaded blue in Figure 6 . The weight area $A_{2}$ corresponding to level 2 is also shown in Figure 6 dotted in red.

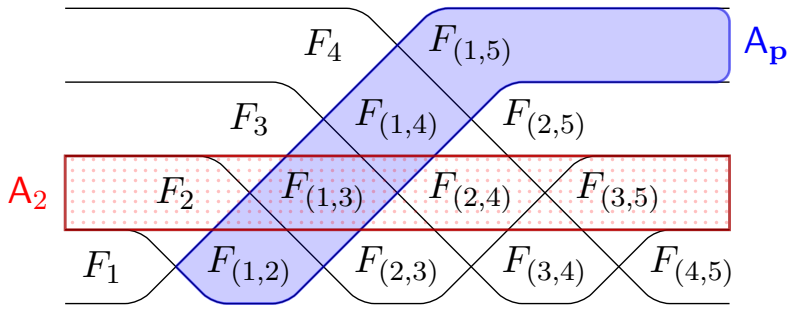

Figure 6 . The area $A_{\mathbf{p}}$ for $\mathbf{p}$ as in Example 4 shaded in blue and the weight area $A_{2}$ dotted in red.

Example 5. Consider $\underline{\hat{w}}_{0} \in S_{n}$ as in Example 3 and recall $\mathbf{p}_{i, j} \in \mathcal{P}_{\underline{w}_{0}}$ with $i \in[n-1]$ and $j \in[i+1, n-1]$. The assigned area is $\mathrm{A}_{\mathbf{p}_{i, j}}=F_{(i, j)} \cup F_{(i, j+1)} \cup \cdots \cup \bar{F}_{(i, n)}$ for $F_{(i, k)}$ the area bounded by $v_{(i, k)}$ to the left. Hence, the cone $S_{\underline{w}_{0}}$ is given by inequalities defined by

$$
e_{\mathbf{p}_{i, j}}=-e_{F_{(i, j)}}-e_{F_{(i, j+1)}}-\cdots-e_{F_{(i, n)}}-e_{F_{(i, n+1)}} .
$$

The additional weight inequalities defining the cone $\mathcal{S}_{\underline{w}_{0}}$ are given by the normal vectors

$$
e_{[i: k]}=-e_{F_{i}}-e_{F_{(1, i+1)}}-e_{F_{(2, i+2)}}-\cdots-e_{F_{(k, i+k)}},
$$

for $i \in[n-1]$ and $0 \leq k \leq n-i$.

3.5. The polytopes. Let $\pi: \mathbb{R}^{\ell(w)+n-1} \rightarrow \mathbb{R}^{n-1}$ be the projection onto the last $n-1$ coordinates, also called weight coordinates. We are interested in the preimage $\pi^{-1}(\lambda)$ for $\lambda \in \mathbb{R}^{n-1}$. It is the intersection of the following hyperplanes for each $i \in[n-1]$ defined by

$$
\left(c_{[i: 0]}\right)^{t}(\mathbf{x})=\lambda_{i}, \forall \mathbf{x} \in \mathbb{R}^{\ell(w)+n-1} .
$$

Fix $w \in S_{n}$ with reduced expression $\underline{w}$. We define a second map $\tau_{\underline{w}}: \mathbb{R}^{\ell(w)+n-1} \rightarrow \mathbb{R}^{n-1}$ by $\tau_{\underline{w}}(\mathbf{x})=\left(\left(e_{\left[i: n_{i}\right]}\right)^{t}(\mathbf{x})\right)_{i=1, \ldots, n-1}$. The preimage of $\lambda \in \mathbb{R}^{n-1}$ with respect to $\tau_{\underline{w}}$ is also an intersection of hyperplanes in $\mathbb{R}^{\ell(w)+n-1}$. For each $i \in[n-1]$ they are defined by

$$
\left(e_{\left[i: n_{i}\right]}\right)^{t}(\mathbf{x})=\lambda_{i}, \forall \mathbf{x} \in \mathbb{R}^{\ell(w)+n-1} .
$$

Definition 13. For $w \in S_{n}$ with reduced expression $\underline{w}$ and for $\lambda \in \mathbb{R}^{n-1}$ we define the following polytopes in $\mathbb{R}^{\ell(w)+n-1}$

$$
\mathcal{S}_{\underline{w}}(\lambda):=\mathcal{S}_{\underline{w}} \cap \tau_{\underline{w}}^{-1}(\lambda) \text { and } \mathcal{C}_{\underline{w}}(\lambda):=\mathcal{C}_{\underline{w}} \cap \pi^{-1}(\lambda) .
$$

Note that by (3.13) (resp. (3.12)) we obtain a description of $\mathcal{S}_{\underline{w}}(\lambda)\left(\right.$ resp. $\left.\mathcal{C}_{\underline{w}}(\lambda)\right)$ in terms of defining equalities and inequalities by replacing the weight inequalities $e_{\left[i: n_{i}\right]}^{t}(\overline{\mathbf{x}}) \geq 0$ in (3.9) (resp. $\left(c_{i}\right)^{t}(\mathbf{x}) \geq 0$ in $\left.(3.5)\right)$ by $\left(e_{\left[i: n_{i}\right]}\right)^{t}(\mathbf{x})=\lambda_{i}\left(\operatorname{resp} . \quad\left(c_{i}\right)^{t}(\mathbf{x})=\lambda_{i}\right)$. In particular, the defining normal vectors for $\mathcal{S}_{\underline{w}}$ (resp. $\mathcal{C}_{\underline{w}}$ ) coincide with those for $\mathcal{S}_{\underline{w}}(\lambda)\left(\operatorname{resp} . \mathcal{C}_{\underline{w}}(\lambda)\right)$. This observation is important in the proof of Theorem 4. 
3.6. A unimodular equivalence. The above pairs of cones (resp. polytopes) $\left(S_{\underline{w}}, C_{\underline{w}}\right)$ and $\left(\mathcal{S}_{\underline{w}}, \mathcal{C}_{\underline{w}}\right)\left(\operatorname{resp} . \quad\left(\mathcal{S}_{\underline{w}}(\lambda), \mathcal{C}_{\underline{w}}(\lambda)\right)\right.$ ) have in fact more in common than the combinatorics defining them. To make this statement precise we need to introduce the notion of unimodular equivalence (see e.g. [HL16, §2]).

Definition 14. Two polytopes $P, Q \subset \mathbb{R}^{d}$ (resp. polyhedral cones $C, D \subset \mathbb{R}^{d}$ ) are called unimodularly equivalent if there exists matrix $M \in G L_{d}(\mathbb{Z})$ and $w \in \mathbb{Z}^{d}$

$$
Q=f_{M}(P)+w\left(\text { resp. } D=f_{M}(C)+w\right),
$$

where $f_{M}(x)=x M$ for $x \in \mathbb{R}^{d}$. We denote this by $Q \cong P($ resp. $C \cong D)$.

This notion of equivalence is of particular interest to us because of its implication on the associated toric varieties. Recall the construction of a projective toric variety $X_{P} \subset \mathbb{P}^{d-1}$ associated with a polytope $P \subset \mathbb{R}^{d}$ in [CLS11, $\S 2.1$ and $\left.\S 2.3\right]$. Then $Q$ being unimodular equivalent to $P$ implies

$$
X_{Q} \cong X_{P}
$$

We want to construct a unimodular equivalence between $\mathcal{C}_{\underline{w}}$ and $\mathcal{S}_{\underline{w}}$ for all reduced expression $\underline{w}$ of $w \in S_{n}$. The following definition is the affine lattice transformation ( $f_{M}$ in Definition 14) that defines the unimodular equivalence. We give it in terms of the bases $\left\{e_{F} \mid F\right.$ face of $\left.\mathrm{pa}(\underline{w})\right\}$ and $\left\{c_{(k, m)}, c_{i} \mid v_{(k, m)} \in \mathrm{pa}(\underline{w})_{0}, i \in[n-1]\right\}$. Morally, we send a face $F$ bounded to the left by a crossing to a linear combination of its adjacent crossings (see (3.17)). A face unbounded to the left is sent to the sum of all crossings at its level.

Definition 15. For $w \in S_{n}$ and $\underline{w}$ a reduced expression we define the linear map $\Psi_{\underline{w}}$ : $\mathbb{R}^{\ell(w)+n-1} \rightarrow \mathbb{R}^{\ell(w)+n-1}$ on the basis $\left\{-e_{F}\right\}$ associated to faces $F$ of pa $(\underline{w})$. Let $F=F_{i_{j_{k}}}$ be the face bounded to the left by the crossing induced from $s_{i_{j_{k}}}=s_{i}$ and $J(i)=\left\{j_{1}, \ldots, j_{n_{i}}\right\}$ (see (3.3)). Then

$$
\Psi_{\underline{w}}\left(-e_{F_{i_{k}}}\right):=c_{i_{j_{k}}}+c_{i_{j_{k+1}}}-\sum_{\substack{j \in J(i-1) \cup J(i+1), j_{k}<j<j_{k+1}}} c_{i_{j}} .
$$

For every level $i \in[n-1]$, we define

$$
\Psi_{\underline{w}}\left(-e_{F_{i}}\right):=c_{[i: 1]} .
$$

Example 6. Consider $\mathrm{pa}(\underline{w})$ for $\underline{w}=s_{1} s_{2} s_{1} \in S_{3}$ as in Figure 4. The two bases for $\mathbb{R}^{5}$ are

$$
\mathcal{B}_{e}=\left\{-e_{F_{1}},-e_{F_{2}},-e_{F_{(1,2)}},-e_{F_{(1,3)}},-e_{F_{(2,3)}}\right\} \text { and } \mathcal{B}_{c}=\left\{c_{1}, c_{2}, c_{(1,2)}, c_{(1,3)}, c_{(2,3)}\right\}
$$

We compute the images of elements in $\mathcal{B}_{e}$ and express them in $\mathcal{B}_{c}$. The coefficients form the columns of the following matrix with the order of the bases as given above.

$$
\left(\begin{array}{ccccc}
1 & 0 & 0 & 0 & 0 \\
0 & 1 & 0 & 0 & 0 \\
-1 & 0 & 1 & 0 & 0 \\
1 & -1 & -1 & 1 & 0 \\
-2 & 1 & 1 & -1 & 1
\end{array}\right) \in G L_{5}(\mathbb{Z})
$$

The observation in the example above is true in general. We obtain the following Lemma as a straightforward consequence of the definition of $\Psi_{\underline{w}}$.

Lemma 1. Let $w \in S_{n}$ with reduced expression $\underline{w}$. Order the bases induced by the faces of $\mathrm{pa}(\underline{w})$ resp. by the crossing points in $\mathrm{pa}(\underline{w})$ as

$$
\mathcal{B}_{e}=\left\{-e_{F_{1}}, \ldots,-e_{F_{n-1}},-e_{F_{i_{1}}}, \cdots-e_{F_{i_{\ell(w)}}}\right\}, \text { resp. } \mathcal{B}_{c}=\left\{c_{1}, \ldots, c_{n-1}, c_{i_{1}}, \ldots, c_{i_{\ell(w)}}\right\} .
$$

Then $\Psi_{\underline{w}}$ can be represented by a lower triangular matrix $M_{\underline{w}}^{e, c}$ with all diagonal entries being 1. In particular, $M_{\underline{w}}^{e, c} \in G L_{\ell(w)+n-1}(\mathbb{Z})$. 
$1 a$

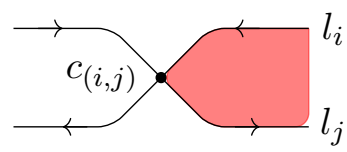

$1 \mathrm{~b}$

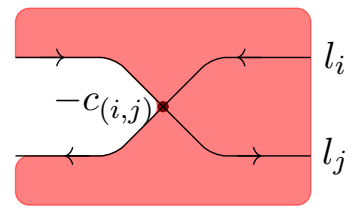

$2 \mathrm{a}$

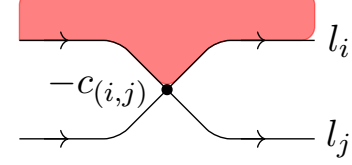

$2 \mathrm{~b}$

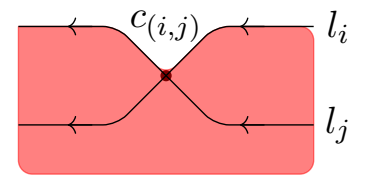

$3 a$

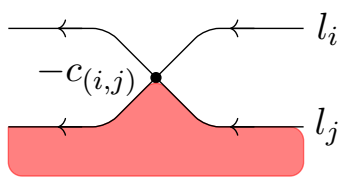

$3 \mathrm{~b}$

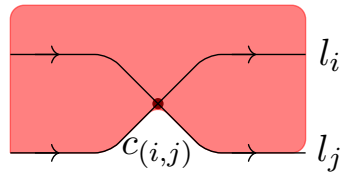

FiguRE 7 . A path $\mathbf{p}$ changing the line at a crossing $(i, j)$ and the corresponding area $A_{p}$.

Corollary 1. With assumptions as in Lemma 1 consider $\left.\Psi_{\underline{w}}\right|_{\mathbb{R}^{\ell(w)}}: \mathbb{R}^{\ell(w)} \rightarrow \mathbb{R}^{\ell(w)}$. We order as before the bases for $\mathbb{R}^{\ell(w)}$ induced by the faces resp. crossing points in $\mathrm{pa}(\underline{w})$ by

$$
\overline{\mathcal{B}}_{e}=\left\{-e_{F_{i_{1}}}, \cdots-e_{F_{i_{\ell(w)}}}\right\}, \text { resp. } \overline{\mathcal{B}}_{c}=\left\{c_{i_{1}}, \ldots, c_{i_{\ell(w)}}\right\} .
$$

Then $\left.\Psi_{\underline{w}}\right|_{\mathbb{R}^{\ell(w)}}$ can be represented by a lower triangular matrix $\bar{M}_{\underline{w}}^{e, c}$ with all diagonal entries 1. In particular, $\bar{M}_{\underline{w}}^{e, c} \in G L_{\ell(w)}(\mathbb{Z})$.

Remark 3. The map $\Psi_{\underline{w}}$ restricted to $\mathbb{R}^{\ell(w)}$ is related to the Chamber Ansatz due to Berenstein-Fomin-Zelevinsky in [BFZ96] (see also [GKS16]).

Proposition 2. Let $w \in S_{n}$ with reduced expression $\underline{w}$. For every $\mathbf{p} \in \mathcal{P}_{\underline{w}}$ we have

$$
\Psi_{\underline{w}}\left(e_{\mathbf{p}}\right)=c_{\mathbf{p}} .
$$

In particular, $\Psi_{\underline{w}}$ sends the normal vector of a defining hyperplane of $\mathcal{S}_{\underline{w}}$ to the normal vector of a defining hyperplane of $\mathcal{C}_{\underline{w}}$.

Proof. We show that for every crossing point $(i, j)$ the coefficient of $c_{(i, j)}$ coincides in $\Psi_{\underline{w}}\left(e_{\mathbf{p}}\right)$ and $c_{\mathbf{p}}$. Recall that $A_{\mathbf{p}}$ is the union of all faces to the left of $\mathbf{p}$ with respect to the given orientation. We distinguish three cases:

(i) If $(i, j)$ lies in the interior of $\mathrm{A}_{\mathbf{p}}$, then four faces $F^{r} \subset \mathrm{A}_{\mathbf{p}}$ with $r \in[4]$ are adjacent to $(i, j)$. For two of them in $\Psi_{\underline{w}}\left(-e_{F^{r}}\right)$ the coefficient of $c_{(i, j)}$ is +1 , for the other two it is -1 . Hence, they cancel each other and in $\Psi_{\underline{w}}\left(-e_{\mathbf{p}}\right)$ it is zero as it is in $c_{\mathbf{p}}$.

(ii) If $\mathbf{p}$ contains $(i, j)$ but does not change the line at $(i, j)$, then $c_{(i, j)}$ has coefficient zero in $c_{\mathbf{p}}$. For $\mathrm{A}_{\mathbf{p}}$, this means that two faces, $F^{1}$ and $F^{2}$, are adjacent to $(i, j)$. One of the two, say $F^{1}$, is bounded by $(i, j)$ to the left where for $F^{2},(i, j)$ is part of the upper or lower boundary. In particular, $\Psi_{\underline{w}}\left(e_{\mathbf{p}}\right)$ contains $c_{(i, j)}$ once with positive and once with negative sign, hence with the coefficient is zero.

(iii) Assume $\mathbf{p}$ changes the line at the crossing $(i, j)$. Figure 7 shows the three possible orientations of $l_{i}$ and $l_{j}$. Each yields two possibilities for the path. If in situation 1a, there is one face $F$ in $\mathrm{A}_{\mathbf{p}}$ bounded by $(i, j)$ to the left. So $c_{(i, j)}$ has coefficient 1 in $\Psi_{\underline{w}}\left(e_{\mathbf{p}}\right)$. As $\mathbf{p}$ changes from $l_{i}$ to $l_{j}$ and $i<j$, also $c_{\mathbf{p}}$ contains $c_{(i, j)}$ with coefficient 1 .

In cases $2 \mathrm{a}$ and $3 \mathrm{a}, \mathrm{A}_{\mathbf{p}}$ contains only one face bounded by $(i, j)$ below resp. above. Hence $c_{(i, j)}$ appears with coefficient -1 in $\Psi_{\underline{w}}\left(e_{\mathbf{p}}\right)$. The same is true for $c_{\mathbf{p}}$ : in both cases $\mathbf{p}$ changes from line $l_{j}$ to $l_{i}$ but $i<j$. 
Three cases remain to be checked, $1 b, 2 b$ and $3 b$ in Figure 7 . In all of them $A_{p}$ contains three faces $F^{1}, F^{2}$ and $F^{3}$ adjacent to $(i, j)$. In case $1 \mathrm{~b},(i, j)$ bounds one face to the left and the other two from above, resp. below. This implies that $c_{(i, j)}$ appears with coefficient -1 in $\Psi_{\underline{w}}\left(e_{\mathbf{p}}\right)$. As $\mathbf{p}$ changes from $l_{j}$ to $l_{i}$ the same is true for $c_{\mathbf{p}}$. For $2 \mathrm{~b}$ and $3 \mathrm{~b}$ we are in the opposite case: two faces in $A_{\mathbf{p}}$ are bounded to the left, resp. right, by $(i, j)$ and only one from above, resp. below. Hence, $\Psi_{\underline{w}}\left(e_{\mathbf{p}}\right)$ contains $c_{(i, j)}$ with coefficient 1 and the same is true for $c_{\mathbf{p}}$, as $\mathbf{p}$ changes from line $l_{i}$ to line $l_{j}$.

For our application later, it remains to show that the normal vectors defining the weight inequalities are mapped onto each other by $\Psi_{\underline{w}}$. Recall the weight area $A_{i}=F_{i} \cup \bigcup_{r=1}^{n_{i}} F_{\left(i_{r}, j_{r}\right)}$ of level $i \in[n-1]$, with $n_{i}$ the number of faces bounded to the left of level $i$.

Proposition 3. Let $w \in S_{n}$ with reduced expression $\underline{w}$. Consider $i \in[n-1]$ with $J(i)=$ $\left\{j_{1}, \ldots, j_{n_{i}}\right\}$. Then for $k \in\left[0, n_{i}\right]$ we have

$$
\Psi_{\underline{w}}\left(e_{[i: k]}\right)=c_{[i: k+1]} \quad \text { and } \quad \Psi_{\underline{w}}\left(e_{\left[i: n_{i}\right]}\right)=c_{[i: 0]} .
$$

In particular, $\Psi_{\underline{w}}$ sends normal vectors of defining (weight) hyperplanes of $\mathcal{S}_{\underline{w}}$ to normal vectors of defining (weight) hyperplanes of $\mathcal{C}_{\underline{w}}$.

Proof. We prove the claim by induction on $k$. By definition we have $\Psi_{\underline{w}}\left(e_{[i: 0]}\right)=\Psi_{\underline{w}}\left(-e_{F_{i}}\right)=$ $c_{[i: 1]}$. Let $1 \leq k<n_{i}-1$, then using induction for the third equation, we obtain

$$
\begin{aligned}
\Psi_{\underline{w}}\left(e_{[i: k+1]}\right) & \stackrel{(3.8)}{=} \Psi_{\underline{w}}\left(e_{[i: k]}-e_{F_{i_{k+1}}}\right) \\
& \stackrel{(3.16)}{=} c_{[i: k+1]}+c_{i_{j_{k+1}}}+c_{i_{j_{k+2}}}-\sum_{\substack{j \in J(i-1) \cup J(i+1), j_{k+1}<j<j_{k+2}}} c_{i_{j}} \\
& \stackrel{(3.4)}{=} c_{i}-c_{i_{j_{k+2}}}-2 \sum_{j \in J(i), j>j_{k+2}} c_{i_{j}}+\sum_{\substack{j \in J(i-1) \cup J(i+1), j>j_{k+2}}} c_{i_{j}}=c_{[i: k+2]} .
\end{aligned}
$$

Now consider $e_{\left[i: n_{i}\right]}=e_{\left[i: n_{i}-1\right]}-e_{F_{i_{j_{i}}}}$. We apply $\Psi_{\underline{w}}$ and obtain the following by induction.

$$
\begin{aligned}
\Psi\left(e_{\left[i: n_{i}\right]}\right) & \stackrel{(3.8)}{=} \Psi_{\underline{w}}\left(e_{\left[i: n_{i}-1\right]}-e_{F_{j_{n_{i}}}}\right) \\
& \stackrel{(3.16)}{=} c_{\left[i: n_{i}\right]}+c_{i_{j_{i}}}-\sum_{\substack{j \in J(i-1) \cup J(i+1), j n_{n_{i}}<j}} c_{i_{j}} \stackrel{(3.4)}{=} c_{i} .
\end{aligned}
$$

We can now prove the first Theorem of this section. It is a more precise formulation of Theorem 1 as stated in the introduction.

Theorem 4. Let $w \in S_{n}$ and $\underline{w}$ a reduced expression. The following polyhedral objects are unimodularly equivalent

(i) $\mathcal{S}_{\underline{w}} \cong \mathcal{C}_{\underline{w}}$ via $\Psi_{\underline{w}}$,

(ii) $\underline{S_{\underline{w}}} \cong \widetilde{C_{\underline{w}}}$ via $\left.\underline{\Psi_{\underline{w}}}\right|_{\mathbb{R}^{\ell(w)}}$,

(iii) $\underline{\mathcal{S}_{\underline{w}}}(\lambda) \cong \mathcal{C}_{\underline{w}}(\lambda)$ for all $\lambda \in \mathbb{R}^{n-1}$ via $\Psi_{\underline{w}}$. 
Proof. We begin by proving (i). From Proposition 2 we know that normal vectors $e_{\mathbf{p}}$ of $\mathcal{S}_{\underline{w}}$ for $\mathbf{p} \in \mathcal{P}_{\underline{w}}$ are mapped to the normal vectors $c_{\mathbf{p}}$ of $\mathcal{C}_{\underline{w}}$, i.e. $\Psi_{\underline{w}}\left(e_{\mathbf{p}}\right)=c_{\mathbf{p}}$. Further, from Proposition 3 we know the same is true for the normal vectors $\bar{e}_{[i: k]}$ of $\mathcal{S}_{\underline{w}}$ for $i \in[n-1]$ : we have $\Psi_{\underline{w}}\left(e_{[i: k]}\right)=c_{[i: k+1]}$ for $k<n_{i}$ and $\Psi_{\underline{w}}\left(e_{\left[i: n_{i}\right]}\right)=c_{[i: 0]}$. As the right hand side of all defining inequalities is zero, we deduce that $\Psi_{\underline{w}}\left(\mathcal{S}_{\underline{w}}\right)=\mathcal{C}_{\underline{w}}$. By Lemma $1, \Psi_{\underline{w}}$ is given by a matrix in $G L_{\ell(w)+n-1}(\mathbb{Z})$ and hence, $\mathcal{S}_{\underline{w}} \cong \mathcal{C}_{\underline{w}}$.

To show (ii), note that by the same argument as for (i) we have $\left.\Psi_{\underline{w}}\right|_{\mathbb{R}^{\ell(w)}}\left(S_{\underline{w}}\right)=C_{\underline{w}}$. By Corollary 1 we deduce $S_{\underline{w}} \cong C_{\underline{w}}$.

For (iii) recall that for $\lambda \in \mathbb{R}^{n-1}$ the polytopes $\mathcal{S}_{\underline{w}}(\lambda)$ resp. $\mathcal{C}_{\underline{w}}(\lambda)$ are defined by the same normal vectors as $\mathcal{S}_{\underline{w}}$ resp. $\mathcal{C}_{\underline{w}}$. As above, it is also true that the right hand sides of the defining (in-)equalities coincides for all normal vectors being mapped onto each other. We therefore deduce $\mathcal{S}_{\underline{w}}(\lambda)=\mathcal{C}_{\underline{w}}(\lambda)$.

\section{String COnes, Polytopes And toric Degenerations}

Recall from $\S 2.1$ our notation for $S L_{n}$. In this section we recall string polytopes and string cones introduced by Littelmann in [Lit98] and Berenstein-Zelevinsky in [BZ01] as well as the weighted string cones defined in [Lit98]. We prove using a result from Gleizer-Postnikov in [GP00] that these are exactly $\mathcal{C}_{\underline{w}}(\lambda)$ resp. $C_{\underline{w}}$ and $\mathcal{C}_{\underline{w}}$.

Littelmann [Lit98] introduced in the context of quantum groups and crystal bases the so called (weighted) string cones and string polytopes $Q_{\underline{w}}(\lambda)$. The motivation is to find monomial bases for the Demazure modules $V_{\underline{w}}(\lambda)$ for $w \in \underline{\epsilon} S_{n}$ and $\lambda \in \Lambda^{+}$. Recall that $\left\{f_{\alpha_{i_{1}}}^{m_{i_{1}}} \cdots f_{\alpha_{i_{\ell}(w)}}^{m_{i_{\ell}(w)}} \cdot v_{\lambda} \in V(\lambda) \mid m_{i_{j}} \geq 0\right\}$ is a spanning set for $V_{\underline{w}}(\lambda)$ depending on a reduced expression $\underline{w}=s_{i_{1}} \cdots s_{i_{\ell(w)}}$. Littelmann identifies a linearly independent subset of this spanning set by introducing the notion of adapted string (see [Lit98, p. 4]) referring to a tuple $\left(a_{1}, \ldots, a_{\ell(w)}\right) \in \mathbb{Z}_{\geq 0}^{\ell(w)}$. His basis for $V_{\underline{w}}(\lambda)$ consists of those elements $f_{i_{1}}^{a_{1}} \cdots f_{i_{\ell(w)}}^{a_{\ell(w)}} \cdot v_{\lambda}$ for which $\left(a_{1}, \ldots, a_{\ell(w)}\right)$ is adapted.

For a fixed reduced expression $\underline{w}$ of $w \in S_{n}$ and $\lambda \in \Lambda^{+}$he gives a recursive definition of the the string polytope $Q_{\underline{w}}(\lambda) \subset \mathbb{R}^{\ell(w)}$ ( [Lit98, p. 5], see also [BZ01]). The lattice points $Q_{\underline{w}}(\lambda) \cap \mathbb{Z}^{\ell(w)}$ are the adapted strings for $\underline{w}$ and $\lambda$. The string cone $Q_{\underline{w}} \subset \mathbb{R}^{\ell(w)}$ is the convex hull of all $Q_{\underline{w}}(\lambda)$ for $\lambda \in \Lambda^{+}$. The weighted string cone $\mathcal{Q}_{\underline{w}} \subset \mathbb{R}^{\ell(w)+n-1}$ is defined as

$$
\mathcal{Q}_{\underline{w}}:=\operatorname{conv}\left(\bigcup_{\lambda \in \Lambda^{+}} Q_{\underline{w}}(\lambda) \times\{\lambda\}\right) \subset \mathbb{R}^{\ell(w)+n-1} .
$$

By definition, one obtains the string polytope from the weighted string cone by intersecting it with the hyperplanes given by $\pi^{-1}(\lambda)$ as in (3.12). The lattice points in the weighted string cone for $w=w_{0}$ parametrize a basis of $\mathbb{C}\left[S L_{n} / U\right] \cong \bigoplus_{\lambda \in \Lambda^{+}} V(\lambda)$.

String polytopes are of great interest to us because of Caldero's work [Cal02] in 2002. He defines for a Schubert variety $X_{w}$ a flat family over $\mathbb{A}^{1}$ with generic fibre $X_{w}$ and special fibre a toric variety. The family is given by a construction using Rees algebras (see [Cal02, §0.2]). Although not defined using valuations initially, it was realized this way in [Kav15] and [FFL17]. His main tools are Lusztig's dual canonical basis and the string parametrization due to [BZ01] and [Lit98]. We summarize his results (restricted to the case of $S L_{n}$ ) below.

Let $\underline{w}=s_{i_{1}} \cdots s_{i_{\ell(w)}}$ be a reduced expression of $w \in S_{n}$. We extend $\underline{w}$ to the right to a reduced expression $\underline{w}_{0}=\underline{w} s_{i_{\ell(w)+1}} \cdots s_{i_{N}}$ of $w_{0}$. This extension is not unique but the results are independent of the extension. Caldero realizes the string cone $Q_{\underline{w}}$ for the Demazure module 
$V_{w}(\lambda)$ as a face of the string cone $Q_{\underline{w}_{0}}$. He deduces the following Lemma as a consequence of [Lit98, §1].

Lemma. (see [Lit98], [Cal02, Lemma 3.3]) Let $w \in S_{n}$ with $\underline{w}=s_{i_{1}} \cdots s_{i_{\ell(w)}}$ a reduced expression and choose a reduced expression $\underline{w}_{0}=\underline{w} s_{i_{\ell(w)+1}} \cdots s_{i_{N}}$. Then the weighted string cone $\mathcal{Q}_{\underline{w}}$ is obtained from the weighted string cone $\mathcal{Q}_{\underline{w}_{0}}$ by setting the variables corresponding to $s_{i_{\ell(w)+1}}, \ldots, s_{i_{N}}$ equal to zero.

Caldero defines a filtration $\left(\mathcal{F}_{\leq m}\right)_{m \geq 1}$ on $\mathbb{C}\left[S L_{n} / U\right]$ with associated graded algebra the semi-group algebra $\mathbb{C}\left[\mathcal{Q}_{\underline{w}_{0}} \cap \mathbb{Z}^{N+n-1}\right]$. Using the Lemma, he defines a quotient filtration $\left(\overline{\mathcal{F}}_{\leq m}\right)_{m \geq 1}$ on $\mathbb{C}\left[S L_{n} / U\right] / I_{w}$, where $I_{w}=\bigoplus_{\lambda \in \Lambda^{+}} V_{w}(\lambda)^{\perp}$ (recall $\left.\S 2.1\right)$, i.e.

$$
\mathbb{C}\left[S L_{n} / U\right] / I_{w}=\bigoplus_{\lambda \in \Lambda^{+}} V(\lambda)^{*} / \bigoplus_{\lambda \in \Lambda^{+}} V_{w}(\lambda)^{\perp} .
$$

The semi-group algebra $\mathbb{C}\left[\mathcal{Q}_{\underline{w}} \cap \mathbb{Z}^{\ell(\underline{w})+n-1}\right]$ is the associated graded algebra of the quotient filtration. In particular, he degenerates $X_{w}$ into a toric variety $Y$, whose normalization is the toric variety $X_{\mathcal{Q}_{w}(\lambda)}$ associated to the string polytope $\mathcal{Q}_{\underline{w}}(\lambda)$ for $\lambda \in \Lambda^{++}$.

4.1. Relation to the GP cones. Gleizer and Postnikov developed in [GP00] a combinatorial model to describe string cones $Q_{\underline{w}_{0}}$ non-recursively for every reduced expression $\underline{w}_{0}$ of $w_{0} \in S_{n}$. They use pseudoline arrangements and GP-paths to obtain the following.

Corollary. [GP00, Corollary 5.8] Let $\underline{w}_{0}$ be reduced expression of $w_{0} \in S_{n}$. Then $C_{\underline{w}_{0}}=Q_{\underline{w}_{0}}$.

On our way to showing that a toric variety isomorphic to $X_{\mathcal{Q}_{w}(\lambda)}$ arises in the context of cluster varieties and mirror symmetry, we first generalize Gleizer-Postnikov's result as follows.

Theorem 5. For every $w \in S_{n}$ with reduced expression $\underline{w}$ and every extension $\underline{w}_{0}=$ $\underline{w} s_{i_{\ell(w)}+1} \cdots s_{i_{N}}$ the following polyhedral objects coincide

(i) $\mathcal{C}_{\underline{w}}=\mathcal{Q}_{\underline{w}}$,

(ii) $C_{\underline{w}}=Q_{\underline{w}}$,

(iii) $\mathcal{C}_{\underline{w}}(\lambda)=\mathcal{Q}_{\underline{w}}(\lambda)$ for $\lambda \in \mathbb{R}^{n-1}$.

In order to prove Theorem 5 we show how to obtain $\mathcal{C}_{\underline{w}}$ from restricting $\mathcal{C}_{\underline{w}_{0}}$ for appropriate $\underline{w}_{0}$. The next subsection is dedicated to introducing restricted paths and concludes with the proof of Theorem 5 .

4.2. Restriction of paths. We show that for $\underline{w}_{0}=\underline{w} s_{i_{\ell(w)+1}} \cdots s_{i_{N}}$ we obtain $\mathcal{C}_{\underline{w}}$ from $\mathcal{C}_{\underline{w}_{0}}$ by setting to zero the coordinates corresponding to crossing points $c_{i_{\ell(w)+1}}, \ldots, c_{i_{N}}$ in $\operatorname{pa}\left(\underline{w}_{0}\right)$.

Definition 16. Let $\underline{w}$ be a reduced expression of $w \in S_{n}$ and fix $\underline{w}_{0}=\underline{w} s_{i_{\ell(w)+1}} \cdots s_{i_{N}}$. Consider $\mathbf{p}_{\underline{w}_{0}} \in \mathcal{P}_{\underline{w}_{0}}$ and draw it in $\mathrm{pa}\left(\underline{w}_{0}\right)$. Then cut $\mathrm{pa}\left(\underline{w}_{0}\right)$ in two pieces along a vertical line, such that all crossing points $v_{i_{p}}$ corresponding to $s_{i_{p}}$ with $1 \leq p \leq \ell(w)$ are on the left of the cut and all $v_{i_{q}}$ corresponding to $s_{i_{q}}, \ell(w)<q \leq N$ are on the right (see Figure 8). We define the restriction $\operatorname{res}_{\underline{w}}\left(\mathbf{p}_{\underline{w}_{0}}\right)$ of $\mathbf{p}_{\underline{w}_{0}}$ to $\mathrm{pa}(\underline{w})$ as the part of $\mathbf{p}_{\underline{w}_{0}}$ to the left of the cut.

We label the intersection points of the lines $l_{i}$ with the cutting line by $\hat{L}_{i}$. An alternative way of describing $\operatorname{res}_{\underline{w}}\left(\mathbf{p}_{\underline{w}_{0}}\right)$ is by removing all vertices $v_{(i, j)}$ from it for which $w\left(\alpha_{i, j-1}\right)>0$. Denote by $\operatorname{res}_{\underline{w}}\left(\mathcal{P}_{\underline{w}_{0}}\right)$ the set of all paths in $\mathrm{pa}(\underline{w})$ that appear in a restriction of a path in $\mathcal{P}_{\underline{w}_{0}}$ (counting each path only once). 


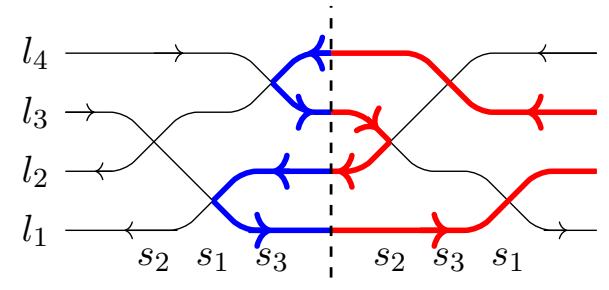

FiguRE 8. A path $\mathbf{p}_{\underline{w}_{0}} \in \mathcal{P}_{\underline{w}_{0}}$ for $\underline{w}_{0}=s_{2} s_{1} s_{3} s_{2} s_{3} s_{1}$ that restricts to two paths $\mathbf{p}_{\underline{w}}, \mathbf{p}_{\underline{w}}^{\prime} \in \mathcal{P}_{\underline{w}}$ (in blue to the left of the dashed cut) for $\underline{w}=s_{2} s_{1} s_{3}$.

Example 7. Consider $\underline{w}=s_{2} s_{1} s_{3}$ and extend it to $\underline{w}_{0}=s_{2} s_{1} s_{3} s_{2} s_{3} s_{1} \in S_{4}$. We draw $p a(\underline{w})$ and endow it with the orientation for $\left(l_{2}, l_{3}\right)$. Figure 8 shows a GP-path $\mathbf{p}_{\underline{w}_{0}}$. Its restriction $\operatorname{res}_{\underline{w}}\left(\mathbf{p}_{\underline{w}_{0}}\right)$ consists of two GP-paths for $\underline{w}$ shown in blue to the left of the cut.

Proposition 4. Let $\underline{w}$ be a reduced expression of $w \in S_{n}$ and fix $\underline{w}_{0}=\underline{w} s_{i_{\ell(w)+1}} \cdots s_{i_{N}}$. Consider $\mathbf{p}_{\underline{w}_{0}} \in \mathcal{P}_{\underline{w}_{0}}$, then $\operatorname{res}_{\underline{w}}\left(\mathbf{p}_{\underline{w}_{0}}\right)$ is either empty or a union of paths in $\mathcal{P}_{\underline{w}}$. In particular, $\operatorname{res}_{\underline{w}}\left(\mathcal{P}_{\underline{w}_{0}}\right) \subset \mathcal{P}_{\underline{w}}$.

Proof. Let $\underline{\mathbf{p}}_{\underline{w}_{0}}$ be a path for orientation $\left(l_{i}, l_{i+1}\right)$, i.e. of form $\underline{\mathbf{p}}_{\underline{w}_{0}}=L_{i} \rightarrow v_{\left(i, j_{1}\right)} \rightarrow v_{\left(j_{1}, j_{2}\right)} \rightarrow$ $\cdots \rightarrow v_{\left(j_{k}, i+1\right)} \rightarrow L_{i+1}$. To simplify notation we set $i=j_{0}$ and $i+1=j_{k+1}$. First note that if $w\left(\alpha_{j_{r}, j_{r+1}-1}\right)>0$ for all $0 \leq r \leq k$ then $\operatorname{res}_{\underline{w}}\left(\mathbf{p}_{\underline{w}_{0}}\right)=\varnothing$. Otherwise $\operatorname{res}_{\underline{w}}\left(\mathbf{p}_{\underline{w}_{0}}\right)$ is a union of paths

$$
\mathbf{p}_{r}=\hat{L}_{j_{r}} \rightarrow v_{\left(j_{r}, j_{r+1}\right)} \rightarrow \cdots \rightarrow v_{\left(j_{r+s}, j_{r+s+1}\right)} \rightarrow \hat{L}_{j_{r+s+1}}
$$

such that $w\left(\alpha_{j_{r+p}, j_{r+p+1}-1}\right)<0$ for all $0 \leq p \leq s, 0 \leq r \leq k$ and $0 \leq s \leq k-r$. By definition, each $\mathbf{p}_{r}$ is rigorous and hence, in $\mathcal{P}_{\underline{w}}$.

We want to show the other implication, $\mathcal{P}_{\underline{w}} \subset \operatorname{res}_{\underline{w}}\left(\mathcal{P}_{\underline{w}_{0}}\right)$. In Algorithm 2 we give a construction to obtain a path in $\mathrm{pa}\left(\underline{w}_{0}\right)$ from a given path in $\overline{\mathcal{P}}_{\underline{w}}$. The following proposition shows that the algorithm always terminates and that the output is in fact a path in $\mathcal{P}_{\underline{w}_{0}}$.

Proposition 5. Algorithm 2 terminates for all $\mathbf{p}_{\underline{w}} \in \mathcal{P}_{\underline{w}}$ and $\operatorname{ind}_{\underline{w}_{0}}\left(\mathbf{p}_{\underline{w}}\right) \in \mathcal{P}_{\underline{w}_{0}}$.

Proof. By Proposition $1 \mathbf{p}_{\underline{w}}$ lies in the region of $\mathrm{pa}(\underline{w})$ in between the lines $l_{i}$ and $l_{i+1}$. In particular, at some point there is a $p^{\prime}$ with $l_{i+m-p-p^{\prime}}=l_{i+1}$ terminating the first loop and a $q^{\prime}$ with $l_{i-l+q+q^{\prime}}=l_{i}$ terminating the second loop.

To see that $\operatorname{ind}_{\underline{w}_{0}}\left(\mathbf{p}_{\underline{w}}\right) \in \mathcal{P}_{\underline{w}_{0}}$ observe that changing the lines as indicated by the algorithm avoids exactly the two situations from Figure 5 forbidden in rigorous paths. Hence, $\operatorname{ind}_{\underline{w}_{0}}\left(\mathbf{p}_{\underline{w}}\right)$ is rigorous.

By Proposition 5 we can define the following.

Definition 17. Let $\underline{w}$ be a reduced expression of $w \in S_{n}$ and fix $\underline{w}_{0}=\underline{w} s_{i_{\ell(w)+1}} \cdots s_{i_{N}}$. For $\mathbf{p}_{\underline{w}} \in \mathcal{P}_{\underline{w}}$ we define the induced path $\operatorname{ind}_{\underline{w}_{0}}\left(\mathbf{p}_{\underline{w}}\right) \in \mathcal{P}_{\underline{w}_{0}}$ as the output of Algorithm 2 .

Example 8. Consider $\underline{w}=s_{2} s_{1} s_{3}$ and extend it to $\underline{w}_{0}=s_{2} s_{1} s_{3} s_{2} s_{3} s_{1} \in S_{4}$. We draw $\operatorname{pa}(\underline{w})$ and endow it with the orientation for $\left(l_{2}, l_{3}\right)$. Figure 9 shows a GP-path $\mathbf{p}_{\underline{w}}$ in blue to the left of the cut. The extension of $\mathbf{p}_{\underline{w}}$ in red to the right of the cut completes $\mathbf{p}_{\underline{w}}$ to the induced path $\operatorname{ind}_{\underline{w}_{0}}\left(\mathbf{p}_{\underline{w}}\right)$ that is the output of Algorithm 2 .

Proposition 6. Let $\underline{w}$ be a reduced expression of $w \in S_{n}$ and fix $\underline{w}_{0}=\underline{w} s_{i_{\ell(w)}+1} \cdots s_{i_{N}}$. For every $\mathbf{p}_{\underline{w}} \in \mathcal{P}_{\underline{w}}$ there exists $\mathbf{p}_{\underline{w}_{0}} \in \mathcal{P}_{\underline{w}_{0}}$ such that, $\operatorname{res}_{\underline{w}}\left(\mathbf{p}_{\underline{w}_{0}}\right)=\mathbf{p}_{\underline{w}}$. In particular, we have $\mathcal{P}_{\underline{w}} \subset \operatorname{res}_{\underline{w}}\left(\mathcal{P}_{\underline{w}_{0}}\right)$. 


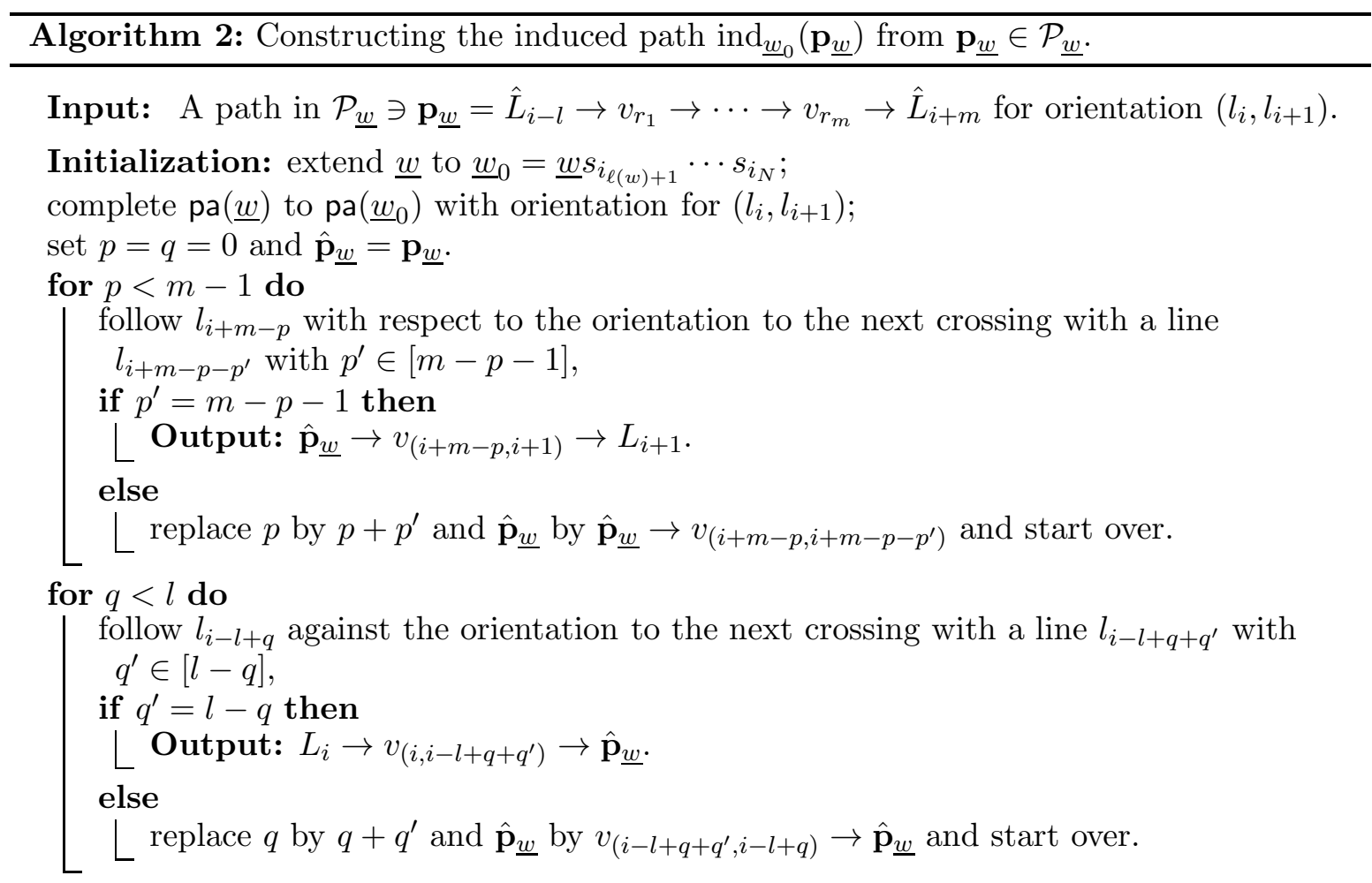

Output: A path $\operatorname{ind}_{\underline{w}_{0}}\left(\mathbf{p}_{\underline{w}}\right):=L_{i} \rightarrow v_{(i, i-l+q)} \rightarrow \cdots \rightarrow \mathbf{p}_{\underline{w}} \rightarrow \cdots \rightarrow v_{(i+m-p, i+1)} \rightarrow L_{i+1}$.

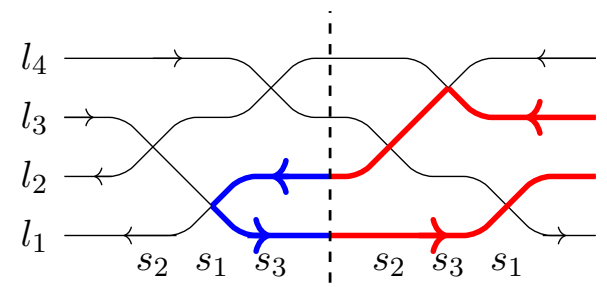

Figure 9. A path $\mathbf{p}_{\underline{w}} \in \mathcal{P}_{\underline{w}}$ for $\underline{w}=s_{2} s_{1} s_{3}$ and the induced path $\operatorname{ind}_{\underline{w}_{0}}\left(\mathbf{p}_{\underline{w}}\right) \in$ $\mathcal{P}_{\underline{w}_{0}}$ with $\underline{w}_{0}=\underline{w} s_{2} s_{3} s_{1}$.

Proof. By construction $\operatorname{ind}_{\underline{w}_{0}}\left(\mathbf{p}_{\underline{w}}\right) \in \mathcal{P}_{\underline{w}_{0}}$ satisfies $\operatorname{res}_{\underline{w}}\left(\operatorname{ind}_{\underline{w}_{0}}\left(\mathbf{p}_{\underline{w}}\right)\right)=\mathbf{p}_{\underline{w}}$.

Recall for $i \in[n-1]$ the definition $J(i)$ and $n_{i}$ from (3.3). To distinguish between the sets for $\underline{w}$ and $\underline{w}_{0}$, we use the notation $J(i) \underline{\underline{w}}$ (resp. $\left.J(i) \underline{\underline{w}}_{0}\right)$ and $n_{i}^{\underline{w}}\left(\operatorname{resp} \cdot n_{i}^{\underline{w}_{0}}\right)$. We define the following polyhedral objects from restricted paths and show they equal the (weighted) GP-cone, respectively polytope, in the subsequent key proposition for proving Theorem 5 .

Definition 18. Let $\underline{w}$ be a reduced expression of $w \in S_{n}$ and fix $\underline{w}_{0}=\underline{w} s_{i_{\ell(w)}+1} \cdots s_{i_{N}}$. We define the restricted weighted GP-cone as

$$
\operatorname{res}_{\underline{w}}\left(\mathcal{C}_{\underline{w}_{0}}\right):=\left\{\mathbf{x} \in \mathbb{R}^{\ell(w)+n-1} \mid \begin{array}{cc}
\left(c_{\operatorname{res}_{\underline{w}}\left(\mathbf{p}_{\underline{w}_{0}}\right)}\right)^{t}(\mathbf{x}) \geq 0, & \forall \mathbf{p}_{\underline{w}_{0}} \in \mathcal{P}_{\underline{w}_{0}}, \\
\left(c_{[i: k]}\right)^{t}(\mathbf{x}) \geq 0, & \forall i \in[n-1], 0 \leq k \leq n \underline{w}
\end{array}\right\} .
$$


Similarly, we define $\operatorname{res}_{\underline{w}}\left(C_{\underline{w}_{0}}\right):=\left\{\mathbf{x} \in \mathbb{R}^{\ell(w)} \mid\left(c_{\operatorname{res}_{\underline{w}}\left(\mathbf{p}_{\underline{w}_{0}}\right)}\right)^{t}(\mathbf{x}) \geq 0, \forall \mathbf{p}_{\underline{w}_{0}} \in \mathcal{P}_{\underline{w}_{0}}\right\}$ the restricted $G P$-cone and the polytope $\operatorname{res}_{\underline{w}}\left(\mathcal{C}_{\underline{w}_{0}}(\lambda)\right):=\operatorname{res}_{\underline{w}}\left(\underline{\mathcal{C}}_{\underline{w}_{0}}\right) \cap \pi^{-1}(\lambda)$ (see (3.12)) for $\lambda \in \mathbb{R}^{n-1}$.

Proposition 7. For every $w \in S_{n}$ with reduced expression $\underline{w}$ and every extension $\underline{w}_{0}=$ $\underline{w} s_{i_{\ell(w)}+1} \cdots s_{i_{N}}$ the following polyhedral objects coincide

(i) $\mathcal{C}_{\underline{w}}=\operatorname{res}_{\underline{w}}\left(\mathcal{C}_{\underline{w}_{0}}\right)$,

(ii) $\overline{C_{\underline{w}}}=\operatorname{res}_{\underline{w}}\left(\bar{C}_{\underline{w}_{0}}\right)$,

(iii) $\underline{\mathcal{C}_{\underline{w}}}(\lambda)=\overline{\operatorname{res}}_{\underline{w}}\left(\underline{\mathcal{C}}_{\underline{w}_{0}}(\lambda)\right)$ for $\lambda \in \mathbb{R}^{n-1}$.

Proof. We start by showing (i), then (ii) and (iii) are direct implications. Note that only the inequalities induced by GP-paths differ in the definition of $\mathcal{C}_{\underline{w}}(3.5)$, resp. $\operatorname{res}_{\underline{w}}\left(\mathcal{C}_{\underline{w}_{0}}\right)(4.1)$. By Proposition 4 we have $\mathcal{C}_{\underline{w}} \subseteq \operatorname{res}_{\underline{w}}\left(\mathcal{C}_{\underline{w}_{0}}\right)$. By Proposition 6 we deduce $\operatorname{res}_{\underline{w}}\left(\mathcal{C}_{\underline{w_{0}}}\right) \subseteq \mathcal{C}_{\underline{w}}$ and hence, equality follows.

We have now collected all ingredients necessary to provide the proof of Theorem 5 .

Proof of Theorem 5. We show $\mathcal{Q}_{\underline{w}}=\operatorname{res}_{\underline{w}}\left(\mathcal{C}_{\underline{w}_{0}}\right)$ for every extension $\underline{w}_{0}=\underline{w} s_{i_{\ell(w)+1}} \cdots s_{i_{N}}$ and then apply Proposition 7. By [Cal02, Lemma 3.3] (restated above) we know that

$$
\mathcal{Q}_{\underline{w}}=\mathcal{Q}_{\underline{w}_{0}} \cap \bigcap_{(i, k): w\left(\alpha_{i, k-1}\right)>0}\left\{x_{(i, k)}=0\right\},
$$

as the $x_{(i, k)}$ appearing in the intersection of hyperplanes on the right correspond to the coordinates $x_{s_{p}}$ with $\ell(w)<p \leq N$ in the extension of $\underline{w}$ to $\underline{w}_{0}$. Further, we observe that if $c_{\mathbf{p}_{\underline{w} 0}}=\sum_{k} c_{\left(i_{k}, j_{k}\right)}$ then $c_{\operatorname{res}_{\underline{w}}\left(\mathbf{p}_{\underline{w}_{0}}\right)}=\sum_{k: w\left(\alpha_{\left.i_{k}, j_{k}-1\right)>0}\right.} c_{\left(i_{k}, j_{k}\right)}$. Regarding the normal vectors for weight inequalities $c_{[i: k]}$ (see (3.4)), observe that for $k>n \underline{w}$ we obtain $c_{i}$ from $c_{[i: k]}$ when setting those $c_{\left(i_{k}, j_{k}\right)}$ to zero with $w\left(\alpha_{i_{k}, j_{k}-1}\right)>0$. Hence, $\mathcal{Q}_{\underline{w}}=\operatorname{res}_{\underline{w}}\left(\mathcal{C}_{\underline{w}_{0}}\right)=\mathcal{C}_{\underline{w}}$ by Propositon 7 . Then $Q_{\underline{w}}=C_{\underline{w}}$ is a direct consequence and identifying $\Lambda^{+}$with $\mathbb{R}^{n-1}$ using the fundamental weights, (iii) follows.

\section{Double Bruhat Cells and the superpotential}

Recall the notation for cluster varieties from above. In this section we explain the $\mathcal{A}$-cluster variety that can be associated to the quiver from a pseudoline arrangement. Based on results of Berenstein-Fomin-Zelevinsky this variety is a double Bruhat cell (see Definition 19). We apply the construction of [GHKK18] (see §2.2) and recall results of Magee in [Mag15] regarding the superpotential.

Recall that $S L_{n}$ has two cell decompositions (the Bruhat decompositions) in terms of Bruhat cells indexed by elements of the symmetric group

$$
S L_{n}=\bigcup_{u \in S_{n}} B u B=\bigcup_{v \in S_{n}} B^{-} v B^{-} .
$$

Definition 19. The double Bruhat cell associated to $e$ and $w$ in $S_{n}$ is

$$
G^{e, w}:=B \cap B^{-} w B^{-} \subset S L_{n} .
$$

The cluster structure of $G^{e, w}$ can be established as follows. Choose a reduced expression $\underline{w}$ and consider $\mathrm{pa}(\underline{w})$. Recall from Definition 6 that every face of $\mathrm{pa}(\underline{w})$ corresponds to a vertex of $Q_{\underline{w}}$. We therefore associate cluster variables to faces of $\operatorname{pa}(\underline{w})$. Let $F$ be such a face and assume the lines $l_{j_{1}}, \ldots, l_{j_{k}}$ pass below $F$. In particular, $F$ is of level $k$. Then associate the Plücker coordinate $\bar{p}_{j_{1}, \ldots, j_{k}} \in \mathbb{C}\left[S L_{n}\right]$ to $F$, i.e. the minor of the columns $[k]$ and rows $\left\{j_{1}, \ldots, j_{k}\right\}$. To remember it was associated with $F$, we set $A_{F}:=\bar{p}_{j_{1}, \ldots, j_{k}}$. 
Definition 20. Let $w \in S_{n}$ with reduced expression $\underline{w}$. Then the quiver $Q_{\underline{w}}$ together with the set of cluster variables $\mathbf{A}_{\underline{w}}:=\left\{A_{F} \mid F\right.$ a face of $\left.\mathrm{pa}(\underline{w})\right\}$ forms the seed $s_{\underline{w}}:=\left(\mathbf{A}_{\underline{w}}, Q_{\underline{w}}\right)$.

Example 9. Recall from Example 1 and Figure 3 the pseudoline arrangement pa $\left(\underline{\hat{w}}_{0}\right)$ and the quiver $Q_{\hat{\underline{w}}_{0}}$ for $\underline{\hat{w}}_{0} \in S_{5}$. To a face $F_{(i, j)}$ with $i \in[n-1]$ and $j \in[i+1, n]$ we associate following the above recipe the cluster variable $A_{(i, j)}:=\bar{p}_{i+1, \ldots, j}$. To the faces unbounded $F_{i}, i \in[4]$ to the left, we associate the variables $A_{i}:=\bar{p}_{5-i+1, \ldots, 5}$. Note that the variables associated to the frozen vertices on the left (from bottom to top) are $\bar{p}_{5}, \bar{p}_{45}, \bar{p}_{345}, \bar{p}_{2345}$ and those associated to the frozen vertices on the right are $\bar{p}_{1}, \bar{p}_{12}, \bar{p}_{123}, \bar{p}_{1234}$. These Plücker coordinates are called consecutive minors. The collection of all cluster variables associated to this initial seed is

$$
\mathbf{A}_{\underline{\hat{w}}_{0}}=\left\{\bar{p}_{1}, \bar{p}_{2}, \bar{p}_{3}, \bar{p}_{4}, \bar{p}_{5}, \bar{p}_{12}, \bar{p}_{23}, \bar{p}_{34}, \bar{p}_{45}, \bar{p}_{123}, \bar{p}_{234}, \bar{p}_{345}, \bar{p}_{1234}, \bar{p}_{2345}\right\} \text {. }
$$

Example 10. Consider $\underline{\hat{w}}_{0} \in S_{n}$ as in Examples 3 and 5. Then the collection $\mathbf{A}_{\underline{\hat{w}}_{0}}$ of associated cluster variables is

$$
\mathbf{A}_{\underline{\hat{w}}_{0}}=\left\{\bar{p}_{i, \ldots, j} \mid i \in[n-1], j \in[i, n]\right\},
$$

where $\bar{p}_{i, \ldots, j}$ is a frozen variable if either $i=1$ or $j=n$. Note that $\bar{p}_{1, \ldots, n}=$ det, which is constant on $S L_{n}$, hence we disregard it. From now on we denote by $s_{0}$ the seed $s_{0}:=s_{\underline{\hat{w}}_{0}}=$ $\left(\mathbf{A}_{\underline{\hat{w}}_{0}}, Q_{\underline{\hat{w}}_{0}}\right)$.

Berenstein-Fomin-Zelevinsky showed

Theorem. ( [BFZ05, Theorem 2.10]) Let $w \in S_{n}$ with reduced expression $\underline{w}$. Then for the upper cluster algebra $\overline{\mathcal{Y}}\left(s_{\underline{w}}\right)$ one has an isomorphism of algebras

$$
\overline{\mathcal{Y}}\left(s_{\underline{w}}\right) \otimes_{\mathbb{Z}} \mathbb{C} \cong \mathbb{C}\left[G^{e, w}\right] .
$$

In particular, the Theorem implies the following: if $\underline{w}_{1}$ and $\underline{w}_{2}$ are two reduced expressions of $w \in S_{n}$ related by mutation in the sense of Definition 7 , then the associated seeds $s_{\underline{w}_{1}}$ and $s_{\underline{w}_{2}}$ are related by cluster $(\mathcal{A}$-)mutation in the sense of (2.4). This explains our abuse of notation using the same letter $\mu$ for both types of mutation.

We now focus on the $\mathcal{A}$-cluster variety $G^{e, w_{0}}$ and the natural partial compactification using the frozen variables to study the superpotential as in $\S 2.2$. We partially compactify $G^{e, w_{0}}$ to $\bar{G}^{e, w_{0}}$ by allowing the frozen variables $\bar{p}_{[i]}$ and $\bar{p}_{[n-i, n]}$ for $i \in[n-1]$ to vanish. Denote the resulting boundary divisor $D \subset \bar{G}^{e, w_{0}}$ and its irreducible components by

$$
D_{i}:=\left\{\bar{p}_{[i]}=0\right\}, \text { resp. } D_{i, n}:=\left\{\bar{p}_{[n-i, n]}=0\right\} .
$$

There is an open embedding $G^{e, w_{0}} \hookrightarrow S L_{n} / U$ given by $g \mapsto g^{t} U$ and up to codimension 2 the variety $\bar{G}^{e, w_{0}}$ agrees with $S L_{n} / U$ (this follows, for example, from [Mag15, Proposition 23]). Hence, we have an isomorphism of rings $\mathbb{C}\left[\bar{G}^{e, w_{0}}\right] \cong \mathbb{C}\left[S L_{n} / U\right]$. One of Magee's main results in $[$ Mag15] is the following.

Theorem. ( [Mag15, Corollary 3]) The full Fock-Goncharov conjecture holds for $S L_{n} / U$.

Moreover, Magee shows that there exists an optimized seed for every frozen vertex and therefore we can apply Algorithm 1 stated in $\S 2.2$ to compute the superpotential. This is indeed what Magee did for the initial seed $s_{0}$ (see Example 10). Let $\mathcal{X}$ denote the FockGoncharov dual to the $\mathcal{A}$-cluster variety $G^{e, w_{0}}$ (see Definition 3 ). Recall that in the initial seed $s_{0}$ we have $N_{s_{0}} \cong \mathbb{Z}^{N+n-1}$ with basis $\left\{e_{F} \mid F\right.$ face of pa $\left.\left(\underline{w}_{0}\right)\right\}$. As before we set $e_{F_{(i, j)}}=: e_{(i, j)}$ and $e_{F_{k}}=: e_{k}$. Further, recall that the superpotential $W: \mathcal{X} \rightarrow \mathbb{C}$ is given by the sum of $\vartheta$-functions associated to frozen variables. We denote by $\vartheta_{i}$ (resp. $\vartheta_{(i, n)}$ ) the $\vartheta$-functions associated to the frozen vertex $w_{i}\left(\right.$ resp. $\left.w_{(i, n)}\right)$ in the initial quiver $Q_{s_{0}}$ (see Figure 3 ) for $i \in[n-1]$. 
Proposition. ( $[$ Mag15, Corollary 24]) Let $W: \mathcal{X} \rightarrow \mathbb{C}$ denote the superpotential. Then we have $\left.W\right|_{\mathcal{X}_{s_{0}}}=\left.\sum_{i=1}^{n-1} \vartheta_{i}\right|_{\mathcal{X}_{s_{0}}}+\vartheta_{(n-i, n)} \mid \mathcal{X}_{s_{0}}$, where

$$
\left.\vartheta_{i}\right|_{\mathcal{X}_{s_{0}}}=\sum_{k=0}^{n-1-i} z^{-e_{i}-\sum_{j=1}^{k} e_{(j, i+j)}}, \text { and }\left.\vartheta_{(i, n)}\right|_{\mathcal{X}_{s_{0}}}=\sum_{k=0}^{n-1-i} z^{-\sum_{j=0}^{k} e_{(i, n-j)}} \text {, for } i \in[n-1] \text {. }
$$

Example 11. Consider $S_{3}$ and the initial seed with quiver $Q_{s_{1} s_{2} s_{1}}$. Then

$$
\begin{aligned}
\left.W\right|_{\mathcal{X}_{s_{0}}} & =\vartheta_{(1,3)}+\vartheta_{(2,3)}+\vartheta_{1}+\vartheta_{2} \\
& =z^{-e_{(1,3)}}+z^{-e_{(1,3)}-e_{(1,2)}}+z^{-e_{(2,3)}}+z^{-e_{1}}+z^{-e_{1}-e_{(1,2)}}+z^{-e_{2}} .
\end{aligned}
$$

Definition 21. For $\underline{w}_{0}$ a reduced expression of $w_{0} \in S_{n}$ we define the following polyhedral objects by tropicalizing a sum of $\vartheta$-functions resp. the superpotential:

$$
\begin{aligned}
\Xi_{\underline{w}_{0}} & :=\left\{\mathbf{x} \in \mathbb{R}^{N+n-1}|W|_{\mathcal{X}_{\underline{w}_{0}}}^{\text {trop }}(\mathbf{x}) \geq 0\right\}, \\
\Xi_{\underline{w}_{0}} & :=\left\{\mathbf{x} \in \mathbb{R}^{N} \mid\left(\sum_{i=1}^{n-1} \vartheta_{(i, n)} \mid \mathcal{X}_{\underline{w}_{0}}\right)^{\operatorname{trop}}(\mathbf{x}) \geq 0\right\}, \\
\Xi_{\underline{w}_{0}}(\lambda) & :=\Xi_{\underline{w}_{0}} \cap \tau_{\underline{w}_{0}}^{-1}(\lambda) \text { for } \lambda \in \mathbb{R}^{n-1} .
\end{aligned}
$$

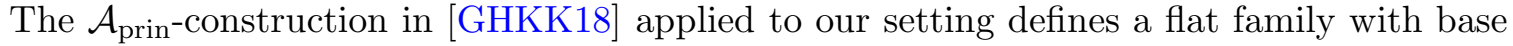
$\mathbb{A}^{N-2 n+2}$ for every choice of seed, in particular for every $\underline{w}_{0}$. The central fibre is by [GHKK18, Theorem 8.39] the toric variety associated to $\Xi_{\underline{w}_{0}}(\lambda)$ for $\lambda \in \mathbb{Z}_{>0}^{n-1}$. One generic fibre is $S L_{n} / B$, hence we have a toric degeneration of the flag variety. We do not go into the details on this construction but refer the reader to [GHKK18, §8].

5.1. Relating to the area cones. Let $\underline{w}_{0}$ be an arbitrary reduced expression of $w_{0} \in S_{n}$. In what follows we show how to obtain an expression of the superpotential in any seed $s_{\underline{w}_{0}}$ associated to $\underline{w}_{0}$ by "detropicalizing" the weighted cone $\mathcal{S}_{\underline{w}_{0}}$. We define it more generally for $\underline{w}$ a reduced expression of $w \in S_{n}$. Denote by $\mathcal{X}_{\underline{w}}$ the cluster torus associated to the seed $s_{\underline{w}}$.

Definition 22. Let $\underline{w}$ be an arbitrary reduced expression of $w \in S_{n}$. Then the detropicalization of the GP data, respectively the cone $\mathcal{S}_{\underline{w}}$ is defined as the function $W_{\mathcal{S}_{\underline{w}}}: \mathcal{X}_{\underline{w}} \rightarrow \mathbb{C}$ with

$$
W_{\mathcal{S}_{\underline{w}}}:=\sum_{\mathbf{p} \in \mathcal{P}_{\underline{w}}} z^{e^{\mathbf{p}}}+\sum_{i \in[n-1], 0 \leq k \leq n_{i}} z^{e^{[i: k]}}
$$

The name is self-explanatory, observe that by definition we have

$$
\left\{\mathbf{x} \in \mathbb{R}^{\ell(w)+n-1} \mid W_{\mathcal{S}_{\underline{w}}}^{\text {trop }}(\mathbf{x}) \geq 0\right\}=\mathcal{S}_{\underline{w}} .
$$

Proposition 8. Let $\underline{\hat{w}}_{0}=s_{1} s_{2} s_{1} \cdots s_{n-1} s_{n-2} \cdots s_{2} s_{1}$ be the reduced expression associated to the initial seed $s_{0}$ as above. Then $W_{\mathcal{S}_{\underline{\underline{\hat{w}}}}}=\left.W\right|_{\mathcal{X}_{s_{0}}}$.

Proof. Recall from Example 5 the expressions $e_{\mathbf{p}_{i, j}}$ (3.10) and $e_{[i: k]}(3.11)$ for $i \in[n-1]$ and $j, k \in[i+1, n]$. In comparison with [Mag15, Corollary 24] (restated above) we obtain

$$
\vartheta_{(i, n)} \mid \mathcal{X}_{s_{0}}=\sum_{j=i+1}^{n} z^{e_{\mathbf{p}_{i, j}}}, \text { and }\left.\vartheta_{i}\right|_{\mathcal{X}_{s_{0}}}=\sum_{k=0}^{n-1-i} z^{e_{[i: k]}}
$$

As from Example 3 we know $\mathcal{P}_{\underline{w}_{0}}=\left\{\mathbf{p}_{i, j} \mid i \in[n-1], j \in[i+1, n]\right\}$, the claim follows. 


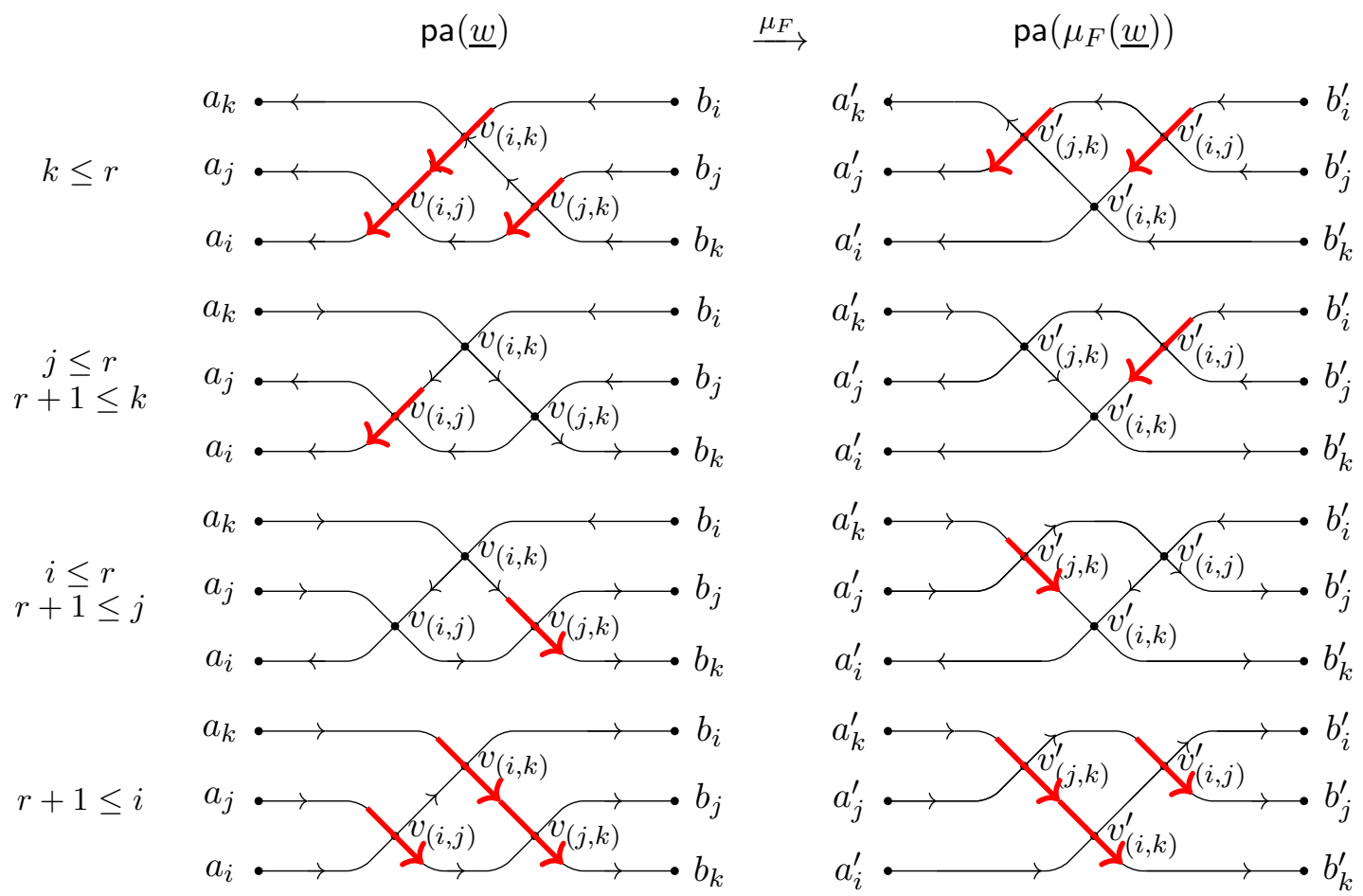

Figure 10. The pseudoline arrangement $\operatorname{pa}(\underline{w})$ (resp. $\operatorname{pa}\left(\mu_{F}(\underline{w})\right)$ ) locally around the face $F=F_{(i, j)}$ (resp. $\left.F^{\prime}=F_{(j, k)}^{\prime}\right)$ bounded by lines $l_{i}, l_{j}, l_{k}$ with $i<j<k$ and orientations $\left(l_{r}, l_{r+1}\right)$ for all possible $r$. The red arrows are those forbidden in GP-paths.

5.2. Mutation of the area cone. Our aim is to generalize Proposition 8 for arbitrary reduced expressions $\underline{w}_{0}$. We achieve this by showing that the detropicalization of $\mathcal{S}_{\underline{w}_{0}}$ behaves as the superpotential does when applying $\mathcal{X}$-mutation. Further, we show that if $\mu(\underline{w})$ and $\underline{w}$ are reduced expressions of $w \in S_{n}$, then $\mu^{*}\left(W_{\mathcal{S}_{\mu(\underline{w})}}\right)=W_{\mathcal{S}_{\underline{w}}}$, where $\mu^{*}: \mathbb{C}\left[\mathcal{X}_{\mu(\underline{w})}\right] \rightarrow \mathbb{C}\left[\mathcal{X}_{\underline{w}}\right]$ is the pull-back of the cluster mutation as in (2.3). This follows from Lemma 3 and Lemma 4. Recall from Definition 7 the mutation of pseudoline arrangements. The core of this subsection is the case-by-case analysis of how mutation effects GP-paths.

In Figure 10 we display locally around the mutable face $F=F_{(i, j)}$ (resp. $F^{\prime}$ ) the orientations of $\mathrm{pa}(\underline{w})$ (resp. $\mathrm{pa}\left(\mu_{F}(\underline{w})\right)$ ). The red arrows indicate which passages are forbidden in GPpaths. In Tables 1 to 4 we list in the second column all possibilities how a GP-path $\mathbf{p}$ locally looks around the face $F$. In the third column of each table is a complete list of how GP-paths look locally around the face $F^{\prime}$ obtained from $F$ by mutation $\mu_{F}$.

Recall the arrows for the quiver corresponding to $\mathrm{pa}(\underline{w})$ and $\mathrm{pa}\left(\mu_{F}(\underline{w})\right)$ from Figure 2 . We call a face $E$ incoming (resp. outgoing) with respect to $F$ in $\mathrm{pa}(\underline{w})$, if there is an arrow in the quiver $Q_{\underline{w}}$ from (resp. to) the vertex corresponding to $E$ to (resp. from) the vertex corresponding to $F$. We denote by $\operatorname{In}_{F}$ the union of all incoming faces and by $\mathrm{Out}_{F}$ the union of all outgoing faces. See for example, Figure 2. 


\begin{tabular}{|c|c|c|c|}
\hline$F$-local type of $\mathbf{p}$ & $\mathbf{p}$ in pa $(\underline{w})$ & $\mathbf{p}^{\prime}=\operatorname{mut}_{F}(\mathbf{p})$ in pa $\left(\mu_{F}(\underline{w})\right)$ & $F^{\prime}$-local type of $\mathbf{p}^{\prime}$ \\
\hline \hline$(2,1,2)$ & $b_{i} \rightarrow v_{(i, k)} \rightarrow a_{k}$ & $b_{i}^{\prime} \rightarrow v_{(i, j)}^{\prime} \rightarrow v_{(j, k)}^{\prime} \rightarrow a_{k}^{\prime}$ & $(2,1,2)$ \\
\hline$(1,1,2)$ & $b_{j} \rightarrow v_{(j, k)} \rightarrow v_{(i, k)} \rightarrow a_{k}$ & $\frac{b_{j}^{\prime} \rightarrow v_{(i, j)}^{\prime} \rightarrow v_{(j, k)}^{\prime} \rightarrow a_{k}^{\prime}}{b_{j}^{\prime} \rightarrow v_{(i, j)}^{\prime} \rightarrow v_{(i, k)}^{\prime} \rightarrow v_{(j, k)}^{\prime} \rightarrow a_{k}^{\prime}}$ & $\frac{(2,1,1)}{(2,0,1)}$ \\
\hline$(1,1,1)$ & $b_{j} \rightarrow v_{(j, k)} \rightarrow v_{(i, k)} \rightarrow v_{(i, j)} \rightarrow a_{j}$ & $b_{j}^{\prime} \rightarrow v_{(i, j)}^{\prime} \rightarrow v_{(i, k)}^{\prime} \rightarrow v_{(j, k)}^{\prime} \rightarrow a_{j}^{\prime}$ & $(1,0,1)$ \\
\hline$(1,1,1)$ & $b_{k} \rightarrow v_{(j, k)} \rightarrow v_{(i, k)} \rightarrow a_{k}$ & $b_{k}^{\prime} \rightarrow v_{(i, k)}^{\prime} \rightarrow v_{(j, k)}^{\prime} \rightarrow a_{k}^{\prime}$ & $(1,0,1)$ \\
\hline$(1,1,0)$ & $b_{k} \rightarrow v_{(j, k)} \rightarrow v_{(i, k)} \rightarrow v_{(i, j)} \rightarrow a_{j}$ \\
\hline$(1,0,0)$ & $b_{k} \rightarrow v_{(j, k)} \rightarrow v_{(i, j)} \rightarrow a_{j}$ & $b_{k}^{\prime} \rightarrow v_{(i, k)}^{\prime} \rightarrow v_{(j, k)}^{\prime} \rightarrow a_{j}^{\prime}$ & $(0,0,1)$ \\
\hline$(0,0,0)$ & $b_{k} \rightarrow v_{(j, k)} \rightarrow v_{(i, j)} \rightarrow a_{i}$ & $b_{k}^{\prime} \rightarrow v_{(i, k)}^{\prime} \rightarrow a_{i}^{\prime}$ & $(0,0,0)$ \\
\hline
\end{tabular}

TABLE 1. Shapes of paths locally around $F$ (resp. $\left.F^{\prime}\right)$ in $\mathcal{P}_{\underline{w}}\left(\operatorname{resp} . \mathcal{P}_{\mu_{F}(\underline{w})}\right)$ for orientation $\left(l_{r}, l_{r+1}\right)$ with $i<j<k \leq r$ (see Figure 10) and how they are mapped onto each other by $\operatorname{mut}_{F}$.

Definition 23. Let $\mathbf{p} \in \mathcal{P}_{\underline{w}}$ for $\underline{w} \in S_{n}$ and consider a mutable face $F$ of $\mathrm{pa}(\underline{w})$. Set $\delta_{F \subset A_{\mathbf{p}}}:=1$ if $F \in \mathrm{A}_{\mathbf{p}}$ and zero otherwise. Then we define the $F$-local type of $\mathbf{p}$ as the triple

$$
F(\mathbf{p}):=\left(i_{F, \mathbf{p}}, x_{F, \mathbf{p}}, o_{F, \mathbf{p}}\right):=\left(\#\left\{\operatorname{In}_{F} \cap \mathrm{A}_{\mathbf{p}}\right\}, \delta_{F \in \mathrm{A}_{\mathbf{p}}}, \#\left\{\mathrm{Out}_{F} \cap \mathrm{A}_{\mathbf{p}}\right\}\right) .
$$

For example, if $\mathrm{A}_{\mathbf{p}}$ in Figure 2 contains the faces $F, F_{\mathrm{in}_{1}}$ and $F_{\mathrm{out}_{2}}$ but not $F_{\mathrm{in}_{1}}$ and $F_{\text {out }_{1}}$, then the $F$-local type of $\mathbf{p}$ is $(1,1,1)$. The following lemma is a crucial observation on the $F$-local type of GP-paths.

Lemma 2. Let $\mathbf{p} \in \mathcal{P}_{\underline{w}}$ for $\underline{w} \in S_{n}$ and consider a mutable face $F$ of $\operatorname{pa}(\underline{w})$. Then the following are all possible $F$-local types $\mathbf{p}$ can have:

$i_{F, \mathbf{p}}=o_{F, \mathbf{p}}$ : then $F(\mathbf{p}) \in\{(0,0,0),(1,0,1),(1,1,1),(2,1,2)\}$;

$i_{F, \mathbf{p}}<o_{F, \mathbf{p}}:$ then $F(\mathbf{p}) \in\{(1,1,2),(0,0,1)\}$

$i_{F, \mathbf{p}}>o_{F, \mathbf{p}}$ : then $F(\mathbf{p}) \in\{(1,0,0),(1,1,0),(2,0,1),(2,1,1)\}$.

Moreover, the $F$-local types of $\mathbf{p}$ with $i_{F, \mathbf{p}}>o_{F, \mathbf{p}}$ come in pairs as $((1,0,0),(1,1,0))$ or $((2,0,1),(2,1,1))$. Meaning that if a path of one type exists for a fixed orientation then so does a path of the corresponding other type for the same orientation.

Proof. The lemma follows from case-by-case consideration of all possible shapes of $\mathbf{p} \in \mathcal{P}_{\underline{w}}$ around a mutable face $F$ of $\mathrm{pa}(\underline{w})$. First observe, that $F$ can have two different shapes, depending on whether it is defined by simple reflections $s_{m} s_{m+1} s_{m}$ (as on the left in Figure 2) or by $s_{m+1} s_{m} s_{m+1}$ (as on the right in Figure 2). We endow pa $(\underline{w})$ for either case of $F$ with all possible orientations $\left(l_{r}, l_{r+1}\right)$. Then locally at $F$, there are four cases of orientation depending on $r$ and $r+1$ in relation to $i, j, k$ (see Figure 10). We consider all possibilities for the path $\mathbf{p}$ to pass $F$ for each case of orientation and shape of $F$. These are listed in Tables 1 to 4 , in the second column for $F$ as on the left of Figure 10 and in the third for $F$ as on the right of Figure 10. In the first and last columns of these tables we indicate the corresponding $F$-local type. Observe, that the list in the claim of the lemma covers all occurring $F$-local types.

Regarding the second part of the claim, this also follows as an observation from Tables 1 to 4 . 


\begin{tabular}{|c|c|c|c|}
\hline$F$-local type of $\mathbf{p}$ & $\mathbf{p}$ in pa $(\underline{w})$ & $\mathbf{p}^{\prime}=\operatorname{mut}_{F}(\mathbf{p})$ in pa $\left(\mu_{F}(\underline{w})\right)$ & $F^{\prime}$-local type of $\mathbf{p}^{\prime}$ \\
\hline \hline$(1,0,1)$ & $b_{i} \rightarrow v_{(i, k)} \rightarrow v_{(j, k)} \rightarrow v_{(i, j)} \rightarrow a_{i}$ & $b_{i}^{\prime} \rightarrow v_{(i, j)}^{\prime} \rightarrow v_{(j, k)}^{\prime} \rightarrow v_{(i, k)}^{\prime} \rightarrow a_{i}^{\prime}$ & $(1,1,1)$ \\
\hline$\frac{(2,1,1)}{(2,0,1)}$ & $\frac{b_{i} \rightarrow v_{(i, k)} \rightarrow v_{(i, j)} \rightarrow a_{j}}{b_{i} \rightarrow v_{(i, k)} \rightarrow v_{(j, k)} \rightarrow v_{(i, j)} \rightarrow a_{j}}$ & $b_{i}^{\prime} \rightarrow v_{(i, j)}^{\prime} \rightarrow v_{(j, k)}^{\prime} \rightarrow a_{j}^{\prime}$ & $(1,1,2)$ \\
\hline$(1,0,1)$ & $b_{i} \rightarrow v_{(i, k)} \rightarrow v_{(j, k)} \rightarrow b_{k}$ & $b_{i}^{\prime} \rightarrow v_{(i, j)}^{\prime} \rightarrow v_{(j, k)}^{\prime} \rightarrow v_{(i, k)}^{\prime} \rightarrow b_{k}^{\prime}$ & $(1,1,1)$ \\
\hline$(0,0,1)$ & $b_{j} \rightarrow v_{(j, k)} \rightarrow v_{(i, j)} \rightarrow a_{i}$ & $b_{j}^{\prime} \rightarrow v_{(i, j)}^{\prime} \rightarrow v_{(j, k)}^{\prime} \rightarrow v_{(i, k)}^{\prime} \rightarrow a_{i}^{\prime}$ & $\frac{(1,1,0)}{(1,0,0)}$ \\
\hline$(1,0,1)$ & $b_{j} \rightarrow v_{(j, k)} \rightarrow v_{(i, j)} \rightarrow a_{j}$ & $b_{i}^{\prime} \rightarrow v_{(i, j)}^{\prime} \rightarrow v_{(i, k)}^{\prime} \rightarrow a_{i}^{\prime}$ & $(1,1,1)$ \\
\hline$(0,0,1)$ & $b_{j} \rightarrow v_{(j, k)} \rightarrow b_{k}$ & $\frac{b_{j}^{\prime} \rightarrow v_{(i, j)}^{\prime} \rightarrow v_{(j, k)}^{\prime} \rightarrow v_{(j, k)}^{\prime} \rightarrow a_{j}^{\prime}}{(i, k)} \rightarrow b_{k}^{\prime}$ \\
\hline$(1,0,1)$ & $a_{k} \rightarrow v_{(i, k)} \rightarrow v_{(j, k)} \rightarrow v_{(i, j)} \rightarrow a_{i}$ & $a_{j}^{\prime} \rightarrow v_{(i, j)}^{\prime} \rightarrow v_{(i, k)}^{\prime} \rightarrow b_{k}^{\prime}$ & $(1,1,0)$ \\
\hline$(2,1,1)$ & $a_{k} \rightarrow v_{(i, k)}^{\prime} \rightarrow v_{(i, j)} \rightarrow a_{j}$ & $a_{i}^{\prime}$ & $(1,0,0)$ \\
\hline$(2,0,1)$ & $a_{k} \rightarrow v_{(i, k)} \rightarrow v_{(j, k)} \rightarrow v_{(i, j)} \rightarrow a_{j}$ & $a_{k}^{\prime} \rightarrow v_{(j, k)}^{\prime} \rightarrow a_{j}^{\prime}$ & $(1,1,2)$ \\
\hline$(1,0,1)$ & $a_{k} \rightarrow v_{(i, k)} \rightarrow v_{(j, k)} \rightarrow b_{k}$ & $a_{k}^{\prime} \rightarrow v_{(j, k)}^{\prime} \rightarrow v_{(i, k)}^{\prime} \rightarrow b_{k}^{\prime}$ & $(1,1,1)$ \\
\hline
\end{tabular}

TABLE 2. Shapes of paths locally around $F$ (resp. $\left.F^{\prime}\right)$ in $\mathcal{P}_{\underline{w}}\left(\operatorname{resp} . \mathcal{P}_{\mu_{F}(\underline{w})}\right)$ for orientation $\left(l_{r}, l_{r+1}\right)$ with $i<j \leq r$ and $r+1 \leq k$ (see Figure 10) and how they are mapped onto each other by $\operatorname{mut}_{F}$.

With notation as in the lemma, if $\mathbf{p}_{1}, \mathbf{p}_{2}$ are paths with $i_{F, \mathbf{p}_{j}}>o_{F, \mathbf{p}_{j}}, j=1,2$ such that $\left(\left(i_{F, \mathbf{p}_{1}}, x_{F, \mathbf{p}_{1}}, o_{F, \mathbf{p}_{1}}\right),\left(i_{F, \mathbf{p}_{2}}, x_{F, \mathbf{p}_{2}}, o_{F, \mathbf{p}_{2}}\right)\right)$ is one of the pairs, then we denote by $\mathbf{p}_{1} \oplus \mathbf{p}_{2}$ their formal sum. If $\mathbf{p}_{1}$ and $\mathbf{p}_{2}$ are equal away from $F$, we denote this by $\mathbf{p}_{1} / F=\mathbf{p}_{2} / F$. Observe, that this is the case here. With this notation we define the following set of paths, respectively formal sums of paths.

$$
\widetilde{\mathcal{P}}_{\underline{w}, F}:=\left\{\begin{array}{c|c}
\mathbf{p}, & \mathbf{p} \in \mathcal{P}_{\underline{w}} \text { with } i_{F, \mathbf{p}}=o_{F, \mathbf{p}} \text { or } i_{F, \mathbf{p}}<o_{F, \mathbf{p}}, \\
\mathbf{p}_{1} \oplus \mathbf{p}_{2} & \mathbf{p}_{1}, \mathbf{p}_{2} \in \mathcal{P}_{\underline{w}} \text { with } i_{F, \mathbf{p}_{j}}>o_{F, \mathbf{p}_{j}} \text { for } j=1,2
\end{array}\right\} .
$$

Note that for every mutable face $F$ of pa $(\underline{w})$ every path in $\mathcal{P}_{\underline{w}}$ appears in $\widetilde{\mathcal{P}}_{\underline{w}, F}$ either on its own or as a formal summand. This additional structure on $\mathcal{P}_{\underline{w}}$ allows us to define mutation on it.

Definition 24. Let $w \in S_{n}$ with reduced expressions $\underline{w}$ and $\mu_{F}(\underline{w})$, where $F$ is a mutable face in $\mathrm{pa}(\underline{w})$. Denote by $F^{\prime}$ the corresponding face in $\operatorname{pa}\left(\mu_{F}(\underline{w})\right)$. We define $\operatorname{mut}_{F}: \widetilde{\mathcal{P}}_{\underline{w}, F} \rightarrow$ $\widetilde{\mathcal{P}}_{\mu_{F}(\underline{w}), F^{\prime}}$ depending on the $F$-local type by

$i_{F, \mathbf{p}}=o_{F, \mathbf{p}}: \operatorname{mut}_{F}(\mathbf{p})=\mathbf{p}^{\prime}$ with $\mathbf{p} / F=\mathbf{p}^{\prime} / F^{\prime}$, where for $F(\mathbf{p}) \in\{(0,0,0),(2,1,2)\}$ we have $F(\mathbf{p})=$ $F^{\prime}\left(\mathbf{p}^{\prime}\right)$, and for $F(\mathbf{p}) \in\{(1,0,1),(1,1,1)\}$ we have $F^{\prime}\left(\mathbf{p}^{\prime}\right)=\left(i_{F, \mathbf{p}},\left|x_{F, \mathbf{p}}-1\right|, o_{F, \mathbf{p}}\right) ;$

$i_{F, \mathbf{p}}<o_{F, \mathbf{p}}: \operatorname{mut}_{F}(\mathbf{p})=\mathbf{p}_{1}^{\prime} \oplus \mathbf{p}_{2}^{\prime}$ with $\mathbf{p} / F=\mathbf{p}_{1}^{\prime} / F^{\prime}=\mathbf{p}_{2}^{\prime} / F^{\prime}$, for $F(\mathbf{p}) \in\{(0,0,1),(1,1,2)\}$ with $F^{\prime}\left(\mathbf{p}_{1}^{\prime}\right)=\left(o_{F, \mathbf{p}}, x_{F, \mathbf{p}}, i_{F, \mathbf{p}}\right)$ and $F^{\prime}\left(\mathbf{p}_{2}^{\prime}\right)=\left(o_{F, \mathbf{p}},\left|x_{F, \mathbf{p}}-1\right|, i_{F, \mathbf{p}}\right) ;$

$i_{F, \mathbf{p}}>o_{F, \mathbf{p}}: \operatorname{mut}_{F}\left(\mathbf{p}_{1} \oplus \mathbf{p}_{2}\right)=\mathbf{p}^{\prime}$ with $\mathbf{p}_{1} / F=\mathbf{p}_{2} / F=\mathbf{p}^{\prime} / F^{\prime}$, for $\left(F\left(\mathbf{p}_{1}\right), F\left(\mathbf{p}_{2}\right)\right)$ either being $((1,0,0),(1,1,0))$ or $((2,1,1),(2,0,1))$ with $F^{\prime}\left(\mathbf{p}^{\prime}\right)=\left(o_{F, \mathbf{p}_{1}}, x_{F, \mathbf{p}_{1}}, i_{F, \mathbf{p}_{1}}\right)$.

Consider the torus $\mathcal{X}_{\underline{w}}$ corresponding to the seed (associated with) pa $(\underline{w})$. For the lattice $N_{\underline{w}}$ we have the basis $\left\{\bar{e}_{E}\right\}_{E}$ face of pa( $\left.\underline{w}\right)$. Then $e_{\mathbf{p}} \in N$ for $\mathbf{p} \in \mathcal{P}_{\underline{w}, F}$ is an expression in this 


\begin{tabular}{|c|c|c|c|}
\hline$F$-local type of $\mathbf{p}$ & $\mathbf{p}$ in $\mathrm{pa}(\underline{w})$ & $\mathbf{p}^{\prime}=\operatorname{mut}_{F}(\mathbf{p})$ in $\mathrm{pa}\left(\mu_{F}(\underline{w})\right)$ & $F^{\prime}$-local type of $\mathbf{p}^{\prime}$ \\
\hline$(1,1,1)$ & $b_{i} \rightarrow v_{(i, k)} \rightarrow v_{(i, j)} \rightarrow a_{i}$ & $b_{i}^{\prime} \rightarrow v_{(i, j)}^{\prime} \rightarrow v_{(i, k)}^{\prime} \rightarrow a_{i}^{\prime}$ & $(1,0,1)$ \\
\hline$(1,0,0)$ & $b_{i} \rightarrow v_{(i, k)} \rightarrow v_{(j, k)} \rightarrow b_{j}$ & \multirow{2}{*}{$b_{i}^{\prime} \rightarrow v_{(i, j)}^{\prime} \rightarrow b_{k}$} & \multirow{2}{*}{$(0,0,1)$} \\
\hline$(1,1,0)$ & $b_{i} \rightarrow v_{(i, k)} \rightarrow v_{(i, j)} \rightarrow v_{(j, k)} \rightarrow b_{j}$ & & \\
\hline$(1,1,1)$ & $b_{i} \rightarrow v_{(i, k)} \rightarrow v_{(i, j)} \rightarrow v_{(j, k)} \rightarrow b_{k}$ & $b_{i}^{\prime} \rightarrow v_{(i, j)}^{\prime} \rightarrow v_{(i, k)}^{\prime} \rightarrow b_{k}^{\prime}$ & $(1,0,1)$ \\
\hline \multirow{2}{*}{$(1,1,2)$} & \multirow{2}{*}{$a_{j} \rightarrow v_{(i, j)} \rightarrow a_{i}$} & \multirow{2}{*}{$\begin{array}{c}a_{j}^{\prime} \rightarrow v_{(j, k)}^{\prime} \rightarrow v_{(i, k)}^{\prime} \rightarrow a_{i}^{\prime} \\
a_{j}^{\prime} \rightarrow v_{(j, k)}^{\prime} \rightarrow v_{(i, j)}^{\prime} \rightarrow v_{(i, k)}^{\prime} \rightarrow a_{i}^{\prime}\end{array}$} & $(2,1,1)$ \\
\hline & & & $(2,0,1)$ \\
\hline$(1,1,1)$ & $a_{j} \rightarrow v_{(i, j)} \rightarrow v_{(j, k)} \rightarrow b_{j}$ & $a_{j}^{\prime} \rightarrow v_{(j, k)}^{\prime} \rightarrow v_{(i, j)}^{\prime} \rightarrow b_{j}^{\prime}$ & $(1,0,1)$ \\
\hline \multirow{2}{*}{$(1,1,2)$} & \multirow{2}{*}{$a_{j} \rightarrow v_{(i, j)} \rightarrow v_{(j, k)} \rightarrow b_{k}$} & \multirow{2}{*}{$\begin{array}{c}\quad a_{j}^{\prime} \rightarrow v_{(j, k)}^{\prime} \rightarrow v_{(i, k)}^{\prime} \rightarrow b_{k}^{\prime} \\
a_{j}^{\prime} \rightarrow v_{(j, k)}^{\prime} \rightarrow v_{(i, j)}^{\prime} \rightarrow v_{(i, k)}^{\prime} \rightarrow b_{k}^{\prime}\end{array}$} & $(2,1,1)$ \\
\hline & & & $(2,0,1)$ \\
\hline$(1,1,1)$ & $a_{k} \rightarrow v_{(i, k)} \rightarrow v_{(i, j)} \rightarrow a_{i}$ & $a_{k}^{\prime} \rightarrow v_{(j, k)}^{\prime} \rightarrow v_{(i, j)}^{\prime} \rightarrow v_{(i, k)}^{\prime} \rightarrow a_{i}^{\prime}$ & $(1,0,1)$ \\
\hline$(1,0,0)$ & $a_{k} \rightarrow v_{(i, k)} \rightarrow v_{(j, k)} \rightarrow b_{j}$ & \multirow{2}{*}{$a_{k}^{\prime} \rightarrow v_{(j, k)}^{\prime} \rightarrow v_{(i, j)}^{\prime} \rightarrow b_{j}^{\prime}$} & \multirow{2}{*}{$(0,0,1)$} \\
\hline$(1,1,0)$ & $a_{k} \rightarrow v_{(i, k)} \rightarrow v_{(i, j)} \rightarrow v_{(j, k)} \rightarrow b_{j}$ & & \\
\hline$(1,1,1)$ & $a_{k} \rightarrow v_{(i, k)} \rightarrow v_{(i, j)} \rightarrow v_{(j, k)} \rightarrow b_{k}$ & $a_{k}^{\prime} \rightarrow v_{(j, k)}^{\prime} \rightarrow v_{(i, j)}^{\prime} \rightarrow v_{(i, k)}^{\prime} \rightarrow b_{k}^{\prime}$ & $(1,0,1)$ \\
\hline
\end{tabular}

TABLE 3. Shapes of paths locally around $F$ (resp. $\left.F^{\prime}\right)$ in $\mathcal{P}_{\underline{w}}\left(\operatorname{resp} . \mathcal{P}_{\mu_{F}(\underline{w})}\right)$ for orientation $\left(l_{r}, l_{r+1}\right)$ with $i \leq r$ and $r+1 \leq j<k$ (see Figure 10) and how they are mapped onto each other by $\operatorname{mut}_{F}$.

basis and $z^{e_{\mathbf{p}}}$ a function on $\mathcal{X}_{\underline{w}}$. To extend our definition of $e_{\mathbf{p}}$ in (3.6) for $\mathbf{p} \in \mathcal{P}_{\underline{w}}$ to $\mathbf{p} \in \widetilde{\mathcal{P}}_{\underline{w}, F}$, we set $z^{e_{\mathbf{p}_{1} \oplus \mathbf{p}_{2}}}:=z^{e_{\mathbf{p}_{1}}}+z^{e_{\mathbf{p}_{2}}}$. Then for every mutable face $F$ of pa $(\underline{w})$ we have

$$
\left\{\begin{array}{l|l}
\mathbf{x} \in \mathbb{R}^{\ell(w)} \mid\left(\sum_{\mathbf{p} \in \widetilde{\mathcal{P}}_{\underline{w}, F}} z^{e_{\mathbf{p}}}\right)^{\operatorname{trop}}(\mathbf{x}) \geq 0
\end{array}\right\}=S_{\underline{w}} .
$$

The following is the key lemma of this section.

Lemma 3. Let $w \in S_{n}$ with reduced expressions $\underline{w}$ and $\mu_{F}(\underline{w})$, where $F$ is a mutable face of $\mathrm{pa}(\underline{w})$ and $F^{\prime}$ the corresponding face of $\mathrm{pa}\left(\mu_{F}(\underline{w})\right)$ (i.e. $\left.\mu_{F^{\prime}}\left(\mu_{F}(\underline{w})\right)=\underline{w}\right)$. Let $\left\{e_{E}\right\}_{E}$ denote the basis for $N_{\underline{w}}$ and $\left\{e_{E}^{\prime}\right\}_{E}$ the basis for $N_{\mu_{F}(\underline{w})}$. Then for $\mathbf{p} \in \widetilde{\mathcal{P}}_{\underline{w}, F}$ we have

$$
\mu_{F^{\prime}}^{*}\left(z^{e_{\mathbf{p}}}\right)=z^{e_{\text {mut }_{F}(\mathbf{p})}^{\prime}} \text {. }
$$

Proof. We prove the claim case-by-case depending on the $F$-local type of $\mathbf{p}$ as in Lemma 2. As notation we use $n \in N_{\underline{w}}$ (resp. $n^{\prime} \in N_{\mu_{F}(\underline{w})}$ ) referring to an expression of $n$ is the basis $\left\{e_{E}\right\}_{E \text { face of } \mathrm{pa}(\underline{w})}$ (resp. $\left\{e_{E}^{\prime}\right\}_{E}$ face of $\operatorname{pa}\left(\mu_{F}(\underline{w})\right)$ ). Consider $\mathbf{p} \in \mathcal{P}_{\underline{w}}$, then

$$
e_{\mathbf{p}}=-\sum_{E \subset \mathrm{A}_{\mathbf{p}}} e_{E}=-\sum_{E \subset\left(\operatorname{In}_{F} \cup \mathrm{Out}_{F}\right) \cap \mathrm{A}_{\mathbf{p}}} e_{E}-\sum_{E \not \subset\left(\operatorname{In}_{F} \cup \mathrm{Out}_{F}\right) \cap \mathrm{A}_{\mathbf{p}}} e_{E}=: n_{\mathbf{p}}+m_{\mathbf{p}}
$$

As by definition $\operatorname{mut}_{F}$ effects a path only locally around $F$, we have $m_{\mathbf{p}}=m_{\text {mut }_{F}(\mathbf{p})}$ (resp. $m_{\mathbf{p}}=m_{\mathbf{p}_{1}^{\prime}}=m_{\mathbf{p}_{2}^{\prime}}$ if $\left.\operatorname{mut}_{F}(\mathbf{p})=\mathbf{p}_{1}^{\prime} \oplus \mathbf{p}_{2}^{\prime} \in \widetilde{\mathcal{P}}_{\mu_{F}(\underline{w}), F^{\prime}}\right)$. Both have the same expressions in bases $\left\{e_{E}\right\}_{E}$ and $\left\{e_{E}^{\prime}\right\}_{E}$ as the corresponding basis elements are not effected by mutation: only basis elements corresponding to vertices (i.e. faces of $\mathrm{pa}(\underline{w})$ ) adjacent to $F$ (i.e. in $\operatorname{In}_{F} \cup$ Out $\left._{F}\right)$ are changed by mutation in (2.2). We use this fact throughout the proof. Denote 


\begin{tabular}{|c|c|c|c|}
\hline$F$-local type of $\mathbf{p}$ & $\mathbf{p}$ in pa $(\underline{w})$ & $\mathbf{p}^{\prime}=\operatorname{mut}_{F}(\mathbf{p})$ in pa $\left(\mu_{F}(\underline{w})\right)$ & $F^{\prime}$-local type of $\mathbf{p}^{\prime}$ \\
\hline \hline$(1,0,1)$ & $a_{i} \rightarrow v_{(i, j)} \rightarrow v_{(i, k)} \rightarrow b_{i}$ & $a_{i}^{\prime} \rightarrow v_{(i, k)}^{\prime} \rightarrow v_{(i, j)}^{\prime} \rightarrow b_{i}^{\prime}$ & $(1,1,1)$ \\
\hline$\frac{(2,0,1)}{(2,1,1)}$ & $\begin{array}{c}a_{i} \rightarrow v_{(i, j)} \rightarrow v_{(i, k)} \rightarrow v_{(j, k)} \rightarrow b_{j} \\
a_{i} \rightarrow v_{(i, j)} \rightarrow v_{(j, k)} \rightarrow b_{j}\end{array}$ & $a_{i}^{\prime} \rightarrow v_{(i, k)}^{\prime} \rightarrow v_{(i, j)}^{\prime} \rightarrow b_{j}^{\prime}$ & $(1,1,2)$ \\
\hline$(2,1,2)$ & $a_{i} \rightarrow v_{(i, j)} \rightarrow v_{(j, k)} \rightarrow b_{k}$ & $a_{i}^{\prime} \rightarrow v_{(i, k)}^{\prime} \rightarrow b_{k}^{\prime}$ & $(2,1,2)$ \\
\hline$(1,0,1)$ & $a_{j} \rightarrow v_{(i, j)} \rightarrow v_{(i, k)} \rightarrow v_{(j, k)} \rightarrow b_{j}$ & $a_{j}^{\prime} \rightarrow v_{(j, k)}^{\prime} \rightarrow v_{(i, k)}^{\prime} \rightarrow v_{(i, j)}^{\prime} \rightarrow b_{j}^{\prime}$ & $(1,1,1)$ \\
\hline$(0,0,0)$ & $a_{k} \rightarrow v_{(i, k)} \rightarrow b_{i}$ & $a_{k}^{\prime} \rightarrow v_{(j, k)}^{\prime} \rightarrow v_{(i, j)}^{\prime} \rightarrow b_{i}^{\prime}$ & $(0,0,0)$ \\
\hline$(0,0,1)$ & $a_{j} \rightarrow v_{(i, j)} \rightarrow v_{(i, k)} \rightarrow b_{i}$ & $a_{j}^{\prime} \rightarrow v_{(j, k)}^{\prime} \rightarrow v_{(i, j)}^{\prime} \rightarrow b_{i}^{\prime}$ & $(1,0,0)$ \\
\hline
\end{tabular}

TABLE 4. Shapes of paths locally around $F$ (resp. $\left.F^{\prime}\right)$ in $\mathcal{P}_{\underline{w}}\left(\operatorname{resp} . \mathcal{P}_{\mu_{F}(\underline{w})}\right)$ for orientation $\left(l_{r}, l_{r+1}\right)$ with $r+1 \leq i<j<k$ (see Figure 10) and how they are mapped onto each other by mut $_{F}$.

basis elements associated with faces $F_{\mathrm{in}}, F_{\mathrm{in}_{1}}, F_{\mathrm{in}_{2}} \in \mathrm{In}_{F}$ by $e_{\mathrm{in}}, e_{\mathrm{in}_{1}}, e_{\mathrm{in}_{2}}$ and similarly for $e_{\mathrm{out}}$. After mutation, $e_{\text {in }}^{\prime}$ is associated with the face $F_{\text {in }}^{\prime} \in \operatorname{Out}_{F^{\prime}}$ in $\operatorname{pa}\left(\mu_{F}(\underline{w})\right)$.

We distinguish the cases as in Lemma 2.

$i_{F, \mathbf{p}}<o_{F, \mathbf{p}}$ From Lemma 2 we know that in this case $n_{\mathbf{p}}=-e_{\mathrm{in}}-e_{F}-e_{\text {out }_{1}}-e_{\text {out }_{2}}$ (resp. $\left.n_{\mathbf{p}}=-e_{\text {out }}\right)$ and $\operatorname{mut}_{F}(\mathbf{p})=\mathbf{p}_{1}^{\prime} \oplus \mathbf{p}_{2}^{\prime}$ with $\mathbf{p}_{1}^{\prime}, \mathbf{p}_{2}^{\prime}$ as in Definition 24. Then $n_{\mathbf{p}_{1}^{\prime}}^{\prime}=$ $-e_{\text {in }}^{\prime}-e_{F}^{\prime}-e_{\text {out }_{1}}^{\prime}-e_{\text {out }_{2}}^{\prime}$ (resp. $\left.n_{\mathbf{p}_{1}^{\prime}}^{\prime}=-e_{\text {out }}^{\prime}-e_{F}\right)$ and $n_{\mathbf{p}_{2}^{\prime}}^{\prime}=-e_{\text {in }_{1}}^{\prime}-e_{\text {out }_{1}}^{\prime}-e_{\text {out }_{2}}^{\prime}$ (resp. $n_{\mathbf{p}_{1}^{\prime}}^{\prime}=-e_{\text {out }}^{\prime}$ ). We compute using formulas $(2.2),(2.3)$ and the observation that $m_{\mathbf{p}_{1}^{\prime}}^{\prime}=m_{\mathbf{p}_{2}^{\prime}}^{\prime}:$

$$
\begin{aligned}
& \mu_{F^{\prime}}^{*}\left(z^{n_{\mathbf{p}}+m_{\mathbf{p}}}\right)=z^{-e_{\mathrm{in}}-e_{F}-e_{\mathrm{out}_{1}}-e_{\mathrm{out}_{2}}+m_{\mathbf{p}}}\left(1+z^{e_{F}}\right) \\
& =z^{-e_{\mathrm{in}^{\prime}}^{\prime}-e_{\mathrm{out}_{1}}^{\prime}-e_{\mathrm{out}_{2}}^{\prime}+m_{\mathbf{p}^{\prime}}^{\prime}\left(1+z^{-e_{F}^{\prime}}\right)}
\end{aligned}
$$

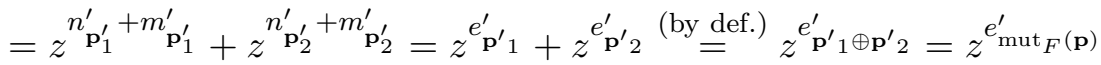

$$
\begin{aligned}
& \text { (resp. } \mu_{F^{\prime}}^{*}\left(z^{n_{\mathbf{p}}+m_{\mathbf{p}}}\right)=z^{-e_{\mathrm{out}}+m_{\mathbf{p}}}\left(1+z^{e_{F}}\right)=z^{-e_{\mathrm{out}}^{\prime}+m_{\mathbf{p}^{\prime}}^{\prime}}\left(1+z^{-e_{F}^{\prime}}\right)
\end{aligned}
$$

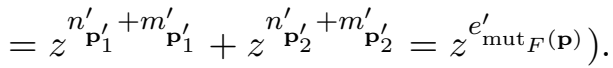

$i_{F, \mathbf{p}}=o_{F, \mathbf{p}}$ In this case $\operatorname{mut}_{F}(\mathbf{p})=\mathbf{p}^{\prime} \in \widetilde{\mathcal{P}}_{\mu_{F}(\underline{w}), F^{\prime}}$ as in Definition 24 . We divide into three cases: $i_{F, \mathbf{p}} \in\{0,1,2\}$. If $i_{F, \mathbf{p}}=0$, consider $\mathrm{A}_{\mathbf{p}}=F_{1} \cup \cdots \cup F_{r}$ then $\mathrm{A}_{\mathbf{p}^{\prime}}=F_{1}^{\prime} \cup \cdots \cup F_{r}^{\prime}$. Further,

$$
\mu_{F^{\prime}}^{*}\left(z^{e_{\mathbf{p}}}\right)=\mu_{F^{\prime}}^{*}\left(z^{m_{\mathbf{p}}}\right)=z^{m_{\mathbf{p}}}=z^{m_{\mathbf{p}^{\prime}}}=z^{e_{\mathbf{p}^{\prime}}^{\prime}}=z^{e_{\operatorname{mut}_{F}(\mathbf{p})}^{\prime}}
$$

If $i_{F, \mathbf{p}}=1$ we have $n_{\mathbf{p}}=-e_{F}-e_{\text {in }}-e_{\text {out }}$ (resp. $\left.n_{\mathbf{p}}=-e_{\text {in }}-e_{\text {out }}\right)$. We have $n_{\mathbf{p}^{\prime}}^{\prime}=-e_{\text {in }}^{\prime}-e_{\text {out }}^{\prime}$ (resp. $\left.n_{\mathbf{p}^{\prime}}^{\prime}=-e_{F}^{\prime}-e_{\text {in }}^{\prime}-e_{\text {out }}^{\prime}\right)$ and compute

$$
\mu_{F^{\prime}}^{*}\left(z^{n_{\mathbf{p}}}\right)=z^{-e_{F}-e_{\mathrm{in}}-e_{\mathrm{out}}}=z^{e_{F}^{\prime}-\left(e_{\mathrm{in}}^{\prime}+e_{F}^{\prime}\right)-e_{\mathrm{out}}^{\prime}}=z^{-e_{\mathrm{in}}^{\prime}-e_{\mathrm{out}}^{\prime}}=z^{n_{\mathbf{p}^{\prime}}^{\prime}}
$$

(resp. $\left.\mu_{F^{\prime}}^{*}\left(z^{n_{\mathbf{p}}}\right)=z^{-e_{\mathrm{in}}-e_{\mathrm{out}}}=z^{-\left(e_{\mathrm{in}}^{\prime}+e_{F}^{\prime}\right)-e_{\mathrm{out}}^{\prime}}=z^{-e_{F}^{\prime}-e_{\mathrm{in}}^{\prime}-e_{\mathrm{out}}^{\prime}}=z^{n_{\mathbf{p}^{\prime}}^{\prime}}\right)$. 
If $i_{F, \mathbf{p}}=2$ we have $n_{\mathbf{p}}=-e_{\mathrm{in}_{1}}-e_{\mathrm{in}_{2}}-e_{F}-e_{\mathrm{out}_{1}}-e_{\mathrm{out}_{2}}$. Now $n_{\mathbf{p}^{\prime}}^{\prime}=-e_{\mathrm{in}_{1}}^{\prime}-e_{\mathrm{in}_{2}}^{\prime}-$ $e_{F}^{\prime}-e_{\text {out }_{1}}^{\prime}-e_{\text {out }_{2}}^{\prime}$ and we compute

$$
\begin{aligned}
\mu_{F^{\prime}}^{*}\left(z^{n_{\mathbf{p}}}\right) & =z^{-e_{\mathrm{in}_{1}}-e_{\mathrm{in}_{2}}-e_{F}-e_{\mathrm{out}_{1}}-e_{\mathrm{out}_{2}}}=z^{-\left(e_{\mathrm{in}_{1}}^{\prime}+e_{F}^{\prime}\right)-\left(e_{\mathrm{in}_{2}}^{\prime}+e_{F}^{\prime}\right)-\left(-e_{F}^{\prime}\right)-e_{\mathrm{out}_{1}}^{\prime}-e_{\mathrm{out}_{2}}^{\prime}} \\
& =z^{-e_{\mathrm{in}_{1}}^{\prime}-e_{\mathrm{in}_{2}}^{\prime}-e_{F}^{\prime}-e_{\mathrm{out}_{1}}^{\prime}-e_{\mathrm{out}_{2}}^{\prime}}=z^{n_{\mathbf{p}^{\prime}}^{\prime}} .
\end{aligned}
$$

In all three cases the claim follows from the computation.

$i_{F, \mathbf{p}}>o_{F, \mathbf{p}}$ In this case by Lemma 2 there are paths $\mathbf{p}_{1}, \mathbf{p}_{2} \in \mathcal{P}_{\underline{w}}$ with $\mathbf{p}_{1} \oplus \mathbf{p}_{2} \in \widetilde{\mathcal{P}}_{\underline{w}, F}$ and $\operatorname{mut}_{F}\left(\mathbf{p}_{1} \oplus \mathbf{p}_{2}\right)=\mathbf{p}^{\prime} \in \widetilde{\mathcal{P}}_{\mu_{F}(\underline{w}), F^{\prime}}$ as in Definition 24 . We have $n_{\mathbf{p}_{1}}=-e_{\text {in }}$ and $n_{\mathbf{p}_{2}}=-e_{\mathrm{in}}-e_{F}$ (resp. $n_{\mathbf{p}_{1}}=-e_{\mathrm{in}_{1}}-e_{\mathrm{in}_{2}}-e_{\mathrm{out}}$ and $\left.n_{\mathbf{p}_{2}}=-e_{\mathrm{in}_{1}}-e_{\mathrm{in}_{2}}-e_{F}-e_{\mathrm{out}}\right)$. For $\mathbf{p}^{\prime}$ we have $n_{\mathbf{p}^{\prime}}^{\prime}=-e_{\mathrm{in}}^{\prime}\left(\right.$ resp. $\left.n_{\mathbf{p}^{\prime}}^{\prime}=-e_{\mathrm{in}_{1}}^{\prime}-e_{\mathrm{in} 2}^{\prime}-e_{F}^{\prime}-e_{\text {out }}^{\prime}\right)$. We compute

$$
\begin{aligned}
\mu_{F^{\prime}}^{*}\left(z^{n_{\mathbf{p}_{1}}}+z^{n_{\mathbf{p}_{2}}}\right) & =z^{-e_{\mathrm{in}}}\left(1+z^{e_{F}}\right)^{-1}+z^{-e_{\mathrm{in}}-e_{F}}\left(1+z^{e_{F}}\right)^{-1} \\
& =\left(z^{-e_{\mathrm{in}}^{\prime}-e_{F}^{\prime}}+z^{-e_{\mathrm{in}}^{\prime}}\right)\left(1+z^{-e_{F}^{\prime}}\right)^{-1}=z^{-e_{\mathrm{in}}^{\prime}}=z^{n_{\mathbf{p}^{\prime}}^{\prime}} \\
\text { (resp. } \mu_{F^{\prime}}^{*}\left(z^{n_{\mathbf{p}_{1}}}+z^{n_{\mathbf{p}_{2}}}\right) & =z^{-e_{\mathrm{in}_{1}}-e_{\mathrm{in}_{2}}-e_{\mathrm{out}}}\left(1+z^{e_{F}}\right)^{-1}+z^{-e_{\mathrm{in}_{1}}-e_{\mathrm{in}_{2}}-e_{F}-e_{\mathrm{out}}}\left(1+z^{e_{F}}\right)^{-1} \\
& =\left(z^{-e_{\mathrm{in}_{1}}^{\prime}-e_{\mathrm{in}_{2}}^{\prime}-2 e_{F}^{\prime}-e_{\mathrm{out}}^{\prime}}+z^{-e_{\mathrm{in}_{1}}^{\prime}-e_{\mathrm{in}_{2}}^{\prime}-e_{F}^{\prime}-e_{\mathrm{out}}^{\prime}}\right)\left(1+z^{-e_{F}^{\prime}}\right)^{-1} \\
& \left.=z^{-e_{\mathrm{in}_{1}}^{\prime}-e_{\mathrm{in}_{2}}^{\prime}-e_{F}^{\prime}-e_{\mathrm{out}}^{\prime}}=z^{n_{\mathbf{p}^{\prime}}^{\prime}}\right) .
\end{aligned}
$$

In both cases the claim follows.

Before proving a generalization of Proposition 8 we have to show that the normal vectors associated to the weight inequalities $e_{[i: k]}(3.8)$ mutate as expected. We use the notation as in Lemma 3 and its proof. Recall the normal vectors of the weight inequalities for $\mathcal{S}_{\underline{w}}$ from (3.8). For $i \in[n-1]$ let $e_{[i: 0]}, \ldots, e_{\left[i: n_{i}\right]}$ be those for $\underline{w}$ as expressions in $\left\{e_{E}\right\}_{E}$ and $e_{[i: 0]}^{\prime}, \ldots, e_{\left[i, n_{i}^{\prime}\right]}^{\prime}$ those for $\mu_{F}(\underline{w})$ as expressions in $\left\{e_{E}^{\prime}\right\}_{E}$.

Lemma 4. With notation as above we have for every $i \in[n-1]$

$$
\mu_{F^{\prime}}^{*}\left(\sum_{k=0}^{n_{i}} z^{e^{[i: k]}}\right)=\sum_{k^{\prime}=0}^{n_{i}^{\prime}} z^{e^{\prime}}{ }^{\left.i: k^{\prime}\right]} .
$$

Proof. We treat the case where $F$ is of level $l$ and $F^{\prime}$ of level $l+1$, with $l \in[n-2]$ (the proof of the other case is similar). Recall that $e_{[i: k]}=-e_{F_{i}}-e_{F_{j_{1}}}-\cdots-e_{F_{j_{k}}}$, where $k \in\left[0, n_{i}\right]$, $s_{j_{1}}, \ldots, s_{j_{n_{i}}}=s_{i}$ in $\underline{w}$, and $F_{j_{k}}$ is bounded to the left by the crossing in pa $(\underline{w})$ induced by $s_{j_{k}}$. Let $F_{i}^{\prime}, F_{j_{1}}^{\prime}, \ldots, F_{j_{n_{i}^{\prime}}^{\prime}}^{\prime}$ be the corresponding faces in pa $\left(\mu_{F}(\underline{w})\right)$. In particular, if $i \notin\{l, l+1\}$ we have

$$
\mu_{F^{\prime}}^{*}\left(z^{-e_{F_{i}}-e_{F_{j_{1}}}-\cdots-e_{F_{j_{k}}}}\right)=z^{-e_{F_{i}^{\prime}}^{\prime}-e_{F_{j_{1}}^{\prime}}^{\prime}-\cdots-e_{F_{j_{k}}^{\prime}}^{\prime}} .
$$

We therefore focus on the cases $i \in\{l, l+1\}$.

$i=l$ As $F$ is of level $l$ we have $F=F_{j_{k}}$ for one $k \in\left[n_{l}\right], s_{j_{k}}=s_{l}$. By (2.2) we have $\mu_{F}\left(e_{F_{l}}\right)=e_{F_{l}^{\prime}}^{\prime}$ and $\mu_{F}\left(e_{F_{j_{r}}}\right)=e_{F_{j_{r}}^{\prime}}^{\prime}$ for $r \in[k-1]$, hence

$$
\mu_{F^{\prime}}^{*}\left(z^{-e_{F_{l}}-e_{F_{j_{1}}}-\cdots-e_{F_{j_{r}}}}\right)=z^{-e_{F_{l}^{\prime}}^{\prime}-e_{F_{j_{1}}^{\prime}}^{\prime}-\cdots-e_{F_{j_{r}}^{\prime}}^{\prime}} .
$$


Still by $(2.2)$ we have $\mu_{F}\left(e_{F_{k-1}}\right)=e_{F_{j_{k-1}}^{\prime}}^{\prime}+e_{F_{j_{k}}^{\prime}}^{\prime}, \mu_{F}\left(e_{F_{j_{k}}}\right)=-e_{F_{j_{k}}^{\prime}}^{\prime}$ and $\mu_{F}\left(e_{F_{j_{s}}}\right)=e_{F_{j_{s}}^{\prime}}^{\prime}$ for $s \in\left[k+1, n_{l}\right]$. Plugging in to $(2.3)$ we obtain

$$
\begin{aligned}
& \mu_{F^{\prime}}^{*}\left(z^{e_{[l: k-1]}}+z^{e[l: k]}+z^{e_{[l: k+1]}}\right)=z^{-e_{F_{l}}-\cdots-e_{F_{j_{k-1}}}}\left(1+z^{e_{F_{j_{k}}}}\right)^{-1} \\
& +z^{-e_{F_{l}}-\cdots-e_{F_{j_{k}}}}\left(1+z^{\left.e_{F_{j_{k}}}\right)^{-1}}+z^{-e_{F_{l}}-\cdots-e_{F_{j_{k+1}}}}\right. \\
& =z^{-e_{F_{l}^{\prime}}^{\prime}-\cdots-e_{F_{j_{k-1}}^{\prime}}^{\prime}}+z^{-e_{F_{l}^{\prime}}^{\prime}-\cdots-e_{F_{j_{k+1}}^{\prime}}^{\prime}} \\
& =z^{e_{[l: k-1]}^{\prime}}+z^{e_{[l: k]}} \text {. }
\end{aligned}
$$

Note that the index shift in the last equality comes from the fact that $\mathrm{pa}\left(\mu_{F}(\underline{w})\right)$ has one less face of level $l$ than $\mathrm{pa}(\underline{w})$ as $F^{\prime}$ is of level $l+1$. So the claim follows for level $l$.

$i=l+1$ Let $F_{j_{r}}$ be the face of level $l+1$ in $\operatorname{Out}_{F}$ and $F_{j_{r+1}}$ the one in $\operatorname{In}_{F}$. Then we compute with notation as above

$$
\begin{aligned}
& \mu_{F^{\prime}}^{*}\left(z^{e_{[l: r]}}+z^{e_{[l: r+1]}}\right)=z^{-e_{F_{l+1}}-\cdots-e_{F_{j_{r}}}}\left(1+z^{e_{F}}\right)+z^{-e_{F_{l+1}}-\cdots-e_{F_{j_{r+1}}}} \\
& =z^{-e_{F_{l+1}}^{\prime}-\cdots-e_{F_{j_{r}}^{\prime}}^{\prime}}+z^{-e_{F_{l+1}^{\prime}}^{\prime}-\cdots-e_{F_{j_{r}}^{\prime}}^{\prime}-e_{F^{\prime}}^{\prime}}+z^{-e_{F_{l+1}^{\prime}}^{\prime}-\cdots-e_{F_{j_{r}}^{\prime}}^{\prime}-e_{F^{\prime}}^{\prime}-e_{F_{j_{r+1}}^{\prime}}^{\prime}} \\
& =z^{e_{[l+1: r]}^{\prime}}+z^{e_{[l+1: r+1]}^{\prime}}+z^{e_{[l+1: r+2]}} .
\end{aligned}
$$

As before the index shift occurs because $\mathrm{pa}\left(\mu_{F}(\underline{w})\right)$ has additionally the face $F^{\prime}$ of level $l+1$ in comparison to $\mathrm{pa}(\underline{w})$.

We can now prove the following theorem.

Theorem 6. Let $\underline{w}_{0}$ be an arbitrary reduced expression of $w_{0} \in S_{n}$. Then the superpotential expressed in the seed given by $\operatorname{pa}\left(\underline{w}_{0}\right)$ satisfies $\left.W\right|_{\underline{\mathcal{w}}_{0}}=W_{\mathcal{S}_{\underline{w}_{0}}}$. In particular,

$$
\left.W\right|_{\underline{\mathcal{X}}_{\underline{w}_{0}}}=\sum_{\mathbf{p} \in \mathcal{P}_{\underline{w}_{0}}} z^{e_{\mathbf{p}}}+\sum_{i \in[n-1], 0 \leq k \leq n_{i}} z^{e^{[i: k]} .}
$$

Proof. By Proposition 8 the claim is true for the seed $s_{0}$ with $\underline{w}_{0}=s_{1} s_{2} s_{1} \cdots s_{n-1} \cdots s_{2} s_{1}$. Now Lemmas 3 and 4 imply that the claim holds for all seeds that are related to $s_{0}$ by a finite sequence of mutations. As there are only finitely many reduced expressions for $w_{0}$ and they are all related by mutation as defined in Definition 7 the claim is true for all $\underline{w}_{0}$.

Remark 4. The theorem has the following consequence relating GP-paths with $\mathcal{X}$-cluster variables and $\vartheta$-functions. We have encoded GP-paths in two different ways: in terms of their crossing points in the pseudoline arrangement $c_{(i, j)}$ 's and in terms of the faces of the pseudoline arrangement they enclose $e_{F_{i}}$ 's. For a fixed orientation of $\mathrm{pa}\left(\underline{w}_{0}\right)$, say $\left(l_{i}, l_{i+1}\right)$, the detropicalization of the GP-data is a sum of corresponding $\mathcal{X}$-variables. In fact, by Theorem 6 this sum of $\mathcal{X}$-variables is the $\vartheta$-function associated to the frozen vertex $w_{(i, n)}$ expressed in the seed $s_{\underline{w}_{0}}$ : every GP-path $\mathbf{p}$ yields one summand $z^{e_{\mathbf{p}}}$ of $\vartheta_{(i, n)} \mid \mathcal{X}_{\underline{w}_{0}}$.

Corollary 2. For every reduced expression $\underline{w}_{0} \in S_{n}$ the following polyhedral objects coincide

(i) $\mathcal{S}_{\underline{w}_{0}}=\Xi_{\underline{w}_{0}}$,

(ii) $S_{\underline{w}_{0}}=\underline{\Xi}_{\underline{w}_{0}}$,

(iii) $\underline{\mathcal{S}}_{\underline{w}_{0}}(\lambda)=\Xi_{\underline{w}_{0}}(\lambda)$ for $\lambda \in \mathbb{R}^{n-1}$. 
Proof. The claim in (i) follows immediately from Theorem 6 by tropicalizing. Then (iii) follows by definition as we intersect both cones with the same collection of hyperplanes. To see (ii), recall from the proof of Proposition 8 that for the initial seed $s_{0}$ the $\vartheta$-functions $\vartheta_{(i, n)}$ correspond to GP-paths. Then the claim follows by Lemma 3 and the proof of Theorem 6.

\section{Applichtions of Theorem 4}

We have seen in the last two subsections how the cones and polytopes defined in $\S 3$ arise from a representation theoretic point of view and in the context of cluster varieties. The following theorem is the main combinatorial result of this section. We obtain it as an application of the unimodular equivalences in Theorem 4.

Theorem 7. Let $\underline{w}_{0}$ be an arbitrary reduced expression of $w_{0} \in S_{n}$. Then the following polyhedral objects are unimodularly equivalent

(i) $\mathcal{Q}_{\underline{w}_{0}} \cong \Xi_{\underline{w}_{0}}$ via $\Psi_{\underline{w}_{0}}$,

(ii) $Q_{\underline{w}_{0}} \cong \underline{\Xi}_{\underline{w}_{0}}$ via $\left.\Psi_{\underline{w}_{0}}\right|_{\mathbb{R}^{N}}$

(iii) $\underline{\mathcal{Q}}_{\underline{w}_{0}}(\lambda) \cong \Xi_{\underline{w}_{0}}(\lambda)$ for $\lambda \in \mathbb{R}^{n-1}$ via $\Psi_{\underline{w}_{0}}$.

Proof. Combine Theorem 4 with Theorem 5 and Corollary 2.

Remark 5. For the special case of the initial seed $s_{0}$ the theorem can also be proved by combining results of Magee and Littelmann. In [Lit98] Littelmann shows that the string polytope $\mathcal{Q}_{\underline{w}_{0}}(\lambda)$ for $\underline{w}_{0}=s_{1} s_{2} s_{1} \cdots s_{n-1} s_{n-2} \cdots s_{2} s_{1}$ is unimodularly equivalent to the Gelfand-Tsetlin polytope defined in [GT50]. Magee shows in [Mag15] that $\Xi_{s_{0}}$ (resp. $\Xi_{s_{0}}(\lambda)$ ) is unimodularly equivalent to the Gelfand-Tsetlin cone (resp. polytope). Combining both, one obtains Theorem 7 for $s_{0}$. In fact, to understand Magee's result was driving motivation behind this project.

By the construction of toric varieties associated to polytopes as in [CLS11, $§ 2.1$ and $\S 2.3$ ] and the toric degenerations of Caldero [Cal02] and Gross-Hacking-Keel-Kontsevich [GHKK18] we obtain the following corollary from Theorem 7 relating these toric varieties. It is the main result regarding toric degenerations of flag varieties in this section and an answer to Question 1 in the introduction.

Corollary 3. Let $\underline{w}_{0}$ be an arbitrary reduced expression of $w_{0} \in S_{n}$ and $\lambda \in \mathbb{Z}_{>0}^{n-1}$. We have an induced isomorphism of the following toric varieties that are degenerations (resp. normalizations of such) of $S L_{n} / B$

$$
X_{\mathcal{Q}_{\underline{w}_{0}}}(\lambda) \cong X_{\Xi_{\underline{w}_{0}}}(\lambda) .
$$

In order to achieve a similar result for Schubert varieties, we study the restriction of the superpotential in the following subsection.

6.1. Restricted Superpotential and Schubert varieties. Caldero's degeneration works more generally for Schubert varieties. As we have seen above, he uses the degeneration for the flag variety and by a quotient construction on the level of rings he obtains a family for the Schubert variety. For the cones, taking this quotient corresponds to setting certain variables to zero, or equivalently, restricting the defining GP-paths as in Definition 16. In a similar fashion we want to proceed with the superpotential. We show how the polytopes defining toric degenerations of Schubert varieties arise in the setting of [GHKK18].

Consider $w \in S_{n}$ with reduced expression $\underline{w}$ and extension $\underline{w}_{0}=\underline{w} s_{i_{\ell(w)+1}} \cdots s_{i_{N}}$. Recall that for a seed corresponding to $\underline{w}_{0}$ we have a basis $\left\{e_{F} \mid F\right.$ face of $\left.\operatorname{pa}\left(\underline{w}_{0}\right)\right\}$ for $N_{\underline{w}_{0}}$ and further 


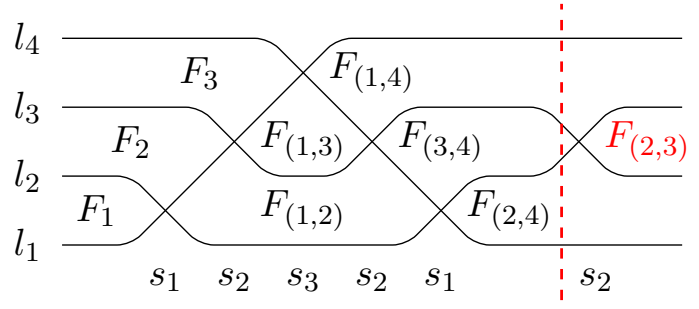

FiguRE 11. Restriction/Extension of a pseudoline arrangement.

$\mathbb{C}\left[\mathcal{X}_{w_{0}}\right]=\mathbb{C}\left[z^{ \pm e_{F}} \mid F\right.$ face of $\left.\mathrm{pa}\left(\underline{w}_{0}\right)\right]$. Then $\left\{e_{F} \mid F\right.$ face of $\left.\mathrm{pa}(\underline{w})\right\}$ generates a sublattice in $N_{\underline{w}_{0}}$, which we denote by $N_{\underline{w}}$ with dual lattice $M_{\underline{w}}$ a quotient of $M_{\underline{w}_{0}}$. We have the torus $\mathcal{X}_{\underline{w}}=T_{M_{\underline{w}}}=\operatorname{Spec}\left(\mathbb{C}\left[N_{\underline{w}}\right]\right)$ associated with $M_{\underline{w}}$ as in (2.1). In particular, $\mathbb{C}\left[\mathcal{X}_{\underline{w}}\right]=\mathbb{C}\left[z^{ \pm e_{F}} \mid\right.$ $F$ face of $\overline{\mathrm{pa}}(\underline{w})]$ and we have a restriction morphism between the Laurent polynomial rings

$$
\operatorname{res}_{\underline{w}}: \mathbb{C}\left[\mathcal{X}_{\underline{w}_{0}}\right] \rightarrow \mathbb{C}\left[\mathcal{X}_{\underline{w}}\right],\left.\quad f \mapsto f\right|_{\mathcal{X}_{\underline{w}}} .
$$

We are interested in the restrictions to $\mathcal{X}_{\underline{w}}$ of the superpotential $\left.W\right|_{\underline{\mathcal{X}}_{0}}$ and the detropicalization $W_{\mathcal{S}_{\underline{w}_{0}}}$ of $\mathcal{S}_{\underline{w}_{0}}$ (they are equal by Theorem 6). We want to show that they coincide with the detropicalization of $\mathcal{S}_{\underline{w}}$. In analogy with Definiton 21 for $w_{0}$ we consider for arbitrary $w$ the following polyhedral objects.

Definition 25. For $w \in S_{n}$ with reduced expression $\underline{w}$ and an extension $\underline{w}_{0}=\underline{w} s_{i_{\ell(w)+1}} \cdots s_{i_{N}}$ polyhedral objects by tropicalizing the restriction of a sum of $\vartheta$-functions resp. the superpotential:

$$
\begin{aligned}
& \operatorname{res}_{\underline{w}}\left(\Xi_{\underline{w}_{0}}\right):=\left\{\mathbf{x} \in \mathbb{R}^{\ell(w)+n-1} \mid \operatorname{res}_{\underline{w}}\left(\left.W\right|_{\mathcal{X}_{\underline{w}_{0}}}\right)^{\operatorname{trop}}(\mathbf{x}) \geq 0\right\}, \\
& \operatorname{res}_{\underline{w}}\left(\Xi_{\underline{w}_{0}}\right):=\left\{\mathbf{x} \in \mathbb{R}^{\ell(w)} \mid \operatorname{res}_{\underline{w}}\left(\sum_{i=1}^{n-1} \vartheta_{(i, n)} \mid \mathcal{X}_{\underline{w}_{0}}\right)^{\operatorname{trop}}(\mathbf{x}) \geq 0\right\}, \\
& \operatorname{res}_{\underline{w}}\left(\Xi_{\underline{w}_{0}}(\lambda)\right):=\operatorname{res}_{\underline{w}}\left(\Xi_{\underline{w}_{0}}\right) \cap \tau_{\underline{w}}^{-1}(\lambda) \text { for } \lambda \in \mathbb{R}^{n-1} .
\end{aligned}
$$

Example 12. Consider $\underline{w}=s_{1} s_{2} s_{3} s_{2} s_{1} \in S_{4}$ with extension $\underline{w}_{0}=\underline{w} s_{2}$. We compute the

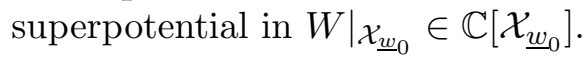

$$
\begin{aligned}
\left.W\right|_{\underline{\mathcal{w}}_{0}} & =\left(z^{-e_{3}}+z^{-e_{3}-e_{(1,4)}}\right)+\left(z^{-e_{2}}+z^{-e_{2}-e_{(1,3)}}+z^{-e_{2}-e_{(1,3)}-e_{(3,4)}}\right) \\
& +\left(z^{-e_{1}}+z^{-e_{1}-e_{(1,2)}}+z^{-e_{1}-e_{(1,2)}-e_{(2,4)}}\right)+\left(z^{-e_{(2,4)}}+z^{-e_{(2,4)}-e_{(3,4)}}\right)+\left(z^{-e_{2,3}}\right) \\
& +\left(z^{-e_{(1,4)}}+z^{-e_{(1,4)}-e_{(1,3)}}+z^{-e_{(1,4)}-e_{(1,3)}-e_{(3,4)}}+z^{-e_{(1,4)}-e_{(1,3)}-e_{(3,4)}-e_{(1,2)}}\right) .
\end{aligned}
$$

From Figure 11 we see that $F_{(2,3)}$ is a face of $\mathrm{pa}\left(\underline{w}_{0}\right)$, but not of $\mathrm{pa}(\underline{w})$. Hence,

$$
\begin{aligned}
\operatorname{res}_{\underline{w}}\left(\left.W\right|_{\underline{x}_{\underline{w}}}\right) & =\left(z^{-e_{3}}+z^{-e_{3}-e_{(1,4)}}\right)+\left(z^{-e_{2}}+z^{-e_{2}-e_{(1,3)}}+z^{-e_{2}-e_{(1,3)}-e_{(3,4)}}\right) \\
& +\left(z^{-e_{1}}+z^{-e_{1}-e_{(1,2)}}+z^{-e_{1}-e_{(1,2)}-e_{(2,4)}}\right)+\left(z^{-e_{(2,4)}}+z^{-e_{(2,4)}-e_{(3,4)}}\right) \\
& +\left(z^{-e_{(1,4)}}+z^{-e_{(1,4)}-e_{(1,3)}}+z^{-e_{(1,4)}-e_{(1,3)}-e_{(3,4)}}+z^{-e_{(1,4)}-e_{(1,3)}-e_{(3,4)}-e_{(1,2)}}\right) .
\end{aligned}
$$

Proposition 9. Let $w \in S_{n}$ and consider a reduced expression $\underline{w}$ with an extension to $\underline{w}_{0}=\underline{w} s_{i_{\ell(w)+1}} \cdots s_{i_{N}}$. Then

$$
\operatorname{res}_{\underline{w}}\left(\left.W\right|_{\mathcal{X}_{\underline{w}_{0}}}\right)=W_{\mathcal{S}_{\underline{w}}} .
$$


Proof. Recall the restriction of GP-paths defined in Definition 16. By Propositions 4 and 6 we have seen $\operatorname{res}_{\underline{w}}\left(\mathcal{P}_{\underline{w}_{0}}\right)=\mathcal{P}_{\underline{w}}$. To avoid confusion we denote as before for $i \in[n-1]$ by $n_{i}^{\underline{w}}:=\#\left\{j \mid s_{i_{j}}=s_{i}\right.$ in $\left.\underline{w}\right\}$ and $n_{i}^{\underline{w}}:=\#\left\{j \mid s_{i_{j}}=s_{i}\right.$ in $\left.\underline{w}_{0}\right\}$. Using Theorem 6 we compute

$$
\begin{aligned}
\operatorname{res}_{\underline{w}}\left(\left.W\right|_{\mathcal{X}_{\underline{w}}}\right) & =\sum_{\mathbf{p} \in \mathcal{P}_{\underline{w}}} z^{e_{\mathbf{p}}} \mid \mathcal{X}_{\underline{w}}+\sum_{i \in[n-1], 0 \leq k \leq n i \underline{w}_{0}} z^{e^{[i: k]} \mid \mathcal{X}_{\underline{w}}} \\
& =\sum_{\mathbf{p} \in \operatorname{res}_{\underline{w}}\left(\mathcal{P}_{\underline{w}_{0}}\right)} z^{e_{\mathbf{p}}}+\sum_{i \in[n-1], 0 \leq k \leq n} z^{e[i: k]}=W_{\mathcal{S}_{\underline{i}}} .
\end{aligned}
$$

Theorem 8. Let $w \in S_{n}$ and consider a reduced expression $\underline{w}$ with an extension to $\underline{w}_{0}=$ $\underline{w} s_{i_{\ell(w)+1}} \cdots s_{i_{N}}$. Then the following polyhedral objects are unimodularly equivalent

(i) $\mathcal{Q}_{\underline{w}} \cong \operatorname{res}_{\underline{w}}\left(\Xi_{\underline{w}_{0}}\right)$ via $\Psi_{\underline{w}}$,

(ii) $Q_{\underline{w}} \cong \operatorname{res}_{\underline{w}}\left(\Xi_{\underline{w}_{0}}\right)$ via $\left.\Psi_{\underline{w}}\right|_{\mathbb{R}^{\ell(w)}}$,

(iii) $\mathcal{Q}_{\underline{w}}(\lambda) \cong \operatorname{res}_{\underline{w}}\left(\Xi_{\underline{w}_{0}}(\lambda)\right)$ for $\lambda \in \mathbb{R}^{n-1}$ via $\Psi_{\underline{w}}$.

Proof. For (i) combine Proposition 9 with Theorem 4 and Theorem 5, which directly implies (iii). To see (ii), recall that by Lemma 3 and the proof of Proposition 8 we have

$$
\sum_{\mathbf{p} \in \mathcal{P}_{\underline{w}_{0}}} z^{e^{\mathbf{p}}}=\sum_{i \in[n-1]} \vartheta_{(i, n)} \mid \mathcal{X}_{\underline{w}_{0}} .
$$

By the proof of Proposition 9 the same equality when replacing $\underline{w}_{0}$ by $\underline{w}$. Then the claim follows by Theorem 4 and Theorem 5 .

Before stating the following corollary relating the toric degenerations of Schubert varieties by Caldero [Cal02] to the toric degenerations of flag varieties by Gross-Hacking-Keel-Kontsevich [GHKK18] we briefly remind you about the Orbit-Cone-Correspondence for toric varieties (see [CLS11, §3.2]). For a (full-dimensional) polytope $P \subset \mathbb{R}^{n}$ denote by $\Sigma_{P} \subset \mathbb{R}^{n}$ its normal fan (see [CLS11, Remark 2.3.3]). Every cone $\sigma \in \Sigma_{P}$ corresponds to a torus orbits in $X_{\Sigma_{P}}$ of dimension $n-\operatorname{dim} \sigma$ ( [CLS11, Theorem 3.2.6]). The closure of each torus orbit is a toric variety. For a face $Q$ of $P$ let $\sigma_{Q} \in \Sigma_{P}$ be the cone in $\Sigma_{P}$ spanned by the normal vectors of all facets of $P$ containing $Q$. Then by [CLS11, Proposition 3.2.9] the toric variety $X_{Q}$ is isomorphic to the closure of the torus orbit corresponding to the cone $\sigma_{Q} \in \Sigma_{P}$.

Consider an arbitrary $w \in S_{n}$ with a reduced expression $\underline{w}$ and $\underline{w}_{0}=\underline{w} s_{i_{\ell(w)+1}} \cdots s_{i_{N}}$ an extension. For every $\lambda \in \Lambda^{++}$recall that the toric variety $X_{\mathcal{Q}_{\underline{w}}(\lambda)}$ is (the normalization of) a toric degeneration of $X_{w}$ by [Cal02]. Similarly, $X_{\Xi_{\underline{w}_{0}}(\lambda)}$ is a flat degeneration of $S L_{n} / B$ by [GHKK18]. We can now formulate the geometric version of our main result on toric degenerations of Schubert varieties.

Corollary 4. The toric variety $X_{\mathcal{Q}_{\underline{w}}(\lambda)}$ is isomorphic to a subvariety of $X_{\Xi_{\underline{w}_{0}}(\lambda)}$. More precisely, we have

$$
X_{\mathcal{Q}_{\underline{w}}(\lambda)} \cong X_{\operatorname{res}_{\underline{w}}\left(\Xi_{\underline{w}_{0}}(\lambda)\right)},
$$

where $X_{\operatorname{res}_{\underline{w}}\left(\Xi_{\underline{w}_{0}}(\lambda)\right)}$ is the closure of the torus orbit corresponding to the cone $\sigma_{\operatorname{res}_{\underline{w}}\left(\Xi_{\underline{w}_{0}}(\lambda)\right)} \in$ $\Sigma_{\Xi_{\underline{w}_{0}}(\lambda)}$.

Proof. By definition $\operatorname{res}_{\underline{w}}\left(\Xi_{\underline{w}_{0}}(\lambda)\right)$ is a union of faces of $\Xi_{\underline{w}_{0}}(\lambda)$. Theorem 8(iii) implies in particular, that $\operatorname{res}_{\underline{w}}\left(\Xi_{\underline{w}_{0}}(\lambda)\right)$ is a polytope itself, hence a face of $\Xi_{\underline{w}_{0}}(\lambda)$. Further, the 
unimodular equivalence $Q_{\underline{w}}(\lambda) \cong \operatorname{res}_{\underline{w}}\left(\Xi_{\underline{w}_{0}}(\lambda)\right)$ induces an isomorphism of toric varieties $X_{Q_{\underline{w}}(\lambda)} \cong X_{\operatorname{res}_{\underline{w}}\left(\Xi_{\underline{w}_{0}}(\lambda)\right)}$. Then the Corollary follows by [CLS11, Proposition 3.2.9].

6.2. Restriction vs. superpotential for double Bruhat cells. We conclude with an example that shows how $\operatorname{res}_{\underline{w}}\left(\left.W\right|_{\mathcal{X}_{\underline{w}}}\right)$ is essentially different from a function one would obtain from applying Algorithm 1 to the quiver $Q_{\underline{w}}$

Example 13. Let $s=s_{\underline{w}}$ be the seed of the reduced expression $\underline{w}=s_{1} s_{2} s_{3} s_{2} s_{1} \in S_{4}$ as in Figure 11. The corresponding quiver is pictured in Figure 12. We apply Algorithm 1 and compute optimized seeds for all frozen vertices in $Q_{\underline{w}}$. As $w_{3}$ and $w_{(2,4)}$ are sinks in $Q_{\underline{w}}$ we set $\left.\vartheta_{3}\right|_{\mathcal{X}_{s}}=z^{-e_{3}}$ and $\vartheta_{(2,4)} \mid \mathcal{X}_{s}=z^{-e_{(2,4)}}$, where $\left\{e_{1}, e_{2}, e_{3}, e_{(1,2)}, e_{(1,3)}, e_{(1,4)}, e_{(2,4)}, e_{(3,4)}\right\}$ is the lattice basis associated to $s$.
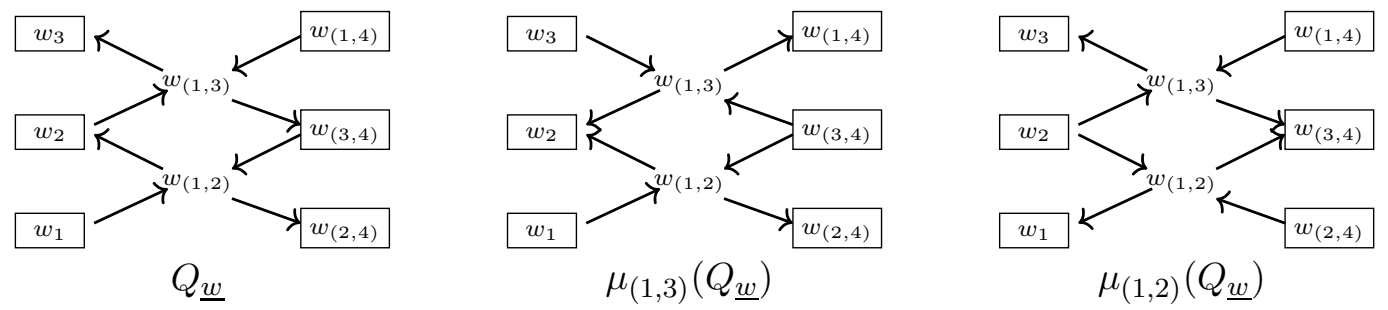

Figure 12. The quivers $Q_{\underline{w}}, \mu_{(1,3)}\left(Q_{\underline{w}}\right)$ and $\mu_{(1,2)}\left(Q_{\underline{w}}\right)$ for $\underline{w}=s_{1} s_{2} s_{3} s_{2} s_{1}$.

The boxes denote frozen variables.

For the other variables we have to find a mutation sequence to an optimized seed. Mutation at $w_{(1,3)}$ (resp. $\left.w_{(1,2)}\right)$ yields the quiver $\mu_{(1,3)}\left(Q_{\underline{w}}\right)\left(\operatorname{resp} . \mu_{(1,2)}\left(Q_{\underline{w}}\right)\right)$ in Figure 12. The seed $\mu_{(1,3)}(s)$ is optimized for $w_{(1,4)}$ and $w_{2}$, so $\left.\vartheta_{(1,4)}\right|_{\mathcal{X}_{\left.\mu_{(1,3)} \underline{w}\right)}}=z^{-e_{(1,4)}^{\prime}}$ and $\left.\vartheta_{2}\right|_{\mathcal{X}_{\left.\mu_{(1,3)} \underline{w}\right)}}=z^{-e_{2}^{\prime}}$. In $\mathcal{X}_{\underline{w}}$ we obtain $\vartheta_{(1,4)} \mid \mathcal{X}_{s}=z^{-e_{(1,4)}}+z^{-e_{(1,4)}-e_{(1,3)}}$ and $\left.\vartheta_{2}\right|_{\mathcal{X}_{s}}=z^{-e_{2}}+z^{-e_{2}-e_{(1,3)}}$. Proceeding analogously with $\mu_{(1,2)}(s)$, optimized for $w_{(3,4)}$ and $w_{1}$, we obtain a function on $\mathcal{X}_{\underline{w}}$

$$
\begin{aligned}
F & :=\left(z^{-e_{3}}\right)+\left(z^{-e_{2}}+z^{-e_{2}-e_{(1,3)}}\right)+\left(z^{-e_{1}}+z^{-e_{1}-e_{(1,2)}}\right)+\left(z^{-e_{(2,4)}}\right) \\
& +\left(z^{-e_{(3,4)}}+z^{-e_{(3,4)}-e_{(1,2)}}\right)+\left(z^{-e_{(1,4)}}+z^{-e_{(1,4)}-e_{(1,3)}}\right) .
\end{aligned}
$$

Comparing to Example 12 where $\underline{w}_{0}=\underline{w} s_{2}$ we observe that $F \neq \operatorname{res}_{\underline{w}}\left(\left.W\right|_{\mathcal{X}_{\underline{w}_{0}}}\right)$. Tropicalizing $\operatorname{res}_{\underline{w}}\left(\left.W\right|_{\mathcal{X}_{\underline{w}_{0}}}\right)$ we get the following set of inequalities defining the cone $\mathcal{S}_{\underline{w}} \subset \mathbb{R}^{8}$

$$
\begin{aligned}
& -x_{3} \geq 0, \quad-x_{3}-x_{(1,4)} \geq 0, \\
& -x_{2} \geq 0, \quad-x_{2}-x_{(1,3)} \geq 0, \quad-x_{2}-x_{(1,3)}-x_{(3,4)} \geq 0 \\
& -x_{1} \geq 0, \quad-x_{1}-x_{(1,2)} \geq 0, \quad-x_{1}-x_{(1,2)}-x_{(2,4)} \geq 0, \\
& -x_{(2,4)} \geq 0, \quad-x_{(2,4)}-x_{(3,4)} \geq 0, \quad-x_{(1,4)}-x_{(1,3)}-x_{(3,4)} \geq 0, \\
& -x_{(1,4)} \geq 0, \quad-x_{(1,4)}-x_{(1,3)} \geq 0, \quad-x_{(1,4)}-x_{(1,3)}-x_{(3,4)}-x_{(1,2)} \geq 0 .
\end{aligned}
$$


From $F^{\text {trop }}$ we get inequalities defining a cone $\mathcal{D}_{F} \subset \mathbb{R}^{8}$ :

$$
\begin{array}{rlr}
-x_{3} & \geq 0, & \\
-x_{2} & \geq 0, & -x_{2}-x_{(1,3)} \geq 0, \\
-x_{1} & \geq 0, & -x_{1}-x_{(1,2)} \geq 0, \\
-x_{(2,4)} & \geq 0, & \\
-x_{(3,4)} & \geq 0, & -x_{(3,4)}-x_{(1,2)} \geq 0, \\
-x_{(1,4)} & \geq 0, & -x_{(1,4)}-x_{(1,3)} \geq 0 .
\end{array}
$$

Observe that $\mathcal{D}_{F} \subset \mathcal{S}_{\underline{w}}$. We compute the polytopes $\mathcal{S}_{\underline{w}}(\lambda)$ and $\mathcal{D}_{F} \cap \tau_{\underline{w}}^{-1}(\lambda)$ for $\lambda=(1,1,1)$ and their lattice points using polymake [GJ00]. The outcome is

$$
\left|\mathcal{S}_{\underline{w}}(\lambda) \cap \mathbb{Z}^{8}\right|=49=\operatorname{dim}_{\mathbb{C}} H^{0}\left(X_{w}, L_{\lambda}\right)>\left|\mathcal{D}_{F} \cap \tau_{\underline{w}}^{-1}(\lambda) \cap \mathbb{Z}^{8}\right|=30 .
$$

In particular, the toric variety $X_{\mathcal{D}_{F} \cap \tau_{\underline{w}}^{-1}(\lambda)}$ can not be a flat degeneration of the Schubert variety $X_{w}$. However, this observation is not too surprising from a geometric point of view, as the restricted superpotential and the function $F$ correspond to different partial compactifications of the $\mathcal{A}$-cluster variety $G^{e, w}$ associated with $\mathcal{Y}\left(s_{w}\right)$. When considering the restricted superpotential, the cluster variety we are dealing with is $\bar{G}^{e, w_{0}}$ and its compactification $\bar{G}^{e, w_{0}}$ with boundary divisors

$$
\left\{\bar{p}_{1}=0\right\},\left\{\bar{p}_{12}=0\right\},\left\{\bar{p}_{123}=0\right\},\left\{\bar{p}_{4}=0\right\},\left\{\bar{p}_{34}=0\right\},\left\{\bar{p}_{234}=0\right\} .
$$

Recall that $G^{e, w_{0}}$ is $S L_{4} / U$ up to codimension 2. The Schubert variety of our interest is $X_{w}$ with $s_{1} s_{2} s_{3} s_{2} s_{1}=w$. It is given by $\left\{\bar{p}_{34}=0\right\}$ as a subvariety $S L_{4} / B$. Note that in fact, whenever we have a reduced expression $\underline{w}$ and an extension $\underline{w}_{0}=\underline{w} s_{i_{\ell(w)+1}} \cdots s_{i_{N}}$, then the Plücker coordinates that appear as $\mathcal{A}$-cluster variables for faces of $\mathrm{pa}\left(\underline{w}_{0}\right)$ that are not faces of $\mathrm{pa}(\underline{w})$ vanish identically on $X_{w}$. When restricting the superpotential, we consider the divisor of $\bar{G}^{e, w_{0}}$ (resp. $S L_{4} / U$ ) given by $\left\{\bar{p}_{34}=0\right\}$, which is closely related to $X_{w}$.

The function $F$ on the other hand corresponds to the $\mathcal{A}$-cluster variety $G^{e, w}$ and its partial compactification $\bar{G}^{e, w}$ with boundary divisors

$$
\left\{\bar{p}_{1}=0\right\},\left\{\bar{p}_{12}=0\right\},\left\{\bar{p}_{123}=0\right\},\left\{\bar{p}_{4}=0\right\},\left\{\bar{p}_{24}=0\right\},\left\{\bar{p}_{234}=0\right\} .
$$

In this case, the defining equation for $X_{w}$ in $S L_{4} / B$ is not part of the boundary, so there is no reason to expect information for the Schubert variety from the potential $F$ encoding this boundary.

\section{REFERENCES}

[And13] Dave Anderson. Okounkov bodies and toric degenerations. Mathematische Annalen, 356(3):11831202, 2013.

[BFMn18] Lara Bossinger, Bosco Frías-Medina, Timothy Magee, and Alfredo Nájera Chávez. Toric degenerations of cluster varieties and cluster duality. arXiv preprint arXiv:1809.08369, 2018.

[BFZ96] Arkady Berenstein, Sergey Fomin, and Andrei Zelevinsky. Parametrizations of canonical bases and totally positive matrices. Adv. Math., 122(1):49-149, 1996.

[BFZ05] Arkady Berenstein, Sergey Fomin, and Andrei Zelevinsky. Cluster algebras. III. Upper bounds and double Bruhat cells. Duke Math. J., 126(1):1-52, 2005.

[BZ01] Arkady Berenstein and Andrei Zelevinsky. Tensor product multiplicities, canonical bases and totally positive varieties. Inventiones mathematicae, 143(1):77-128, 2001.

[Cal02] Philippe Caldero. Toric degenerations of Schubert varieties. Transformation groups, 7(1):51-60, 2002.

[CLS11] David A. Cox, John B. Little, and Henry K. Schenck. Toric varieties. American Mathematical Soc., 2011. 
[FFL17] Xin Fang, Ghislain Fourier, and Peter Littelmann. Essential bases and toric degenerations arising from birational sequences. Adv. Math., 312:107-149, 2017.

[FG06] V. V. Fock and A. B. Goncharov. Cluster $\mathcal{X}$-varieties, amalgamation, and Poisson-Lie groups. In Algebraic geometry and number theory, volume 253 of Progr. Math., pages 27-68. Birkhäuser Boston, Boston, MA, 2006.

[FWZ16] Sergey Fomin, Lauren Williams, and Andrei Zelevinsky. Introduction to cluster algebras. chapters 1-3. arXiv preprint arXiv:1608.05735, 2016.

[FZ02] Sergey Fomin and Andrei Zelevinsky. Cluster algebras. I. Foundations. J. Amer. Math. Soc., 15(2):497-529, 2002.

[GHK15] Mark Gross, Paul Hacking, and Sean Keel. Birational geometry of cluster algebras. Algebr. Geom., 2(2):137-175, 2015.

[GHKK18] Mark Gross, Paul Hacking, Sean Keel, and Maxim Kontsevich. Canonical bases for cluster algebras. J. Amer. Math. Soc., 31(2):497-608, 2018.

[GJ00] Ewgenij Gawrilow and Michael Joswig. Polymake: a framework for analyzing convex polytopes. In Polytopes-combinatorics and computation, pages 43-73. Springer, 2000.

[GKS16] Volker Genz, Gleb Koshevoy, and Bea Schumann. Combinatorics of canonical bases revisited: Type A. arXiv preprint arXiv:1611.03465, 2016.

[GKS17] Volker Genz, Gleb Koshevoy, and Bea Schumann. Polyhedral parametrizations of canonical bases \& cluster duality. arXiv preprint arXiv:1711.07176, 2017.

[GL96] Nicolae Gonciulea and Venkatramani Lakshmibai. Degenerations of flag and Schubert varieties to toric varieties. Transformation Groups, 1(3):215-248, 1996.

[GLS13] Christof Geiss, Bernard Leclerc, and Jan Schröer. Cluster algebras in algebraic Lie theory. Transform. Groups, 18(1):149-178, 2013.

[GP00] Oleg Gleizer and Alexander Postnikov. Littlewood-Richardson coefficients via Yang-Baxter equation. International Mathematics Research Notices, 2000(14):741-774, 2000.

[GS15] Alexander Goncharov and Linhui Shen. Geometry of canonical bases and mirror symmetry. Invent. Math., 202(2):487-633, 2015.

[GT50] I. M. Gelfand and M. L. Tsetlin. Finite-dimensional representations of the group of unimodular matrices. Doklady Akad. Nauk SSSR (N.S.), 71:825-828, 1950.

[HL16] Takayuki Hibi and Nan Li. Unimodular equivalence of order and chain polytopes. Math. Scand., 118(1):5-12, 2016.

[Kav15] Kiumars Kaveh. Crystal bases and Newton-Okounkov bodies. Duke Mathematical Journal, 164(13):2461-2506, 2015.

[Kel] Bernhard Keller. Quiver mutation in javascript and java. Available at https://webusers.imj-prg.fr/ bernhard.keller/quivermutation/.

[KK12] Kiumars Kaveh and A. G. Khovanskii. Newton-Okounkov bodies, semigroups of integral points, graded algebras and intersection theory. Ann. of Math. (2), 176(2):925-978, 2012.

[KM16] Kiumars Kaveh and Christopher Manon. Khovanskii bases, higher rank valuations and tropical geometry. arXiv preprint arXiv:1610.00298, 2016.

[Lec16] B. Leclerc. Cluster structures on strata of flag varieties. Adv. Math., 300:190-228, 2016.

[Lit98] Peter Littelmann. Cones, crystals, and patterns. Transformation groups, 3(2):145-179, 1998.

[Mag15] Timothy Magee. Fock-Goncharov conjecture and polyhedral cones for $U \subset S L_{n}$ and base affine space $S L_{n} / U$. arXiv preprint arXiv:1502.03769, 2015.

[MS15] Diane Maclagan and Bernd Sturmfels. Introduction to tropical geometry, volume 161 of Graduate Studies in Mathematics. American Mathematical Society, Providence, RI, 2015.

[RW17] Konstanze Rietsch and Lauren Williams. Newton-Okounkov bodies, cluster duality, and mirror symmetry for Grassmannians. arXiv preprint arXiv:1712.00447, 2017.

[Sco06] Joshua S. Scott. Grassmannians and cluster algebras. Proc. London Math. Soc. (3), 92(2):345-380, 2006.

L. Bossinger: Instituto de Matemáticas unam Unidad Oaxaca, León 2, altos, Oaxaca de Juárez, Centro Histórico, 68000 OAxaca, Mexico

E-mail address: lara@im.unam.mx

G. Fourier: RWTH Aachen University, Pontdriesch 10-16, 52062 Aachen

E-mail address: fourier@mathb.rwth-aachen.de 